\title{
Rebooting Psychotherapy Research and Practice to Reduce the Burden of Mental IIIness
}

Perspectives on Psychological Science 6(I) $21-37$

(C) The Author(s) 2011

Reprints and permission:

sagepub.com/journalsPermissions.nav DOI: $10.1177 / 1745691610393527$

http://pps.sagepub.com

(S)AGE

\author{
Alan E. Kazdin and Stacey L. Blase \\ Department of Psychology, Yale University, New Haven, CT
}

\begin{abstract}
Psychological interventions to treat mental health issues have developed remarkably in the past few decades. Yet this progress often neglects a central goal-namely, to reduce the burden of mental illness and related conditions. The need for psychological services is enormous, and only a small proportion of individuals in need actually receive treatment. Individual psychotherapy, the dominant model of treatment delivery, is not likely to be able to meet this need. Despite advances, mental health professionals are not likely to reduce the prevalence, incidence, and burden of mental illness without a major shift in intervention research and clinical practice. A portfolio of models of delivery will be needed. We illustrate various models of delivery to convey opportunities provided by technology, special settings and nontraditional service providers, self-help interventions, and the media. Decreasing the burden of mental illness also will depend on integrating prevention and treatment, developing assessment and a national database for monitoring mental illness and its burdens, considering contextual issues that influence delivery of treatment, and addressing potential tensions within the mental health professions. Finally, opportunities for multidisciplinary collaborations are discussed as key considerations for reducing the burden of mental illness.
\end{abstract}

\section{Keywords}

psychological interventions, reducing the burden of mental illness

Psychological interventions to treat clinical dysfunction have advanced remarkably in the past few decades. The progress is evident in many ways. First, the quantity of controlled treatment outcome studies has proliferated. Empirical studies of therapy for children, adolescents, and adults number well into the thousands. Many journals feature therapy outcome research as their primary thrust so the flow of research continues. Second, the quality of research has continued to improve as well. The use of randomized controlled trials (RCTs) is recognized as the fundamental design, but many other methodological features (e.g., the use of treatment manuals, assessment of clinical significance of change, evaluation of follow-up) have set the bar high for treatment outcome studies. Third, and perhaps most salient, has been the delineation of evidence-based treatments (EBTs; i.e., interventions with strong evidence on their behalf). EBTs are available for many psychological dysfunctions for children, adolescents, and adults (e.g., Nathan \& Gorman, 2007; Weisz \& Kazdin, 2010). EBTs continue to emerge and reflect palpable progress from scientific research.

The remarkable progress has left in the background a key issue that is a major impetus for developing psychological interventions - namely, the goal of decreasing rates of mental illness and improving psychosocial functioning on a large scale (i.e., in society). Psychological treatments have many purposes, but key among them is to alleviate mental illnesses and related sources of dysfunction. A central thesis of this article is that, despite advances in research, mental health professionals may have little success in decreasing the prevalence and incidence of mental illness without a major shift and expansion of intervention research and clinical practice. The article focuses on models of treatment delivery and what is needed to reduce the burden. By burden, we refer to the personal, social, and monetary costs associated with impairment. Within the term mental illness, we include psychiatric disorders and also social, cognitive, emotional, and behavioral sources of impairment or disability.

\section{Corresponding Author:}

Alan E. Kazdin, Department of Psychology, 2 Hillhouse Avenue, Yale University, New Haven, CT 06520-8205

E-mail: alan.kazdin@yale.edu 
We begin by highlighting the burden and cost of mental illness and associated psychological sources of dysfunction. We then discuss why advances in current treatment are not likely to have broad impact and reach most people in need. We highlight individual psychotherapy as a point of departure because it serves as the dominant model of treatment delivery and is emphasized in treatment research, clinical practice, and training in the mental health professions. By model of delivery, we refer to multiple characteristics of how an intervention is administered, by whom, under what conditions, and in what contexts. Psychotherapy as a model usually is delivered to one person at a time (or couple, family, or in a small group) by a trained mental health professional at a health care or mental health service facility or private office. Although there are many variations of therapy (techniques), the model of delivery is more narrowly restricted among them. Yet emphasis on this one delivery model leaves enormous gaps that must be addressed to reduce the burden of mental illness.

In addition, this article highlights research on the burden of mental illness and the current treatment model as steps toward elaborating changes that are needed for providing treatment. We illustrate several models of delivery that expand on the model of individual psychotherapy. The burden of mental illness can be reduced by expanding models of delivery. At the same time, reducing the burden raises other considerations including the integration of prevention and treatment, the need for improved assessment to monitor psychological dysfunction nationwide, contextual factors that influence health care, professional tensions within clinical psychology, and important opportunities for collaborating with other disciplines.

\section{Reducing the Burden of Mental Illness and Related Conditions}

The challenge for psychological interventions is to help reduce the burden of mental illness and related conditions both at the personal and societal level. ${ }^{1}$ Four interrelated considerations convey why diverse treatment delivery models are needed.

\section{Rate of Mental IIIness}

Consider the rate of psychological dysfunction. Not all sources of psychological impairment are codified by current classification systems of psychiatric disorders (Diagnostic and Statistical Manual of Mental Disorders, DSM-IV-TR; American Psychiatric Association, 2000; International Classification of Diseases, ICD-10; World Health Organization, 2007). Impairment can result from many sources (e.g., stress, relationship problems) beyond those included in diagnostic systems. Also, many disorders are on a spectrum indicating continuity of dysfunction. For example, several symptoms of depression are required to meet criteria for a DSM diagnosis. However, just failing to meet the criteria (e.g., by a symptom or duration requirement) is still associated with dysfunction or impairment, commensurate with the discrepancy from meeting the criteria. Subthreshold dysfunction leads to prevalence rates that underestimate the burden of dysfunction. Nevertheless, data on psychiatric disorders, albeit conservative, are instructive in illustrating the scope of psychological dysfunction.

Lifetime prevalence rates reveal that mental illness (meeting criteria for a psychiatric disorder) is relatively common, not only within the United States, but also within many countries worldwide. A series of recent surveys from the World Health Organization assessing the global burden of mental illness found a lifetime $D S M-I V$ disorder prevalence within its 17 participating countries of $12.0 \%$ to $47.4 \%$, with the highest lifetime prevalence estimate in the United States (Kessler et al., 2009). The same surveys reported the United States to have the highest 12-month $D S M$ - $I V$ disorder prevalence, with a range of $6.0 \%$ to $27.0 \%$ for all 17 countries. Summarizing the U.S. data only, approximately $50 \%$ of the population meets criteria for one or more psychiatric disorders in their lifetimes, and approximately $25 \%$ of the population meets criteria in any given year (Kessler \& Wang, 2008).

The rates of dysfunction vary as a function of culture, ethnicity, immigrant status within a given ethnicity, geographical location, and socioeconomic status, among other factors (e.g., Alegría et al., 2008). These variations and differences are important in developing interventions. For present purposes, we merely wish to convey that psychiatric disorders are prevalent. The estimates are likely to be conservative because they have required meeting diagnostic criteria and exclude those who do not quite meet criteria but are close enough to make the distinction of meeting or not meeting the criteria minor.

\section{Cost of Mental IIIness}

The costs of mental illness are high. Although there is no single, agreed upon figure or set of figures of those costs, welldocumented examples in specific problem domains convey the point. For example, alcoholism and substance abuse, which affects more than 20 million Americans and is the most prevalent mental disorder in the United States, costs approximately $\$ 500$ billion annually (Jason \& Ferrari, 2010). The main costs include medical and criminal justice costs, accidents, and loss of earnings. For anxiety disorders, annual health-care expenditures in the United States are approximately $\$ 42$ billion (Greenberg et al., 1999). The costs encompass health-care utilization, including medical and psychiatric treatment, and decreased work productivity (see also H. Harwood, Fountain, \& Livermore, 1998).

Reductions in annual earnings also are associated with the diagnosis of a mental illness. Individuals diagnosed with a $D S M-I V$ mental disorder earn, on average, approximately $\$ 16,000$ less than their control counterparts annually. This results in a total reduction of $\$ 193.2$ billion in personal earnings nationally in 1 year (Kessler et al., 2008). A single episode of major depressive disorder is associated with an average of more than 5 weeks of lost productivity per worker, resulting in an annual capital loss of $\$ 36$ billion to employers (Kessler et al., 2006).

Cost extends beyond the fiscal burden; personal impairment and subjective experience are not trivial. In one series of 
national interviews, participants reported the number of days in the past month in which they were unable to perform their usual daily tasks due to problems because of physical or emotional health. Mental disorders were associated with more than half of the role-disability days, and depression had one of the largest effects on disability of all conditions (Merikangas et al., 2007). Mental disorders are more impairing than common chronic medical disorders, with particularly greater impairment in the domains of home, social, and close relationship functioning (Druss et al., 2009). These findings document the importance of the loss of productivity due to mental illness and also reflect the widespread interpersonal difficulties. These psychosocial sources of dysfunction that likewise lead to impairment, suffering, and costs to individuals as well as to society are more difficult to capture on a national level, but they are an important target for interventions to reduce the burden of mental illness.

\section{People in Need of Services}

A vital aspect to reducing the burden of mental illness is the availability of interventions for those most in need of services. Recent years have seen an increase in the rate of people in need receiving treatment in the United States, with $20.3 \%$ of individuals suffering from a disorder receiving treatment between 1990 and 1992 and 32.9\% receiving treatment between 2001 and 2003 (no difference was found in rates of prevalence between the two time points; Kessler et al., 2005). Even so, the majority of individuals with a diagnosable mental disorder are not receiving treatment. Ethnic disparities with respect to access to mental-health care among those in need are enormous. For example, African Americans are less likely to have access to services than are European Americans (12.5 vs. 25.4\%), and Hispanic Americans are less likely to have adequate care than are European Americans (10.7 versus 22.7\%; Wells, Klap, Koike, \& Sherbourne, 2001). These are illustrations from a much larger literature on disparities in mental health care delivery among individuals of minority groups (e.g., see www.mentalhealthcommission.gov/reports/Final Report/FullReport-04.htm). The lack of available services for most people and systematic disparities among those services have direct implications for models of treatment delivery. Interventions are needed that can reach many more people, but also with particular attention to select subpopulations.

\section{People Providing Services}

The problem of too few people receiving services and of groups being particularly deprived of such services might be conceived as a "person-power problem" in the mental health field. To oversimplify the argument, maybe more people are needed to provide the usual services. Three points convey why more providers alone may not be sufficient. First, the personpower problem stems in part from the geographical distribution of existing mental health professionals. The concentration of psychologists, psychiatrists, and clinical social workers in the United States is the greatest in highly populated, affluent urban areas and in cities with major universities (Health Resources and Services Administration, 2010; J.M. Richards \& Gottfredson, 1978). Thus, mental health professionals are not distributed advantageously to reach large swaths of people in need (e.g., rural areas, small towns).

Second, mental health professionals are unlikely to be able to reach the vast majority in need. In the United States, there are approximately 700,000 mental health professionals who provide services (Hoge et al., 2007). As we mentioned, estimates of prevalence indicate that approximately $25 \%$ of the population has at least one psychiatric disorder in a given year. With a U.S. population exceeding 300 million; $25 \%$ is approximately 75 million people. It is not necessary for these estimates to be precise to see that the number of individuals who can provide treatment - at least with the current dominant model of delivery - could not begin to reach the number of those in need. Doubling the work force might have little discernible impact given the number of individuals requiring services.

Third, it is not only the distribution and number but the profile of mental health professionals. Too few mental health professionals reflect the cultural and ethnic characteristics of those in need of care. For example, trends in ethnic minority representation in clinical psychology (e.g., doctoral enrollments and recipients and graduate department faculty) over two decades suggest that the proportions of ethnic minorities in psychology do not show comparable growth relative to that of the U.S. ethnic minority population growth (e.g., see Commission on Ethnic Minority Recruitment, Retention, and Training, 2008). Approximately $20 \%$ of doctoral degrees in psychology and current enrollees in psychology graduate training programs are of minority status, and approximately $6 \%$ of psychology faculty are of minority status (African American, Asian American, Hispanic American, Native American). The proportion of the U.S. population that comprises minorities, projected to be $50 \%$ by 2060 , is accelerating at a higher rate than trainees in their respective groups. Thus, the population of ethnic minorities in need of mental health services will increase at a greater rate than will the availability of ethnically matching professionals. Although treatments can be effective where there is not an ethnic match of therapist and client, in some cases that cultural component influences outcome (Griner \& Smith, 2006; Miranda et al., 2005). Even if an ethnic match is not needed for treatment to work, it can nevertheless present a barrier for the potential client who is considering treatment. Ethnicity and culture are not the only mismatch. Other groups based on geography (e.g., individuals living in rural areas) or select populations (e.g., the elderly) reflect a mismatch with a paucity of available resources and a plethora of need for mental health services (e.g., Hinrichsen, 2010; Institute of Medicine, 2008).

In light of the previous considerations, the inability to reach most people in need of services is not simply (or only) a personpower issue. Many of those in need of services cannot be reached for a variety of reasons (e.g., access, perceived and genuine barriers in obtaining treatment, insurance, rural areas), but one of them is our own view as mental health professionals 
regarding how treatment should be delivered. Invariably, more help is welcome. But, given the emphases of the current model of delivery, that alone is not likely to provide an increment of reduction on the burden of mental illness.

\section{Individual Psychotherapy in Relation to the Burden}

Many interventions will be needed to reduce the burden of mental illness and other facets of impairment. We begin with psychotherapy research for several reasons. ${ }^{2}$ First, there are many goals of psychotherapy, but salient among them is the treatment or amelioration of psychiatric disorders; social, emotional, cognitive, and behavioral problems; and stress (e.g., Dickerson \& Lehman, 2006; Mahrer, 1986; Weissman, Markowitz, \& Klerman, 2000). Thus, psychotherapy is a viable intervention for addressing significant mental health problems (e.g., anxiety, depression, bipolar disorder) in addition to other sources of impairment (e.g., stress). Second, psychotherapy research has progressed remarkably. As we mentioned, the emergence of EBTs is a particularly important advance. Generally, EBTs refer to those interventions that have carefully controlled research on their behalf. RCTs, careful delineation of the client sample, specification of treatment, and replication of the results by an independent investigator or team are among the commonly used criteria. Third, psychotherapy is a dominant model of delivering psychological services. By model of delivery, we refer to how the intervention is presented or provided. In this case, we refer to the dominance of individual, one-to-one therapy with a client (child or adult), family, or group (e.g., 8-10 clients). Psychotherapy plays a role in reducing the personal and social burden of mental illness. But the role it does play draws stark attention to what is missing if the burden of dysfunction is to be significantly reduced among those in need.

It is useful to highlight briefly the model of delivery of psychotherapy in historical context. That context conveys how the model has tacitly continued to dominate in therapy research and practice. Consider the enormous impact of psychoanalysis on the delivery of psychological treatment. For present purposes, three facets of psychoanalysis and its variations can be distinguished. First is the theory or substantive foci of problem development (e.g., related to psychosexual stages of development, superego, and variants) and of treatment (e.g., addressing transference). Second are the methodological features used in early work to support the key tenets. The anecdotal case study (e.g., Anna O., Dora, Little Hans) was relied on heavily. Third was the model of delivery of treatment, namely, one-to-one individual patient care, all flowing from a medical-patient care model. Psychoanalysis was not the first one-to-one psychosocial intervention (e.g., mesmerism, hypnotism), but it provided a prototype from which subsequent psychotherapies (and New Yorker cartoons of them) followed.

The contemporary history of psychotherapy research reflects the continual development and changes in substantive views that explain the onset of clinical disorders and the procedures required to effect therapeutic change. Many familiar examples within psychoanalysis and its variants (e.g., Jung, Kohut) and also from other views (e.g., self-theory of Rogers, reciprocal inhibition of Wolpe, cognitive therapy of Beck) and shifts in orientation (e.g., positive psychology of Seligman) illustrate the dynamic (not "psycho") nature of the field. Also, methodological advances have raised the bar for all treatment evaluation to include RCTs and increasingly more stringent requirements for how intervention studies must be conducted and reported (e.g., Consolidated Standards of Reporting Trials-CONSORT; Moher, Schulz, \& Altman, 2001). ${ }^{3}$

The third component, the model of one-to-one treatment, continues to dominate even as theories about the appropriate intervention focus (e.g., problem-solving strategies, mindfulness, and self-agency) have proliferated. The departures (e.g., treating couples, families) retain the focus on small individual units. The majority of EBTs retain this model of delivering treatment (Nathan \& Gorman, 2007; Weisz \& Kazdin, 2010). Also, the model dominates training in clinical psychology, social work, and psychiatry. In clinical psychology, for example, accreditation of graduate programs emphasizes clinical hours of treatment in individual therapy in graduate school followed by an internship experience. Treatment of groups (e.g., $8-10)$ and families are counted as well, but in relation to the focus of this article, delivering treatment by a mental health professional in person to small individual units are of the same ilk, namely, treatments with a very restricted reach.

Our comments are not a criticism of the model of individual therapy. One-to-one therapy will always have a place; individual crises and challenges in life are invariably at that level. Also, individual therapy contributes to the overall goal of reducing the burden of mental illness in at least three ways. First, it serves a small number of individuals with effective procedures, and that places it firmly in the portfolio of models of treatment delivery. Second, many of the scientific principles and processes (e.g., emotional memories, extinction, cognition) that serve as the underpinnings of individual therapy as well as the techniques themselves may inform other models of delivery. Third, some therapy techniques (as noted later) might be delivered in multiple ways, so the "same" treatment or very close approximations may vary in their accessibility and reach. Nevertheless, additional delivery models will be needed beyond the contributions of individual therapy.

\section{Developing a Portfolio of Models of Delivering Interventions}

Interventions that can reduce prevalence (cases with some dysfunction now) and incidence (new cases that emerge) are needed to reduce the burden of mental illness. Treatment and prevention work arm in arm. We emphasize treatment to convey key points and return to its integration with prevention later in this article.

Consider all individuals in need of psychological services (treatment) as occupying a pie chart. The goal in developing multiple models of delivery is to ensure that segments (slices) 
of the pie are covered (i.e., services encompass all or most in need - at least in principle). One might consider slices of the pie with the view that a given intervention or model of delivery may reach one slice, but that multiple models might be needed to cover more, most, or all of the slices. The pie notion is useful, but it does not convey the multidimensional needs of the population. There are many different reasons why people do not receive services, such as lack of access to facilities or practitioners, ethnic and cultural barriers, and many concrete obstacles (e.g., transportation, babysitting). No single model of delivery can be assured to circumvent all of the obstacles associated with a given subgroup or slice of the population pie.

Among the many characteristics that might delineate models of delivery, the ability to reach many individuals in need of services reflects the type of changes that are needed if treatment is to significantly reduce the burden of mental illness. We illustrate models of delivery of psychosocial interventions to convey some of the many options that might comprise the portfolio.

\section{Technologies}

The Internet. Unlike individual therapy, the use of various technologies to deliver treatment has the ability to reach a large proportion of the population in need of services. Among the technological options, there is a rapidly growing literature on the use of the Internet (e.g., Barak, Hen, Boniel-Nissim, \& Shapira, 2008; Dimeff, Paves, Skutch, \& Woodcock, 2011; Ybarra \& Eaton, 2005). The ability to reach a large segment of the population in need is nicely illustrated in an application to cigarette smoking, which is often a target of psychological interventions.

A series of Web-based intervention studies for smoking cessation conducted in English and Spanish have shown significant smoking termination rates through a standard smoking cessation guide and mood management course (Muñoz et al., 2006). An individualized, password-protected Web site provided access to the smoking cessation intervention to consenting eligible individuals and was used to obtain assessment data throughout the intervention. The intervention reached more than 4,000 smokers from 74 countries and was carefully evaluated (e.g., RCT, follow-up assessments). Studies of this program illustrate the potential for use of Web-based interventions and the ability to reach people in their homes. Once developed, such Internet-based administrations can be relatively inexpensive to implement and easy to maintain.

Telephone. As with the Internet, the telephone can be used to deliver mental health interventions for both individuals and groups (see Mohr, Vella, Hart, Heckman, \& Simon, 2008). Typically, "telemental psychotherapy," as this is sometimes called, involves administration of full sessions of therapy through scheduled weekly phone calls. This is much like the model of in-person psychotherapy, but because no face-to-face contact is necessary, it allows for remote administration of services. Interestingly, telephone-administered psychotherapies have lower rates of attrition than traditional individual psychotherapy (Mohr et al., 2008). Thus, phone-based treatment may not only broaden the population with access to therapeutic intervention, but also potentially increase the likelihood that clients will remain in treatment. Such a model has low cost and may even reach population segments to which Internet-delivered models may not have access.

One such illustration of the potential for telephone-based intervention is nicely demonstrated in "quitlines," initially developed to provide telephone counseling for smoking cessation through the U.S. National Cancer Institute. Tobacco users who call a quitline receive an empirically validated, standardized, and manualized intervention incorporating various services such as materials by mail, prerecorded messages, real-time phone counseling or a return phone call from a counselor, access to quitting medication, or some combination thereof (Lichtenstein, Zhu, \& Tedeschi, 2010). Quitlines have demonstrated tremendous reach, as they are currently offered in all 50 states and Washington, DC and have also been adopted and sponsored at the national level of various countries in Europe, Oceania, Asia, and South and North America. Although the content and structure vary across quitlines, the initial call typically lasts less than an hour, orients the client to the program, and establishes a quit date. Subsequent calls of 10 to $15 \mathrm{~min}$ are scheduled following the quit date over a period of 1 or more months with a frequency based on a relapse curve (Zhu \& Pierce, 1995). A staff with bachelor's- or master'slevel training typically delivers the counseling services, although computers drive much of the quitline counseling. An estimated $1 \%$ of smokers in the United States utilize quitline services each year (Cummins, Bailey, Campbell, Koon-Kirby, \& Zhu, 2007), and some states with increased marketing reach as many as $4 \%$ to $5 \%$ of their smoking populations in a single year (Swartz Woods \& Haskins, 2007). Quitlines have even demonstrated a special ability to reach underserved populations, as African American smokers are more likely than any other ethnic group to utilize these services and Asian immigrant smokers are as likely as European American smokers to utilize them (Maher et al., 2007; Zhu, Wong, Stevens, Nakashima, \& Gamst, 2010). This program highlights the advantages of models utilizing telephone-based intervention, namely, the potential to overcome various logistical barriers to treatment that exist for in-person individual psychotherapy. This and the brief, standardized, semianonymous nature of phone counseling administered by paraprofessionals greatly increases the accessibility and reach of the intervention.

Smartphones. Due to advances in technology, cellular phones are no longer simply mobile telephones for the sole purpose of making calls. Updates in these devices (e.g., GPS) provide new opportunities for methods of intervention and assessment (e.g., location of client engaged in homework assignments to overcome fear of open spaces; Boschen, 2009a, 2009b). The most commonly studied form of mobile phone intervention employs the use of short message service (SMS) or text messaging. One example of implementing an SMS-based intervention is nicely illustrated in an aftercare treatment of bulimia nervosa. Patients received weekly text messages for the 6 months following their release from inpatient psychotherapy. These messages provided 
feedback on their self-reported bulimic symptoms and consisted of both standardized messages and individualized feedback (Bauer, Percevic, Okon, Meermann, \& Kordy, 2003). Such an intervention has the advantages of low cost and effort, interactivity, individualization, anonymity, and widespread reach.

The increased use of multifunction smartphones provides additional prospects for methods of therapeutic intervention. These devices grant easy access to third-party applications that provide promising intervention opportunities. Although research exploring the use of such applications has only just begun, the implications for greater reach are remarkable. One example is the use of dialectical behavior therapy (DBT) for borderline personality (see Dimeff et al., 2011). A smartphone application called the DBT Field Coach provides a resource to clients, including specific skills and instructions, a $\log$ where individuals can track their use of skills throughout the day, access to uploaded supportive video and audio messages from their clinician and members of their support network, individualized motivational images when encouragement is needed, and a variety of means for enduring a crisis to prevent exacerbation of the situation, such as games and music to distract from intense emotional urges. Although this particular example of technology is used as an adjunct to the traditional individual therapy model, it nicely illustrates various resources that can be provided through mobile phone applications. Future research exploring the use of such devices may expand delivery to rely less or not at all on a professional therapist.

Another example that nicely illustrates the use of smartphone applications in therapy is an application known as Mobile Therapy (Morris et al., 2010). The application prompts users to report their mood levels throughout the day by indicating their mood on a touchscreen "mood map" and to report their levels on single-dimension mood scales for happiness, sadness, anxiety, and anger. The application subsequently provides mobile therapeutic exercises based in cognitive-behavioral techniques (such as breathing visualization, physical relaxation, and cognitive reappraisal exercises) as needed to cope with their stress and mood. A 1-month field study of the application demonstrated users' increased self-awareness and stress-coping abilities through the use of the therapeutic concepts.

These examples illustrate only a minute fraction of the opportunities that advances in networking and technology will provide for future therapeutic intervention. The constant updates and improvements in technology make it increasingly accessible to the public and provide new methods for collecting information and administering treatment. Most critical to the goal of reducing the burden of mental illness is the ability to reach those in need. Interventions that incorporate technology will reach far greater numbers of people than traditional psychotherapy and grant access to segments of the population that have been relatively inaccessible and neglected.

\section{Use of Special Settings}

Another model of delivering treatment takes advantage of special settings where those individuals in need are already present. An example of the potential to reach some such individuals is nicely illustrated in an intervention for cigarette smoking administered in a physician's office during a routine office visit. One intervention currently in use focuses on what physicians say to their patients during routine office visits. Physician visits are relatively brief (median $=12-15 \mathrm{~min}$ ) in the United States. During the visit, advice from the physician or nurse can have a small but reliable effect on smoking. The physician says something like the following to patients who are cigarette smokers: "I think it important for you to quit tobacco use now," or "As your clinician, I want you to know that quitting tobacco is the most important thing you can do to protect your health." The comments lead to approximately a $2.5 \%$ incremental increase in smoking abstinence rates in comparison with no intervention, as seen in meta-analyses of multiple RCTs (e.g., Rice \& Stead, 2008; Stead, Bergson, \& Lancaster, 2008). As a result of this effect, internal medicine practice guidelines now recommend that physicians provide specific advice to stop smoking. The example is instructive because it also conveys the importance of "weak" treatments. The intervention results in a small increase in the percentage of individuals who became abstinent. Small effects on a large scale (affecting many people) provide an important complement to other models of delivery.

This example might raise concerns that many cigarette smokers may not attend routine physical exams or may not admit to smoking and therefore not receive the message. Yet the task is not to have one intervention reach everyone. We do not possess the psychological equivalent of fluoridation that can be poured into a stream from which mental health flows. Rather, we only wish to illustrate interventions that have the ability to reach individuals who might not seek intervention or not have readily available access to care. Numerous other special settings may be identified and used for the goal of reducing the burden (i.e., schools, community centers, welfare offices, family settings, to name a few).

\section{Opportunities for Nonprofessionals}

The focus on everyday settings underscores opportunities for nontraditional providers to administer interventions that can improve mental health. This is not an effort to substitute high-school students, fellow parents, or work colleagues for professional therapists. Rather, in developing a portfolio of interventions, there are multiple opportunities to intervene both for prevention and treatment and these can reach many people in need. For example, in one intervention aimed at reducing rates of sexually transmitted diseases (STDs) among African American adult males, a lay health adviser administered a single-session sex-education program that reduced rates of unprotected sex and number of sexual partners and increased condom use. This intervention resulted in reduced rates of STDs in participants (Crosby, DiClemente, Charnigo, Snow, $\&$ Troutman, 2009). This program was brief, reached a portion of the population with traditionally little access to therapeutic intervention, and was administered in a clinic-based setting. 
Parents have played a role in treatment administration to other parents and families as well as their own children (Hoagwood et al., 2010). For example, a parent-based intervention focused on reducing binge drinking in first-year college students (Turrisi, Jaccard, Taki, Dunnam, \& Grimes, 2001). Parents were given a 35-page intervention handbook to read, which contained all of the necessary instructions for implementing the communication-based intervention with their precollege adolescents. Unlike the traditional psychotherapy model, this example highlights relatively low cost and effort and ease of administration within a home setting without the direct involvement of professionals and with very little training.

\section{Self-Help}

Self-help is a set intervention in which the individual takes control and implements an intervention on his or her own. There are variations that reflect a continuum of external support, including complete independence; group support; and minimal to full-time aid from volunteer, semiprofessional or professional help (T.M. Harwood \& L'Abate, 2010). Self-help interventions use various media (i.e., audio recordings, books, video, the Internet) to address numerous mental health concerns. For example, an Internet-based self-help treatment for panic disorder, consisting of five modules over 5 to 8 weeks, was associated with reductions in panic frequency and distress during panic attacks (J.C. Richards \& Alvarenga, 2002). This particular self-help intervention was solely self-directed and included psychoeducation regarding anxiety disorder and coping skills. Once established, an intervention like this can reach many individuals and at a low cost.

In general, self-help interventions comprise an immense literature encompassing numerous approaches at various degrees of involvement by professionals. For example, an entire selfhelp treatment genre is based on writing. Distance writing, in the form of open-ended journal entries; autobiography; and expressive, programmed, or dictionary-assisted writing, allows for the exchange of information between professionals and participants so that help can be given without seeing one another face-toface. A second example of the diverse approaches to self-help is that of group self-help. There are many options for group self-help interventions, as evident in support groups consisting of people facing a similar challenge and attempting to overcome their shared adversities (Davison, Pennebaker, \& Dickerson, 2000). Group self-help interventions also can vary in degree of involvement of trained professionals, and some are even led by a fellow group member.

Countless self-help books, known as bibliotherapy, have been written for a wide range of mental health problems. Also, there are various Web-based self-help and informational resources for diverse problems, some of which may even incorporate online support groups. ${ }^{4}$ Many self-help treatments are now evidence-based interventions with comparable effects (effect sizes) to those obtained with individual therapy (T.M. Harwood \& L'Abate, 2010). With so many self-help resources available for a wide variety of conditions, including anxiety and mood difficulties, eating disorders, addictive behaviors, personality disorders, and severe psychopathologies, the model of self-help is an excellent addition to the portfolio of models of treatment delivery. Self-help has the potential to reach individuals in need and reduce many barriers (e.g., geographic and financial barriers) associated with more traditional therapistdelivered interventions.

\section{Media}

The media (i.e., radio, television) can be an effective way to implement widespread intervention with great capability to reach large segments of the population. Entertainment education is a prominent example of how to exert social change on critical issues including family planning, adult literacy, HIV/ AIDS prevention, sexual abstinence for adolescents, parenting, and promoting a sustainable environment and mitigating climate change (Charles, 2009; Singhal, Cody, Rogers, \& Sabido, 2003; Singhal \& Rogers, 1999). The process begins by studying individuals within a given culture (e.g., surveys, focus groups) and developing characters for an extended radio or television drama series (depending on the medium available to the community) that reflect local culture and people in their daily lives. The characters take on different roles, deal with daily challenges of life within the culture, and model social change on the critical issues.

The goal is to achieve concrete change within individuals, communities, and societies. For example, one of the early applications in Mexico focused on family planning and efforts to reduce fertility rates (Singhal \& Rogers, 1999). Family life, marital relations, and the daily drama and stressors were conveyed in detail as the televised series unfolded. The fictional family gained control over their lives and benefitted from family planning - all in realistic episodes. In terms of impact, sales of contraceptives in the community rose dramatically, and there was a $34 \%$ drop in birthrates over a 5 -year period. Similar results were obtained in Kenya. More generally, the model has been used throughout the world on other social issues and has produced widely engaging shows. Ratings of shows are high, viewers or listeners become involved with the characters, and there is genuine change on the targeted social behavior, rather than just increased awareness. An effort with a focus on largescale mental health problems, handling stress, substance use, or depression could be targeted to various groups and geographical regions and could have widespread reach, especially as this strategy has been successful on TV as well as radio.

\section{General Comments}

Our illustrations are not intended to be comprehensive. We have taken off-the-shelf, currently available interventions - not all of which have strong evidence on their behalf. Most of these are not routinely included in reviews or evaluations of EBTs. Also, the models of delivery usually are omitted from graduate training in psychology, psychiatry, or social work and are not easily available to individuals in need of services. Our goal in mentioning 
diverse models of delivery is to prompt a slightly different way of thinking about psychosocial interventions. The focus is not on one ideal model of delivery, but on dovetailing multiple models of delivery, each incorporating various characteristics that will allow them to reach many individuals in many different ways and ultimately to reduce the burden of mental illness.

We have noted that most EBTs are based on the delivery of individual therapy. This model of delivery is not very helpful in reaching the many individuals in need. However, developing EBTs may potentially contribute to the goal of reducing the burden of mental illness. The scientific principles underlying various individual psychotherapies as well as the techniques that derive from them can then be extended in a programmatic way to move from intensive, costly, and individual case application to versions that are more population based.

An excellent example is the Positive Parenting Program (Triple-P), a parenting program developed in Australia to treat disruptive behavior in preschool children (Sanders, 2008; Sanders \& Murphy-Brennan, 2010). Early studies demonstrated efficacy in applications with individual families. Over a period spanning 25 years, efforts were made to develop brief and cost-effective versions of the program, ways of delivering treatment through groups, and flexible delivery through telephone consultation and the media. The range of interventions available from this one "treatment" encompasses versions of the program that can be intensively provided to individual families or provided as preventive interventions via media widely available (e.g., DVD, online). The transition involves more than just varying how treatment is presented because new tasks and challenges emerge as treatment moves to the community from the treatment setting.

We raise this example as a successful case of starting with an individual therapy model and using the treatment to develop multiple models of delivery. The challenges of moving a given treatment from an individualized version to something available to more people on a large scale will vary as a function of the clinical problem, treatment, and age of the clients. Even so, there may be some standard challenges or an approximate template as a guide to help move treatment through different models that vary in their reach.

Among the major challenges is identifying how an intervention achieves change. Although there are many EBTs available, there is little understanding of the mechanisms of change (i.e., precisely how they work; Kazdin, 2007). Understanding mechanisms of action may be extremely important when trying to develop different models of delivery of a given treatment that vary in intensity, mode, and agent of delivery. Knowing what the essential ingredients are as well as how they work (mechanisms) will ensure that these critical facets are not unwittingly sacrificed as the treatment is scaled up, monitored less closely, and abbreviated.

\section{Critical Issues Central to the Portfolio}

If a central goal of psychological interventions is to reduce the burden of mental illness, we question whether current advances in treatment will meet that goal and consider what more might be needed to have an impact. A portfolio of intervention models is emphasized for sensitivity to the diverse individuals in need and the contexts, settings, and circumstances required to reach them. Even so, it would be naive to imply that the field merely needs to get a bigger bag of intervention delivery tricks to solve the national (and international) burden of mental illness. There are additional and complementary considerations we highlight as well.

\section{Prevention}

We have focused on treatment and specifically psychotherapy because of the enormous attention these receive in research, practice, and clinical training. Prevention is pivotal, and here too the same points can be made - namely, that a portfolio of preventive interventions with various models of delivery is needed. There are some ways in which prevention is further along conceptually, insofar as the portfolio of interventions (e.g., targeted, universal), the timing of interventions (e.g., prenatal, preschool), intervention settings (e.g., home, schools), and selection of samples (e.g., risk factors) recognize the need for multiple interventions delivered in multiple venues and contexts. What we add to this is the integration with treatment and the portfolio concept of identifying preventive interventions that vary in their reach and other characteristics (e.g., cost, effort). Reducing the burden of mental illness will depend on avoiding onset or limiting severity of onset and, by doing so, reducing incidence and the need for treatment. The portfolio idea would be beneficial for conceptualizing the task of prevention because it begins with who ought to be reached in the population, what interventions are likely to accomplish that for various groups, and what the effects are.

The treatment and prevention agenda are shared, complementary, and essential to integrate. The treatment question underlying this article is "What models of intervention delivery will help reach the population of individuals in need?" The prevention question is "What interventions or experiences can be provided for persons who are or might be at risk that can avert the onset or severity of some condition?" Decreasing prevalence and incidence are important for more than just the goal of reducing the burden of mental illness. Theory and principles that underlie current interventions as well as the techniques that derive from them might have variations applicable to both prevention and treatment. Many of the delivery methods (e.g., use of the Internet, parent-to-parent delivery) may be shared as well.

In short, we omitted prevention to focus our argument on the task of developing a portfolio of treatment delivery models. To reduce the burden of mental illness, we must expand our conceptualization of interventions and models of delivery. However, that thesis is equally applicable to prevention and treatment. We focused on treatment because of the advances in evidence-based psychotherapies and their still restrictive model of delivery. 


\section{Assessment}

The goal of reducing the burden of mental illness begins with better assessment to monitor mental illness and impairment nationally (i.e., some measure of the mental health of the nation). This would provide ongoing information for tracking changes in mental illness and its burden over time across cohorts and across possible social influences that might affect that baseline. There is a need for a national database on mental illness that allows comparisons over time - a point others have well articulated (e.g., Bickman, 2008; Chorpita, Bernstein, Daleiden, \& The Research Network on Youth Mental Health, 2008). There are many models that are in place to monitor mental health changes over time. As a prominent case in point, the National Comorbidity Study, which samples the mental health status of thousands of adults and youths, provides data on incidence and prevalence and encompasses several countries in conjunction with the World Health Organization (www.hcp. med.harvard.edu/ncs/). Another example is the Monitoring the Future Study, which assesses behaviors, attitudes, and values of school students (http://monitoringthefuture.org/). The project began in 1975 and has provided data on drug, alcohol, and cigarette use nationwide and involves approximately 50,000 students (8th, 10th, and 12th grade) annually. Finally, the Institute for Health Metrics and Evaluation (University of Washington) focuses specifically on evaluating data on health indicators including prevalence of major diseases and effectiveness of health programs (Murray \& Frenk, 2008). Mental health and major psychological sources of impairment would be a natural extension of this latter effort, given that mechanisms for rigorous evaluation are already in place. In short, there are precedents and methodologies for obtaining the requisite data.

A national database is a fundamental step for decreasing the burden of mental illness because it provides a baseline to better establish the extent of the burden and whether there are changes over time. We have emphasized psychosocial interventions to reduce the burden of mental illness. However, there is no single modality of intervention or discipline that has claim to the range of factors that might have such impact. Interventions, natural and human made, from climate, pollution, and natural disasters are known to have deleterious impact on physical and mental health (e.g., Berglund, Lindvall, \& Schwela, 1999; Bouchard et al., 2009; Reacher et al., 2004; Satcher, Friel, \& Bell, 2007). With a goal of reducing the burden of mental illness at a national level, understanding factors that might contribute could also mobilize multiple disciplines (e.g., education, sociology, social policy). A national database would provide opportunities to generate and test hypotheses about influences (e.g., economic, social) that might be understood and possibly harnessed to improve mental health.

Assessment issues emerge at a more concrete level in developing a portfolio of interventions. Outcome assessments in psychosocial intervention focus on the domain of interest. Treatments for depression, for example, include multiple measures of depression and perhaps related domains as well (e.g., stress, physical symptoms). In developing a portfolio of interventions, outcome measures remain important. However, measures might also focus on characteristics of the treatment delivery model. Characteristics of treatment-such as what groups in need can be reached and when (developmentally) and where (setting) the intervention can be delivered-become important. The arbiter of the value of a treatment is not necessarily in its effect size on outcome measures but where that intervention fits in a broader portfolio to help reduce the burden of mental illness.

Measuring characteristics of treatment that relate to delivery is not at all new. Cost of interventions and cost-benefit analyses are examples of measures that reflect on the utility of interventions (e.g., M.G. Newman, 2000; Yates, 1995). Although these measures are not routinely used in treatment studies, they have been used on multiple occasions (e.g., Gabbard, Lazar, Hornberger, \& Spiegel, 1997; Krupnick \& Pincus, 1992). Cost is one important measure for building a portfolio of interventions. Other dimensions also relate to the reach of interventions, such as "therapeutic effort" (dose, degree of restrictions placed on the client, and cost of repeated episodes of the disorder; F.L. Newman \& Howard, 1986). The categories or dimensions need to be devised along with operational definitions that permit some classification or characterization. The goal is to develop interventions that overlap along some dimensions but purposely do not overlap in other ways. Consequently, characteristics of interventions (e.g., how they can be used, their scale of application, when during development they might be applied) are no less important than the usual outcome measures.

\section{Contextual Influences on Reducing the Burden of Mental IIIness}

There are many influences that contribute to, determine the effects of, and limit the delivery of mental health services. There are enormous barriers for persons in need that interfere with receiving services. Some of these are perceived; others are real. Their net effects are similar. Many of the barriers involve health care policy, law, legislation, limits of insurance and third-party payment, competing interests of different stakeholders in health care, and politics. These are important to acknowledge for at least two reasons. First, collaborations will be needed with many organizations and interest groups to effect changes in policy and legislation that can influence accessibility to mental health services. As an obvious and historically important case in point, recent health-care legislation in the United States extends health coverage to tens of millions of uninsured persons, reduces health care costs for many with and without insurance, and ends insurance practices considered by many to be discriminatory. ${ }^{5}$ Such legislation can alter the reach and availability of services, as well as the nature of these services (e.g., psychological services in the context of community-based health-care teams). Thus, we recognize the healthcare delivery system is dynamic and an important influence in conceptualizing what services will be provided to whom. 
Second, the contextual influences that drive mental health services are not necessarily immutable or givens, but they might well be influenced by developing novel models of service delivery. The development of multiple models of delivery that vary in cost, disseminability, and ability to be delivered for large-scale application might well influence contextual factors (e.g., policy, law, reimbursement) that seem outside of the control of any one discipline. For example, early intervention delivered by lay persons or via large-scale Internet applications might change the nature of insurance coverage, reimbursement, and the costs of delivering more intensive services. Insurance companies might well be willing to cover more intensive psychological intervention services for those individuals who have not responded to more readily available and less costly evidence-based interventions.

The possibility that better, different, and more diverse intervention models might reduce barriers to treatment is speculative. It is better to acknowledge that psychologists and other mental health professionals do not control or are not likely to have great impact on key policy influences related to providing services. That does not gainsay the areas over which mental health professionals do have control. The intervention research agenda could be modified to focus more on a portfolio of interventions that could reach more people and seek to reduce mental illness and its burden. Mental health professionals could readily influence implementation of preventive and treatment services if there were more to implement and more ways to deliver those services.

\section{Potential Tensions Within Mental Health Professional Training}

Each of the mental health professions has a model of clinical training that combines academic and practical experiences. Training and requirements for clinical work may actually interfere with developing and implementing a portfolio of models of delivering treatment. This can be illustrated by commenting on three issues within clinical psychology: accreditation, determining who is allowed to deliver services, and reimbursement and jobs. First, within clinical psychology, accreditation of graduate training programs influences greatly the model of treatment delivery. Individual therapy and its closely related variations (family, group, couples therapy) are required. This model of delivery reflects what "counts" as legitimate training and permits one to acquire a license to practice in states and provinces within the United States and Canada. Thus, currently within the profession of psychology, a portfolio of models of delivery is not quite in keeping with requirements of training by tradition and by required courses, although there are no prohibitions against novel treatments.

We mention clinical training merely to acknowledge a potential barrier in expanding models of treatment delivery. Any broad intervention portfolio taken up by researchers may need to be reflected in clinical training. Otherwise, interventions would be developed that ultimately would not be used in practice. Accreditation requirements are very slow to change, and merely developing new models of delivery alone will not be sufficient to ensure they will be taught in training programs, allowed by licensing boards to count as relevant experience, and then delivered in practice.

A second potential source of tension in advocating a portfolio of models is the question of who would be involved in administrating interventions. Some of the interventions would focus on preventing onset of dysfunction among at-risk groups. For example, an intervention might be conducted at home or at school long before any treatment was needed. But consider interventions where treatment is needed, because this better conveys the professional issues. It is heresy within psychology to mention that one does not need to have a $\mathrm{PhD}$ to deliver effective or evidence-based individually tailored treatments. Indeed, it would be difficult to support empirically that $\mathrm{PhD}$ trained individuals are more effective than those with less training. At best, experience and possessing a doctoral degree would be moderators of treatment - that is, they might influence outcomes in some situations. Even if there were strong evidence that a $\mathrm{PhD}$ improved outcomes, it would still mean there probably is no difference as a function of academic degree in many other circumstances. With a broad portfolio of interventions, there are opportunities for peers, lay people (parents), the media, teachers, bachelor's- and master's-degree-level individuals, and self-help to play a role in reducing the burden of mental illness. Actually, these are not "opportunities"- a large set of them is likely to be essential to reduce the burden of mental illness. Thus, an impediment to reducing the burden of mental illness might be assumptions and restrictions on the range of individuals who are allowed to provide services in one form or another.

A third potential source of tension stemming from the advocacy of a portfolio of models regards jobs and reimbursement for clinical services. Currently, strong advocacy and lobbying efforts focus heavily on obtaining reimbursement for $\mathrm{PhD}$-level practitioners engaged in the traditional model of providing services (www.apapracticecentral.org/advocacy/index.aspx). It is natural for a profession to set standards for training (e.g., accreditation, core curriculum), protect its name (e.g., use of "psychologist"), and worry about restricted and unrestricted trade practices (e.g., who is allowed to provide, charge, and be reimbursed for mental health services). Our article does not lobby for eliminating any particular practice; however, it does draw attention to the need for more options for service delivery, given the large majority of unserved individuals in need. We begin with the goal of reducing the burden of mental illness based on psychosocial interventions (i.e., those interventions to which research is or might be devoted). This point of departure opens many options, but it also sacrifices protective practices that focus on the question of who gets to deliver treatment. Rather, the goal begins with how delivery of services can be optimized to reduce the burden and what innovations in services would be needed. One would hope that there is no clash between reducing the burden of mental illness and retaining individual therapy as a model of treatment delivery as delivered by doctoral-level individuals who have accredited clinical 
training. Perhaps there are new roles and opportunities for doctoral-level trained clinicians (e.g., supervising and monitoring a network of others who vary in training) that would still give the highest priority to reducing the burden of mental illness. Psychology can play a major role in providing a portfolio of interventions given the theory and research about human behavior from which to draw, even if the actual delivery of the full range of interventions has many agents of administration.

\section{Collaborating With Other Disciplines: Brief Illustrations}

Reducing the burden of mental illness involves challenges well beyond developing a broad portfolio of treatment delivery models. The goal will require collaborating with other disciplines, in part because of the complexity of the influences to be considered in providing services under many different conditions (e.g., economic) and contexts (e.g., cultural). Collaboration in the sciences has increased (Cacioppo, 2007; Kliegl, 2008) and now collaborative work or team science exerts greater impact than work of individual investigators (Wuchty, Jones, \& Uzzi, 2007). Similarly, collaborations to reduce the burden of mental illness are likely to increase the impact of any single profession. Of course mental health professionals (e.g., in psychology, psychiatry, social work) already collaborate with each other to provide treatment services. Yet the collaborations we are referring to encompass other disciplines and strategies that are beyond standard practice. Consider briefly a few disciplines and approaches and how they might contribute.

\section{Mathematical Modeling}

With current treatments and an expanded portfolio of delivery models, one might ask, "What is the best way to allocate resources to reach those individuals and groups in greatest need or to have the greatest reduction in the burden of mental illness?" Math can be quite helpful in modeling the challenge and potential solutions. The point can be illustrated in the context of controlling epidemics, responding to a bioterrorist attack (e.g., smallpox), and deploying vaccines to keep illness to a minimum (e.g., Hughes, Garnett, \& Koutsky, 2002; E.H. Kaplan, Craft, \& Wein, 2002; Magal \& Ruan, 2008). Critical variables such as how contagious the disease is and for what duration, how many contacts individuals have, what groups are at greatest risk for contracting or spreading the disease, how long it takes for the vaccine to take effect, and other such variables can be modeled in relation to how to minimize death and to eradicate the disease.

In relation to psychological treatment, there are three major intervention challenges to consider: preventing onset of psychiatric disorder, treating acute disorder (e.g., trauma from a catastrophic experience), and treating chronic and episodic disorders (Ulmer, Bruno, \& Burke, 2010). The methods for intervening with better mental health services will vary as a function of these challenges and more specifically the types of clinical problems, different onsets and durations, courses, and amenability to a given type of intervention that varies in cost, ease of administration, ease of delivery on the scale needed, resources to provide these interventions, and likely effectiveness, to mention some of the factors. These factors could be modeled to help guide the field in where to place the emphasis not only in delivering but also in developing interventions.

There are several areas in which math modeling plays a critical role in solving complex problems. For example, in operations research, math and statistics are used to achieve goals while maximizing the desired outcome and minimizing loss or risk. Probability theory, game theory, graph theory, decision analysis, queueing theory, and more are brought to bear to achieve some goal (e.g., Hiller \& Leiberman, 2005). Early applications solved problems of deploying weapons in war and focused on decision making for complex but very practical problems. Operations research extends the point here about drawing on math but also statistics and many related modeling tools used in other disciplines to solve policy and complex application problems. Providing better mental health services is not only or even primarily a math or operations research problem, but there could be great benefit in drawing on these and related areas in deciding priorities for developing and deploying interventions.

\section{Technology}

We highlighted the use of technology in the development of a portfolio of treatment delivery models. Arguably technology could have the greatest impact on psychological interventions in the coming years. Psychotherapy research already draws on technology, especially the delivery of direct services over the Internet for many clinical problems (e.g., anxiety, stress, pain, phobias) for the treatment of adults (e.g., Barak et al., 2008; Ritterband et al., 2003; Rochlin, Zack, \& Speyer, 2004). However advanced many of these seem, this area is probably at a very early stage because of the development of the technology itself.

Three critical uses of technology are important to mention in relation to improving clinical services and their reach. First, technology can deliver interventions and reach places beyond the reach of brick and mortar services. Even though some of these treatments are still intensive (e.g., requiring a trained therapist), not all of them are. Second, and especially relevant to the portfolio, technology might well permit treatment with less, little, or no therapist contact. Clients can access materials that can promote therapeutic change with little or no therapist assistance. Finally, technology will permit refined assessment and feedback both to patients and clinical services. Electronic devices to record functioning in everyday life are already in use in such areas as the study of sleep, mood, social interaction, and speech (e.g., Hasler, Mehl, Bootzin, \& Vazire, 2008; Pentland, 2008). Better assessment can greatly enhance interventions in targeting both when and to whom an intervention is provided.

Technology can be exploited much further, and collaboration with leaders in technology applications could benefit the 
development of a portfolio of models of delivery. The creativity of video games may increasingly be applied to treatment or preventive regimens and be made readily available. Similarly, a library of virtual evidence-based interventions for psychological conditions is hardly a conceptual or technological leap. The initial reaction is that technology will never substitute for a "real" person. An aficionado of robotics might argue that, but the better point is that technology is not competing with a real person. It is directed toward the goal of reducing the burden of mental illness, and in this regard it can make a contribution to a portfolio of delivery models that is without peer.

Technology for intervention delivery and assessment will advance and will contribute enormously to mental and physical health. Smartphones are currently being used in everyday life, but as technology moves to increasingly brilliant phones, assessment and intervention possibilities increase. As for assessment, subjective experience and biological indices of psychological states (e.g., via breath, blood flow, electrophysiology, smells) could be fed back to some clinic but also could be fed back to the device with the client and activate some intervention. Perhaps a virtual teddy bear to hug in times of crises would reduce impairment ever so slightly. "Ever so slightly" can make a difference in determining whether an individual goes off the deep end or wades in the shallow water until a crisis passes.

\section{Diet and Nutrition}

The credibility of the role of diet in the etiology and treatment of psychological and psychiatric dysfunction has suffered from faddish diets, quick cures for desperate parents and clients, and, at best, checkered evidence. It is still the case that diet "cures" are readily available on the Web (e.g., for attention-deficit/ hyperactivity disorder, autism, and dyslexia) despite the absence of evidence and multiple empirical diet challenges that show little or no impact. With that background, one must tread carefully. Yet diet and nutrition continue to involve increasingly sophisticated lines of empirical research (e.g., alternative medicine, cellular microbiology of nutrition). Also, mechanisms of action of critical influences (e.g., conversion of diet to minerals to neurotransmitters; cell trafficking and transport) and the ability to assess these mechanisms in a more finegrained fashion have changed the nature of research. It is very plausible that diet, nutrition, vitamins, and minerals affect critical psychological processes and can be harnessed to influence mental health and illness (see B.J. Kaplan, Crawford, Field, \& Simpson, 2007).

From the standpoint of this article, the ability of diet to reach a large segment of people would make this an excellent addition to a portfolio of models of delivery. Of course, one would want strong evidence that nutrition and mental health were connected in relation to risk factors, etiology, and treatment. There are many intriguing leads already. For example, pesticides in one's diet (e.g., especially on fresh and frozen fruit) have been implicated in the onset of attention-deficit/hyperactivity disorder (Bouchard, Bellinger, Wright, \& Weisskopf, 2010). As another example, several years ago, a review of fatty acid supplements for psychotic disorders suggested that the research was promising (Joy, Mumby-Croft, \& Joy, 2003). More recently, a randomized and placebo controlled trial suggests therapeutic effects of fatty acid supplements on psychoses (Amminger et al., 2010).

The roles of diet and nutrition in mental health remain to be developed. Any dietary component that could be harnessed and shown to prevent, treat, or attenuate acute or chronic psychiatric symptoms would be a valuable addition to the portfolio of treatments. We are not suggesting that diet is the answer or that the new New Yorker therapy cartoon should be a drive-through fast-food place to treat serious psychopathology. We are suggesting that if the focus is on reducing the burden of mental illness and associated conditions, there are several partners in this enterprise. Nutrition might well be one. The arbiter is evidence and allegiance to the goal rather than allegiance to a profession or a restricted model of treatment delivery.

\section{Epidemiology and Public Health}

These two linked disciplines are obvious partners because they focus on the distribution of dysfunction (e.g., disease), the factors involved in risk and prevention, and population-based interventions. Reducing the burden of illness and disease is central to the goals, and drawing on that orientation will be pivotal to work in the mental health professions. As an example of a public-health approach, the Office of Disease Prevention and Health Promotion has delineated a national goal: Healthy People 2020 (www.healthypeople.gov/). This initiative sets national objectives for promoting health and preventing disease. Every 10 years, the initiative draws on what has been learned from research regarding health and uses that as a basis for setting priorities. The public sector and various stakeholders are involved to craft the policy and to promote health. Major agencies also illustrate the public-health approach. Prominent among these is the Centers for Disease Control, which takes a population approach to prevent illness and to improve the quality of life (www.cdc.gov/). Among the key features of epidemiology and public health and the specific examples mentioned briefly here are interest in evaluating health and disease, the factors that predict onset and can be used to identify groups at risk, the development and testing of intervention strategies, and encouragement to move to policy or widespread adoption where possible. They also recognize the disparities in health-care delivery and those who are not served.

A public-health approach is central to a portfolio of interventions. The field needs population-based interventions that can reduce the burden of mental illness through prevention as well as treatment. However, population-based interventions alone will not be sufficient; many individuals in need will be missed, many who do receive the intervention may not respond, and many who respond may not respond well enough. Also, public-health approaches often consist of providing information to the public. Psychological science can add to this approach by drawing on theory and research (e.g., message 
framing, social norming, focusing illusion) that might be applied to optimize changes in attitudes, behaviors, decision making, and subjective experience.

A public-health perspective sensitizes the field to an approach to interventions that warrants more influence on what is done in psychology. At the extreme, a very costly intervention could be identified (e.g., individual, weekly, in-person psychotherapy) that can only be applied on a small scale to a very select few of the many in need. In a portfolio of intervention models, there is a need and place for that intense focus. However, the mental health professions must also identify interventions that are effective and could be provided on a greater scale to larger groups (e.g., communities, large patient groups). There are examples in which public-health (population) perspectives are being encouraged to address family interventions for parenting and domestic violence (e.g., Sanders, 2008; Sher \& Halford, 2008). Advances in technology, mentioned earlier, also provide a window to increasingly larger extensions of psychological interventions to the public at large. Collaborations with public health at the outset of intervention development could enhance development of the portfolio of interventions.

\section{General Comments}

We have highlighted a few of the many disciplines and areas with which we might collaborate in the effort to reduce the burden of mental illness. There are many other disciplines (e.g., economics, business) and topics (e.g., exercise, meditation) that could have been included. Our illustrations are to advocate for partnerships rather than to limit who those partners might be.

Reducing the burden of mental illness can profit from many basic and applied areas of psychological research. Intervention research is the work most immediately directed to the goal, yet intervention research alone will not accomplish the goal of reducing the burden of mental illness. For example, producing "new and improved" EBTs may do little if still administered as individual therapy in ways that systematically exclude most of the population in need and especially those most in need. Partnering with other areas can help shape the agenda and provide theory, research, and methodological tools that can guide development of models and how they can be deployed to reach those in need.

\section{Conclusions}

This article began with the view that psychosocial interventions directed toward mental illness and health should primarily focus on the reduction of mental illness and the impairment associated with social, cognitive, emotional, and behavioral functioning. The prevalence of dysfunction is relatively high, and most people who might benefit from services for their dysfunction do not receive care. Additional resources in terms of person power might help. However, the dominant model of treatment delivery in clinical practice focuses on in-person treatment provided to individuals or relatively small units (groups, family, and couples). The model constrains the ability to reach individuals in need, even if the number of mental health professionals doubles. Developing EBTs and placing these in the hands of practitioners remain laudable accomplishments and goals, respectively. Although we as researchers and clinicians ought to perfect our individual treatments, understand how they work, and disseminate them, much more attention is needed to those models of delivery that can reach the majority of people in need; that will require a portfolio of models of delivery.

The goal of developing a portfolio of models of delivery expands on the traditional and current research agenda. Much of research in the context of therapy has compared treatment against treatment as usual or has compared different treatments to see which one is better or best. Indeed, the federal Comparative Effectiveness Research agenda highlights and underscores this as a current priority (www.hhs.gov/recovery/programs/cer/ index.html). When two (or more) interventions have identical or nearly identical goals and are very similar in their characteristics (e.g., to whom they can be applied, for a given cost, on a given scale), invariably there is the question of which one is better. If they are equally effective, there is the question of whether one has some other advantage (e.g., fewer side effects).

The research agenda implied by the development of a portfolio of delivery models shifts the current focus a bit. Evidence that an intervention is having an effect is still needed. However, interventions that vary widely in their reach, focus, costs, effects, and other dimensions are crucial. Separate components of a portfolio that will not be directly comparable are needed. Each is useful in relation to the overall goal (reducing incidence or prevalence), but they will focus on different windows of opportunity to effect change and will be targeted to different people, in different settings, and so on. Direct head-to-head comparisons will not be as relevant if there are different interim goals needed to achieve the longer term goal of reducing the burden of mental illness.

The evaluation of some intervention research may change slightly. For example, EBTs often seek large mean effect sizes (ES; e.g., $d>.80$ a la Cohen, 1988). With a broader portfolio of models of delivery, strong ESs are not always the first consideration. An intervention with a larger ES is not invariably better than one with a smaller one. An intervention with a weak but reliable effect that can reach large numbers with little cost would be worth having and could only be bumped out of place (think of the Olympic sport of curling) by another intervention with a greater ES that addressed the same population, cost, and so on. Also, it is quite possible, even in this context, that both treatments are kept as viable options because they reach a slightly different group among those in need.

In addition to the research agenda, conceptual work is needed to address the broad focus on reducing the burden of mental illness. The task is to consider how interventions might relate to each other, how they might be sequenced in a step-like fashion, to whom they are applied, and when. For example, reducing the burden of depression requires consideration of different ages of onset, different types of depression, and different 
durations of depression. It would be helpful to develop broader concepts for where to begin here. If the goal were to reduce the burden of unipolar depression, one consideration would be to determine the windows of opportunity that might be used across the developmental spectrum for preventing onset and reducing impairment of those who are currently depressed. Formal consideration of the battleground and where first, best efforts are likely to have impact would be an important step. By formal consideration, we refer to drawing on specific models (e.g., math modeling, operations research, strategic planning) that help guide the agenda.

Mental health professionals might continue to refine individually delivered therapies, perhaps especially if they generate techniques or principles that can be applied in different ways. Yet it is necessary to make progress in new ways with a focus on the goal of decreasing mental health problems. Mental illness is an enormous burden. Anyone with a family member who suffers from mental illness knows all too poignantly the personal costs, the suffering and pathos of those who experience the dysfunction, and the toll of the necessary care for a loved one directly suffering. From a societal perspective, public and private agencies at every level know the burden of economic costs. Most people with mental illness are not being served. The challenge is determining what is being done in applied intervention work to reduce mental illness and extend care to those in need. Current models of delivery need to be expanded to reduce the burden. Continued proliferation of treatments delivered in a way that cannot reach most people in need ought to reconsidered. The goal of this article was to draw attention to a neglected, albeit central goal of psychosocial intervention research - namely, the goal of reducing the burden of mental illness. The research, practice, and training agenda ought to integrate this goal of intervention work with the needs of the national and international community.

\section{Editor's Note}

If you are interested in commenting on Kazdin and Blase, you can submit a 200-word abstract to the Perspectives portal at http:// mc.manuscriptcentral.com/pps by February 14.

Please submit the "type" as "commentary." You will have to submit the abstract both as "abstract" in Step 1 and "main document" in Step 6. Editors will read the abstracts and invite a few authors to write full comments. These will be published together in a subsequent issue along with a reply by the authors. Criteria for selection include likely influence of the comment/critique, interest to a broad readership, and importance of the issues raised. Also, the Editors will select a set of proposals that offer a diversity of viewpoints, rather than multiple examples of a single perspective. For those proposals that are not selected for publication, the authors will have an opportunity to post the abstract or full comment on a future Perspectives discussion forum.

\section{Acknowledgment}

This article is based on the James McKeen Cattell Award Address of the same title presented at the 22nd Annual Meeting of the Association of Psychological Science, Boston, MA.

\section{Declaration of Conflicting Interests}

The authors declared that they had no conflicts of interest with respect to their authorship or the publication of this article.

\section{Notes}

1. We use the term mental illness rather than a new term (e.g., burden of psychological dysfunction) to connect to the broader literatures in which the burden of mental and physical disability has been evaluated. Also, our discussion focuses on the burden of mental illness within the United States, although of course the burden is worldwide.

2. Psychotherapy is defined broadly in this article to include systematic efforts to apply psychosocial intervention to reduce distress or maladaptive behavior or enhance adaptive functioning. Psychosocial intervention, in contrast to medical or biological intervention (e.g., medication, surgery), focuses on such means of change as interpersonal interaction and systematic experiences (e.g., new ways of behaving through practice, role-playing, homework assignments, advice) designed to produce change. The therapist provides conditions to alleviate that person's distress and to improve functioning in everyday life (e.g., Garfield, 1980; Mahrer, 1986). The interaction is designed to alter the feelings, thoughts, attitudes, or actions of the person who has sought or has been brought to treatment.

3. CONSORT encompasses multiple procedures to improve the quality of reporting of empirical tests (www.consort-statement.org/). Examples include detailed information about participant inclusion criteria, recruitment, screening, and attrition; how the intervention was administered; and unplanned changes from the protocol. The standards have been adopted by hundreds of professional journals from many disciplines and countries (see www.consort-statement. org/about-consort/supporters/consort-endorsers-journals/).

4. Understandably, there is professional skepticism about self-help books and materials, given an unrelenting tsunami of such products based on opinion, clinical (but not empirical) evidence, and both common and uncommon sense. It is true that the majority of self-help materials in the local bookstore have not been evaluated empirically. In fairness, it is appropriate to note as well that the vast majority of psychotherapies in use have no supporting evidence for their effectiveness (see Kazdin, 2000).

5. The enacted laws are referred to as the Patient Protection and Affordable Care Act (P.L. 111-148) and the Health Care and Education Reconciliation Act of 2010 (P.L. 111-152; see http:// frwebgate.access.gpo.gov/cgi-bin/getdoc.cgi?dbname=111_cong_ bills\&docid=f:h3590eas.txt.pdf; http://frwebgate.access.gpo.gov/ cgi-bin/getdoc.cgi?dbname=111_cong_bills\&docid=f:h4872enr. txt.pdf)

\section{References}

Alegría, M., Canino, G., Shrout, P.E., Woo, M., Duan, N., Vila, D., et al. (2008). Prevalence of mental illness in immigrant and nonimmigrant U.S. Latino groups. American Journal of Psychiatry, 165, 359-369.

American Psychiatric Association. (2000). Diagnostic and statistical manual of mental disorders (4th ed., text rev.). Washington, DC: American Psychological Association. 
Amminger, G.P., Schäfer, M.R., Papageorgiou, K., Klier, C.M., Cotton, S.M., Harrigan, S.M., et al. (2010). Long-chain $\omega-3$ fatty acids for indicated prevention of psychotic disorders: A randomized, placebo-controlled trial. Archives of General Psychiatry, 67, 146-154.

Barak, A., Hen, L., Boniel-Nissim, M., \& Shapira, N. (2008). A comprehensive review and a meta-analysis of the effectiveness of Internet-based psychotherapeutic interventions. Journal of Technology in Human Services, 26, 109-160.

Bauer, S., Percevic, R., Okon, E., Meermann, R., \& Kordy, H. (2003). Use of text messaging in the aftercare of patients with bulimia nervosa. European Eating Disorders Review, 11, 279-290.

Berglund, B., Lindvall, T., \& Schwela, D.H. (Eds.). (1999). Guidelines for community noise. Geneva, Switzerland: World Health Organization. Retrieved from www.who.int/docstore/peh/noise/ guidelines 2.html

Bickman, L. (2008). A measurement feedback system (MFS) is necessary to improve mental health outcomes. Journal of the American Academy of Child and Adolescent Psychiatry, 47, 1114-1119.

Boschen, M.J. (2009a). Mobile telephones and psychotherapy: I. Capability and applicability. Behavior Therapist, 32, 168-175.

Boschen, M.J. (2009b). Mobile telephones and psychotherapy: II. A review of empirical research. Behavior Therapist, 32, 175-181.

Bouchard, M.F., Bellinger, D.C., Weuve, J., Matthews-Bellinger, J., Gilman, S.E., Wright, R.O., et al. (2009). Blood lead levels and major depressive disorder, panic disorder, and generalized anxiety disorder in U.S. young adults. Archives of General Psychiatry, 66, 1313-1319.

Bouchard, M.F., Bellinger, D.C., Wright, R.O., \& Weisskopf, M.G. (2010). Attention-deficit/hyperactivity disorder and urinary metabolites of organophosphate pesticides. Pediatrics, 125, e1270-e1277.

Cacioppo, J.T. (2007, October). The rise in collaborative psychological science. Observer, 20(9), 1-3. Retrieved from www. psychologicalscience.org/observer/getArticle.cfm?id=2228

Charles, D. (2009). Soap operas to save energy. Science, 325, 807.

Chorpita, B.F., Bernstein, A.D., Daleiden, E.L., \& The Research Network on Youth Mental Health. (2008). Driving with roadmaps and dashboards: Using information resources to structure the decision models in service organizations. Administration and Policy in Mental Health and Mental Health Services Research, 35, 114-123.

Cohen, J. (1988). Statistical power analysis in the behavioral sciences (2nd ed.). Hillsdale, NJ: Erlbaum.

Commission on Ethnic Minority Recruitment, Retention, and Training. (2008). Progress report. Washington, DC: American Psychological Association.

Crosby, R., DiClemente, R.J., Charnigo, R., Snow, G., \& Troutman, A. (2009). A brief, clinic-based, safer sex intervention for heterosexual African American men newly diagnosed with an STD: A randomized controlled trial. American Journal of Public Health, 99, S96-S103.

Cummins, S.E., Bailey, L., Campbell, S., Koon-Kirby, C., \& Zhu, S. (2007). Tobacco quitlines in North America: A descriptive study. Tobacco Control, 16, i9-i15.

Davison, K.P., Pennebaker, J.W., \& Dickerson, S.S. (2000). Who talks? The social psychology of illness support groups. American Psychologist, 55, 205-217.
Dickerson, F.B., \& Lehman, A.F. (2006). Evidence-based psychotherapy for schizophrenia. Journal of Nervous and Mental Disease, 194, 3-9.

Dimeff, L.A., Paves, A.P., Skutch, J.M., \& Woodcock, E.A. (2011). Shifting paradigms in clinical psychology: How innovative technologies are shaping treatment delivery. In D.H. Barlow (Ed.), The Oxford handbook of clinical psychology (pp. 618-648). New York: Oxford University Press.

Druss, B.G., Hwang, I., Petukhova, M., Sampson, N.A., Wang, P.S., \& Kessler, R.C. (2009). Impairment in role functioning in mental and chronic medical disorders in the U.S.: Results from the National Comorbidity Survey Replication. Molecular Psychiatry, 14, 728-737.

Gabbard, G.O., Lazar, S.G., Hornberger, J., \& Spiegel, D. (1997). The economic impact of psychotherapy: A review. American Journal of Psychiatry, 154, 147-155.

Garfield, S.L. (1980). Psychotherapy: An eclectic approach. New York: Wiley.

Greenberg, P.E., Sisitsky, T., Kessler, R.C., Finkelstein, S.N., Berndt, E.R., Davidson, J.R., et al. (1999). The economic burden of anxiety disorders in the 1990s. Journal of Clinical Psychiatry, $60,427-435$.

Griner, D., \& Smith, T.B. (2006). Culturally adapted mental health intervention: A meta-analytic review. Psychotherapy: Theory, Research, Practice, Training, 43, 531-548.

Harwood, H., Fountain, D., \& Livermore, G. (1998). The economic costs of alcohol and drug abuse in the United States: 1992. Rockville, MD: National Institute on Drug Abuse and the National Institute on Alcohol Abuse and Alcoholism.

Harwood, T.M., \& L'Abate, L. (2010). Self-help in mental health: A critical review. New York: Springer.

Hasler, B.P., Mehl, M.R., Bootzin, R.R., \& Vazire, S. (2008). Preliminary evidence of diurnal rhythms in everyday behaviors associated with positive affect. Journal of Research in Personality, 42, 1537-1546.

Health Resources and Services Administration. (2010). Health professional shortage areas: Mental health designated populations. Rural Assistance Center. Retrieved from www.raconline.org/ maps/mapfiles/hpsa_mental.png

Hiller, F.S., \& Leiberman, G.J. (2005). Introduction to operations research (9th ed.). New York: McGraw Hill.

Hinrichsen, G.A. (2010). Public policy and the provision of psychological services to older adults. Professional Psychology: Research and Practice, 41, 97-103.

Hoagwood, K.E., Cavaleri, M.A., Olin, S.S., Burns, B.J., Slaton, E., Gruttadaro, D., \& Hughes, R. (2010). Family support in children's mental health: A review and synthesis. Clinical Child and Family Psychology Review, 13, 1-45.

Hoge, M.A., Morris, J.A., Daniels, A.S., Stuart, G.W., Huey, L.Y., \& Adams, N. (2007). An action plan for behavioral health workforce development. Washington, DC: Department of Health and Human Services.

Hughes, J.P., Garnett, G.P., \& Koutsky, L. (2002). The theoretical population-level impact of a prophylactic human papilloma virus vaccine. Epidemiology, 13, 631-639. 
Institute of Medicine. (2008). Retooling for an aging America: Building the health care workforce. Washington, DC: National Academies Press.

Jason, L.A., \& Ferrari, J.R. (2010). Oxford House recovery homes: Characteristics and effectiveness. Psychological Services, 7, 92-102.

Joy, C.B., Mumby-Croft, R., \& Joy, L.A. (2003). Polyunsaturated fatty acid supplementation for schizophrenia. Cochrane Database Systematic Reviews, CD001257.

Kaplan, B.J., Crawford, S.G., Field, C.J., \& Simpson, J.S.A. (2007). Vitamins, minerals, and mood. Psychological Bulletin, 133, 747-760.

Kaplan, E.H., Craft, D.L., \& Wein, L.M. (2002). Emergency response to a smallpox attack: The case for mass vaccination. Proceedings of the National Academy of Sciences, USA, 99, 10935-10940.

Kazdin, A.E. (2000). Psychotherapy for children and adolescents: Directions for research and practice. New York: Oxford University Press.

Kazdin, A.E. (2007). Mediators and mechanisms of change in psychotherapy research. Annual Review of Clinical Psychology, 3, 1-27.

Kessler, R.C., Aguilar-Gaxiola, S., Alonso, J., Chatterji, S., Lee, S., Ormel, J., et al. (2009). The global burden of mental disorders: An update from the WHO World Mental Health (WMH) Surveys. Epidemiologia e Psichiatria Sociale, 18, 23-33.

Kessler, R.C., Akiska, H.S., Ames, M., Birnbaum, H., Greenberg, P., Hirschfeld, R.M., et al. (2006). Prevalence and effects of mood disorders on work performance in a nationally representative sample of U.S. workers. American Journal of Psychiatry, 163, 1561-1568.

Kessler, R.C., Demler, O., Frank, R.G., Olfson, M., Pincus, H.A., Walters, E.E., et al. (2005). Prevalence and treatment of mental disorders, 1990 to 2003. New England Journal of Medicine, 352, 2515-2523.

Kessler, R.C., Heeringa, S., Lakoma, M.D., Petukhova, M., Rupp, A.E., Schoenbaum, M., et al. (2008). Individual and societal effects of mental disorders on earnings in the United States: Results from the National Comorbidity Survey Replication. American Journal of Psychiatry, 165, 703-711.

Kessler, R.C., \& Wang, P.S. (2008). The descriptive epidemiology of commonly occurring mental disorders in the United States. Annual Review of Public Health, 29, 115-129.

Kliegl, R. (2008). Publication statistics show collaboration, not competition. Observer, 21(6):29-31.

Krupnick, J.L., \& Pincus, H.A. (1992). The cost-effectiveness of psychotherapy: A plan for research. American Journal of Psychiatry, 149, 1295-1305.

Lichtenstein, E., Zhu, S., \& Tedeschi, G.J. (2010). Smoking cessation quitlines: An underrecognized intervention success story. American Psychologist, 65, 252-261.

Magal, P., \& Ruan, S. (Eds.). (2008). Structured population models in biology and epidemiology. Heidelberg, Germany: Springer-Verlag.

Maher, J.E., Rohde, K., Dent, C.W., Stark, M.J., Pizacani, B., Boysun, M.J., et al. (2007). Is a statewide tobacco quitline an appropriate service for specific populations? Tobacco Control, 16, i65-i70.

Mahrer, A. (1986). The goals of psychotherapy. New York: Appleton-Century-Crofts.
Merikangas, K.R., Ames, M., Cui, L., Stang, P.E., Ustun, T.B., Von Korff, M., \& Kessler, R.C. (2007). The impact of comorbidity of mental and physical conditions on role disability in the U.S. adult household population. Archives of General Psychiatry, 64, 1180-1188.

Miranda, J., Bernal, G., Lau, A.S., Kohn, L., Hwang, W.C., \& LaFromboise, T. (2005). State of the science on psychosocial interventions for ethnic minorities. Annual Review of Clinical Psychology, 1, 113-143.

Moher, D., Schulz, K.F., \& Altman, D. (2001). The CONSORT statement: Revised recommendations for improving the quality of reports of parallel-group randomized trials. Journal of the American Medical Association, 285, 1987-1991.

Mohr, D.C., Vella, L., Hart, S., Heckman, T., \& Simon, G. (2008). The effect of telephone-administered psychotherapy on symptoms of depression and attrition: A meta-analysis. Clinical Psychology: Science and Practice, 15, 243-253.

Morris, M.E., Kathawala, Q., Leen, T.K., Gorenstein, E.E., Guilak, F., Labhard, M., \& Deleeuw, W. (2010). Mobile therapy: Case study evaluations of a cell phone application for emotional self-awareness. Journal of Medical Internet Research, 12, e10.

Muñoz, R.F., Lenert, L.L., Delucchi, K., Stoddard, J., Perez, J.E., Penilla, C., \& Pérez-Stable, E.J. (2006). Toward evidence-based Internet interventions: A Spanish/English Web site for international smoking cessation trials. Nicotine \& Tobacco Research, 8, 77-87.

Murray, C.J.L., \& Frenk, J. (2008). Health metrics and evaluation: Strengthening the science. Lancet, 371, 1191-1199.

Nathan, P.E., \& Gorman, J.M. (Eds.). (2007). A guide to treatments that work (3rd ed.). New York: Oxford University Press.

Newman, F.L., \& Howard, K.I. (1986). Therapeutic effort, treatment outcome, and national health policy. American Psychologist, 41, 181-187.

Newman, M.G. (2000). Recommendations for a cost-offset model of psychotherapy allocation using generalized disorder as an example. Journal of Consulting and Clinical Psychology, 68, 549-555.

Pentland, A. (2008). Honest signals: How they shape our world. Cambridge, MA: MIT Press.

Reacher, M., McKenzie, K., Lane, C., Nichols, T., Kedge, I., Iversen, A., et al. (2004). Health impacts of flooding in Lewes: A comparison of reported gastrointestinal and other illness and mental health in flooded and non-flooded households, Communicable Disease and Public Health, 7, 39-46.

Rice, V.H., \& Stead, L.F. (2008). Nursing interventions for smoking cessation. Retrieved from http://info.onlinelibrary.wiley. com/userfiles/ccoch/file/World\%20No\%20Tobacco\%20Day/CD00 1188.pdf

Richards, J.C., \& Alvarenga, M.E. (2002). Extension and replication of an Internet-based treatment program for panic disorder. Cognitive Behaviour Therapy, 31, 41-47.

Richards, J.M., \& Gottfredson, G.D. (1978). Geographic distribution of U.S. psychologists: A human ecological analysis. American Psychologist, 33, 1-9.

Ritterband, L.M., Gonder-Frederick, L.A., Cox, D.J., Clifton, A.D., West, R.W., \& Borowitz, S.M. (2003). Internet interventions: In review, in use, and into the future. Professional Psychology: Research and Practice, 34, 527-534. 
Rochlin, A.B., Zack, J.S., \& Speyer, C. (2004). Online therapy: Review of relevant definitions, debates, and current empirical support. Journal of Clinical Psychology, 60, 269-283.

Sanders, M.R. (2008). Triple P-Positive Parenting Program as a public health approach to strengthening parenting. Journal of Family Psychology, 22, 506-517.

Sanders, M.R., \& Murphy-Brennan, M. (2010). The international dissemination of the Triple P-Positive Parenting Program. In J.R. Weisz \& A.E. Kazdin (Eds.). Evidence-based psychotherapies for children and adolescents (2nd ed., pp. 519-537). New York: Guilford Press.

Satcher, D., Friel, S., \& Bell, R. (2007). Natural and manmade disasters and mental health. Journal of the American Medical Association, 298, 2540-2542.

Sher, T.G., \& Halford, W.K. (Eds.). (2008). Special section: Public health perspectives on family interventions. Journal of Family Psychology, 22, 495-528.

Singhal, A., Cody, M.J., Rogers, E.M., \& Sabido, M. (Eds.). (2003). Entertainment-education and social change: History, research, and practice. Mahwah, NJ: Erlbaum.

Singhal, A., \& Rogers, E.M. (1999). Entertainment-education: A communication strategy for social change. Mahwah, NJ: Erlbaum.

Stead, L.F., Bergson, G., \& Lancaster, T. (2008). Physician advice for smoking cessation. Retrieved from http://info.onlinelibrary.wiley. com/userfiles/ccoch/file/World\%20No\%20Tobacco\%20Day/CD 000165.pdf

Swartz Woods, S., \& Haskins, A.E. (2007). Increasing reach of quitline services in a U.S. state with comprehensive tobacco treatment. Tobacco Control, 16, i33-i36.

Turrisi, R., Jaccard, J., Taki, R., Dunnam, H., \& Grimes, J. (2001). Examination of the short-term efficacy of a parent intervention to reduce college student drinking tendencies. Psychology of Addictive Behaviors, 15, 366-372.
Ulmer, C., Bruno, M., \& Burke, S. (Eds.). (2010). Future directions for the National Healthcare Quality and Disparities Reports. Washington, DC: National Academies Press.

Weissman, M.M., Markowitz, J.C., \& Klerman, G.L. (2000). Comprehensive guide to interpersonal psychotherapy. New York: Basic Books.

Weisz, J.R., \& Kazdin, A.E. (Eds.). (2010). Evidence-based psychotherapies for children and adolescents (2nd ed.). New York: Guilford Press.

Wells, K., Klap, R., Koike, A., \& Sherbourne, C. (2001). Ethnic disparities in unmet need for alcoholism, drug abuse, and mental health care. American Journal of Psychiatry, 158, 2027-2032.

World Health Organization. (2007). International statistical classification of diseases and related health problems (10th rev.). Retrieved from http://apps.who.int/classifications/apps/icd/ icd10online/

Wuchty, S.W., Jones, B., \& Uzzi, B. (2007). The increasing dominance of teams in the production of knowledge. Science, 316, 1036-1039.

Yates, B.T. (1995). Cost-effectiveness analysis, cost-benefit analysis, and beyond: Evolving models for the scientist-managerpractitioner. Clinical Psychology: Science and Practice, 2, 385-398.

Ybarra, M.L., \& Eaton, W.W. (2005). Internet-based mental health interventions. Mental Health Services Research, 7, 75-87.

Zhu, S.-H., \& Pierce, J.P. (1995). A new scheduling method for time-limited counseling. Professional Psychology: Research and Practice, 26, 624-625.

Zhu, S.-H., Wong, S., Stevens, C., Nakashima, D., \& Gamst, A. (2010). Use of a smokers' quitline by Asian language speakers: Results from 15 years of operation in California. American Journal of Public Health, 100, 846-852. 


\title{
Reactions to the Call to Reboot Psychotherapy Research and Practice: Introduction to Special Section of Comments on Kazdin and Blase (20II)
}

Perspectives on Psychological Science 6(5) 475-477

(C) The Author(s) 2011

Reprints and permission:

sagepub.com/journalsPermissions.nav DOI: $10.1177 / 1745691611418242$

http://pps.sagepub.com

@SAGE

\author{
Bethany A.Teachman' and Teresa A. Treat ${ }^{2}$ \\ 'Department of Psychology, University of Virginia, Charlottesville, and ${ }^{2}$ Department of Psychology, \\ University of lowa, lowa City
}

\section{Keywords}

mental illness, intervention, psychotherapy, dissemination, service delivery

The field of clinical science has made great progress in developing evidence-based treatments, but we have failed to reach our fundamental goal of reducing the prevalence of mental illness in society and its devastating effects on psychosocial functioning on a large scale (Baker, McFall, \& Shoham, 2008). We can often help a given individual who is struggling with mental illness, but how are we to stem the tide of human suffering?

In "Rebooting Psychotherapy Research and Practice to Reduce the Burden of Mental Illness," Alan Kazdin and Stacey Blase (2011b) argued that individual psychotherapy, the dominant method used to deliver treatment, cannot possibly meet society's overwhelming mental health needs. The rates of mental illness are too high; approximately half of all Americans will meet diagnostic criteria for at least one psychiatric disorder during their lifetime (Kessler \& Wang, 2008), yet over half of these individuals will not receive treatment (Kessler et al., 2005). Given the variability in the reasons people do not receive professional help (e.g., lack of access to a trained clinician, stigma, cultural obstacles, limited resources), a one-size-fits-all model of treatment delivery is not now, nor will it ever be, effective. Kazdin and Blase thus advocate for development of a portfolio of treatment delivery methods, including harnessing available technologies, such as web- and phone-based interventions, providing treatment in everyday settings (rather than exclusively in the therapist's office), using nontraditional providers of interventions, promoting self-help approaches, and using the media to communicate prevention and intervention messages to large segments of the population.

Kazdin and Blase call for radical reform in how we think about delivering prevention and intervention approaches. They note that it will be critical to identify the mechanisms of change underlying successful interventions to determine how they can be abbreviated for broader dissemination without losing their essential ingredients. Also, they outline the need for a national database that can be used to establish a baseline and reference point for examining change in the burden of mental illness over time, as well as the key predictors and moderators of burden reduction. Although they acknowledge that a shift toward a broad portfolio of delivery models will be controversial and raise new challenges for traditional mental health providers, they also argue that the only way to effect widespread change is to connect with other disciplines outside of standard practice (e.g., in epidemiology, nutrition, math modeling, technology development, etc.) and to dramatically alter what we construe as being under the exclusive purview of therapists.

This call to action would demand change in our research, service delivery, grant funding and training models. Recognizing that calling for such a dramatic shift in the field would elicit strong responses, Perspectives on Psychological Science put out an open call for comments on Kazdin and Blase's proposal. The caliber and diversity of the 26 submissions made it quite challenging to select only 6 to include in this issue. (Additional comments can be submitted and viewed in the online version of the original article at http://pps.sagepub.com/content/6/1/21. full; click on "Read all comments" under the Reader Responses sidebar, then click "Full Text.") Submissions were rated for likely impact, intellectual rigor, originality, and overall quality. The final selection of comments offers a diverse set of perspectives on how we can most effectively shift toward reducing the global burden of mental illness.

Varda Shoham and Thomas Insel (2011, this issue) provide a unique perspective on the Kazdin and Blase article, writing in their roles as the Director (Insel) and Special Assistant to the Director, Division of Adult Translational Research and

\section{Corresponding Author:}

Bethany A. Teachman, Department of Psychology, University of Virginia, P.O. Box 400400, Charlottesville,VA 22904-4400

E-mail: bteachman@virginia.edu 
Treatment Development (Shoham) of the National Institute of Mental Health (NIMH). They argue that reducing the burden of mental illness requires more consistently and carefully considering for whom simplified intervention and prevention approaches will be sufficient and for whom more extensive approaches will be needed. To achieve this goal of determining what type and level of intervention are needed for a given individual, they outline the importance of Attribute by Treatment Interaction (ATI) research, which examines how a given individual characteristic (e.g., baseline symptom severity, age of illness onset) interacts with the treatment conditions to predict treatment response. They review stories of success and failure in ATI research, highlighting initiatives from NIMH that can help support ATI research to advance more personalized prevention and intervention work.

Marc Atkins and Stacey Frazier (2011, this issue) suggest that the time is ripe for adoption of a multilevel model of care that subsumes universal prevention strategies, targeted efforts with high-risk populations, and more individualized management of persons with intensive needs. Comprehensive integration of prevention and intervention services across these three levels ideally would extend the reach of mental health services while decreasing the number of individuals needing more costly and time-intensive individualized treatment. Atkins and Frazier stress that the success of this public health approach would necessitate investing to a much greater degree in the training and support of nontraditional providers in nontraditional settings (e.g., laypersons in natural community settings, personnel in social-services agencies). They note how their proposal is consistent with the goals of recently enacted health-care reform in this country, as well as recommendations from the World Health Organization.

Bruce Chorpita and colleagues (2011, this issue) argue that the field has overemphasized knowledge production to the detriment of knowledge management. In particular, they argue that whereas the focus on developing new evidence-based treatments has led to many positive advances in clinical care, it has also created an untenable situation. There are so many separate treatment manuals that there is no way that a given psychologist can possibly learn even a fraction of them, or know how to combine them for clients with comorbidity. Instead, Chorpita and colleagues propose a shift in emphasis to focus on knowledge management: novel approaches to develop, administer, and organize interventions. They outline a variety of strategies that can be used to aggregate our existing knowledge, such as discerning the essential "practice elements" to treat a given set of symptoms by looking at which clinical procedures are commonly associated with good treatment outcomes across clinical trials.

Idit Shalev and John Bargh (2011, this issue) share an innovative proposal to leverage priming strategies developed by experimental social psychologists for the modification of nonconscious processes that perpetuate maladaptive behavior. They describe how a variety of simple visual or physical experiences (e.g., inducing feelings of physical warmth) could be used to promote feelings of social warmth, goals of emotion regulation, or adherence to treatment. To date, such methods have been used only to modify nonclinical behavior in research contexts. Shalev and Bargh suggest that variants of these techniques potentially could be individualized, presented via contemporary technological devices, and deployed widely at low cost to set the stage for clinically relevant change.

Brian Yates (2011, this issue) makes the case that we must do more to investigate different modes of delivering treatments, so that we can reduce costs and reach more people. He advocates for funding to evaluate different delivery methods (rather than just different treatments) and challenges researchers to make delivery costs more explicit in their calculations of cost effectiveness. Yates cites numerous examples of delivery systems that have the potential to reduce resource use and costs, yet still pass on most (if not all) of a given treatment's effectiveness, including Internet-based interventions, automated phone interventions, and video-based interventions. He argues that we know a lot about the ingredients (treatment approaches) needed to help people who are suffering, but we need to study the "spoon" (delivery method) that passes these ingredients along to the hungry client: "Just as therapy is no longer an art but a science based on research evidence gathered in clinical settings, so too can be its delivery" (p. 498).

Denise Sloan, Brian Marx, and Terence Keane describe a plethora of recent service-delivery innovations launched by the Veterans Health Administration (VA) to enhance the mental health care provided to the almost 2 million veterans in this country (2011, this issue). First, the VA is leveraging technological resources such as the Internet, smartphones, and videoconferencing to expand the reach and availability of potentially anonymous services. Second, the VA is investing in the training and support of laypersons for the provision of mental health care, including VA chaplains, VA police, and peers. Third, the VA has committed to systemwide dissemination and implementation of evidence-based treatments for mentalhealth problems, necessitating the development and evaluation of large-scale training, consultation, and monitoring strategies. The VA's efforts provide excellent models for the comprehensive dissemination and implementation of such treatments on a nationwide scale.

Finally, the special section concludes with an incisive reply from Kazdin and Blase (2011a, this issue). As evidenced both by their provocative, original article and by the strong response it has already elicited in the field, change is afoot in intervention science. These commentaries flesh out many of Kazdin and Blase's suggestions and highlight the diverse means by which these issues are being tackled. Kazdin and Blase provide an exciting forum for conceptualizing, implementing, and disseminating strategies aimed at reducing the burden of mental illness. The current special section continues this important conversation.

\section{Declaration of Conflicting Interests}

The author(s)declared no potential conflicts of interest with respect to the research, authorship, and/or publication of this article. 


\section{Funding}

The author(s) received no financial support for the research, authorship, and/or publication of this article.

\section{References}

Atkins, M., \& Frazier, S. (2011). Expanding the toolkit or changing the paradigm: Are we ready for a public health approach to mental health? Perspectives on Psychological Science, 6, 483-487.

Baker, T. B., McFall, R. M., \& Shoham, V. (2008). Current status and future prospects of clinical psychology: Toward a scientifically principled approach to mental and behavioral health care. Psychological Science in the Public Interest, 9, 67-103.

Chorpita, B., Rotheram-Borus, M. J., Daleiden, E., Bernstein, A., Cromley, T., Swendeman, D., \& Regan, J. (2011). The old solutions are the new problem: How do we better use what we already know about reducing the burden of mental illness? Perspectives on Psychological Science, 6, 493-497.

Kazdin, A. E., \& Blase, S. L. (2011a). Interventions and models of their delivery to reduce the burden of mental illness: Reply to commentaries. Perspectives on Psychological Science, 6, $507-510$.
Kazdin, A.E., \& Blase, S. L. (2011b). Rebooting psychotherapy research and practice to reduce the burden of mental illness. Perspectives on Psychological Science, 6, 21-37.

Kessler, R. C., Demler, O., Frank, R. G., Olfson, M., Pincus, H. A., Walters, E. E., . . Zaslavsky, A. M. (2005). Prevalence and treatment of mental disorders, 1990 to 2003. New England Journal of Medicine, 352, 2515-2523.

Kessler, R. C., \& Wang, P. S. (2008). The descriptive epidemiology of commonly occurring mental disorders in the United States. Annual Review of Public Health, 29, 115-129.

Shalev, I., \& Bargh, J. (2011). Use of priming-based interventions to facilitate psychological health. Commentary on Kazdin and Blase (2011). Perspectives on Psychological Science, 6, 488-492.

Shoham, V., \& Insel, T. R. (2011). Rebooting for whom? Portfolios, technology, and personalized intervention. Perspectives on Psychological Science, 6, 478-482.

Sloan, D. M., Marks, B. P., \& Keane, T. M. (2011). Reducing the burden of mental illness in military veterans: Commentary on Kazdin and Blase (2011). Perspectives on Psychological Science, 6, 503-506.

Yates, B. (2011). Delivery systems can determine therapy costs and effectiveness, more than type of therapy. Perspectives on Psychological Science, 6, 498-502. 


\title{
Rebooting for Whom? Portfolios, Technology, and Personalized Intervention
}

Perspectives on Psychological Science 6(5) $478-482$

(C) The Author(s) 2011

Reprints and permission:

sagepub.com/journalsPermissions.nav DOI: $10.1177 / 1745691611418526$

http://pps.sagepub.com

@SAGE

\author{
Varda Shoham ${ }^{1,2}$ and Thomas R. Insel ${ }^{2}$ \\ 'University of Arizona, Tucson, and ${ }^{2}$ National Institute of Mental Health, Bethesda, MD
}

\begin{abstract}
We share Kazdin and Blase's (20II) sense of urgency about finding better ways to reduce the burden of mental illness. Although effective psychosocial treatments exist, they do not often reach the patients who need them most. Kazdin and Blase's portfolio approach aims to cast a wider net through increased use of technology, media, self-help, nonprofessional providers, and collaborations with other disciplines. It is unclear, however, whether reaching more people would suffice to reduce the burden of mental illness, much less offset the small effect sizes of simplified, scaled-down interventions such a portfolio approach would likely entail.We focus here on an underdeveloped theme in Kazdin and Blase's essay-that bending the curve of mental illness will require better knowledge of for whom simplified intervention and prevention strategies will suffice and for whom more intensive intervention is necessary. Such "for whom" questions deserve a central place on the national research agenda as we move toward individualized or personalized health care. In the absence of such knowledge, we risk treatment decisions guided by accessibility to resources rather than patient needs-the very problem Kazdin and Blase aim to solve.
\end{abstract}

\section{Keywords}

treatment, mental illness, personalized intervention

We share Kazdin and Blase's (2011) sense of urgency about finding better ways to reduce the burden of mental illness. Although effective psychosocial treatments exist, they often do not reach the patients who need them most. Kazdin and Blase's portfolio approach aims to cast a wider net of empirically supported interventions through increased use of technology, media, self-help, nonprofessional providers, and collaborations with other disciplines. The authors' off-theshelf examples provide an intriguing glimpse of what this could look like and usefully bridge the artificial boundary between prevention and treatment. Because efficacious treatments will usually require simplification for portfolio dissemination, we especially appreciate Kazdin and Blase's cautionary note about the importance of understanding how these treatments work. Without such knowledge, an abbreviated (though more accessible) intervention could unwittingly sacrifice essential mechanisms of change.

Although reaching more people is a laudable aim, it is not clear whether this by itself will reduce the burden of mental illness, much less offset the small effect sizes of simplified, scaleddown interventions such a portfolio approach would likely entail. It is interesting that the same National Comorbidity Study the authors cite for burden statistics demonstrated a $50 \%$ increase in treatment between 1991 and 2001 without any decrease in prevalence or morbidity (Kessler, Berglunk, Borges, Nock, \&Wang, 2005; Kessler et al., 2005). Apparently, more treatment does not necessarily mean less burden, especially if the treatment is insufficient or inappropriate.

In this commentary, we focus mainly on an underdeveloped theme in Kazdin \& Blase's essay - that bending the curve of mental illness will require better knowledge of for whom simplified intervention and prevention strategies will suffice and for whom more intensive intervention is necessary. In our view such "for whom" questions deserve a more central place on the national research agenda as we move toward individualized or personalized health care. In the absence of such knowledge, we risk treatment decisions guided by accessibility to resources rather than patient needs - the very problem Kazdin and Blase aim to solve.

\section{Which Treatments for Whom?}

As the cornerstone of personalized intervention, research on prospective treatment moderators (what works for whom) necessarily cuts across a wide range of case and treatment characteristics. The basic question in this Attribute $\times$ Treatment Interaction (ATI) paradigm is which cases characteristics

\section{Corresponding Author:}

Varda Shoham, Department of Psychology, University of Arizona, I503 E. University Boulevard, Tucson, AZ 8572I

E-mail:varda@email.arizona.edu 
moderate (interact with) which treatment conditions to predict clinical outcomes. Because the most conspicuous case-level moderators - psychiatric diagnosis - have not proved terribly useful for guiding psychosocial intervention, the search for meaningful moderators has recently expanded to include such diverse factors as current and historical problem severity, cognitive processes, and characteristics of the family social environment. There is equal or greater diversity on the treatment side, in which Kazdin and Blase's big-menu portfolio approach now ups the ante on answering "for whom" questions to guide selection of interventions. And this is not to mention an evenmore pressing ATI question where serious mental illness is concerned-namely, for whom will combined psychosocial intervention and pharmacotherapy be more beneficial than either modality alone (Nemeroff et al., 2003).

Psychosocial ATI research is not new, dating back at least to Cronbach and Snow (1977), and its history has included both excitement and disappointments. For example, the hope engendered by early ATI findings in psychotherapy research (e.g., Beutler, 1991; Shoham-Salomon \& Hannah, 1991) was dampened by the high-profile failure of Project Match Research Group (1997) to find significant moderators of alcoholism treatments. Factors limiting the yield of ATI research include investigation of post hoc, hard-to-replicate moderators; moderators unrelated to theory-derived, hypothesized mechanisms of change; underpowered tests of moderation; comparisons among similar or overlapping treatments (as in Project MATCH), which limits moderator detection; and unbalanced measurement of $A$ and $T$ variables, in which researchers assess case attributes $(A \mathrm{~s})$ in precise detail while documenting treatments (and treatment fidelity) only grossly. Shulman (1981) noted years ago this tendency to measure $A \mathrm{~s}$ with micrometers and $T \mathrm{~s}$ with divining rods.

Despite this checkered history, recent years have seen a burgeoning interest in treatment moderators, and preliminary findings highlight the importance of pursuing this line of research more vigorously than we have so far. Thus, regarding cognitive treatments for depression, there is good evidence that optimal treatment selection depends on factors such as age of illness onset (Jarrett et al., 2001), current and historical symptom severity (Bockting et al., 2005; Fournier et al., 2011), and patient preference for pharmacological or psychosocial intervention (Kocsis et al., 2009). Similarly, in the schizophrenia domain, a patient's age at treatment initiation appears to moderate the effects of cognitive rehabilitation (CR) interventions, such that younger patients $(<40)$ benefit more than older patients from receiving CR (McGurk \& Mueser, 2008; Wykes et al., 2009).

A more promising class of potential treatment moderators relates to theory-derived mechanisms of problem formation or problem maintenance. For example, there is evidence that baseline levels of maladaptive cognitions hypothesized to maintain depressive symptoms serve to moderate the success of cognitive-behavior therapies relative to control conditions (Hollon et al., 2005) and that theory-relevant aspects of a patient's trauma history are associated with better response to a variant of cognitive behavior therapy than to antidepressants (Nemeroff et al., 2003). Similarly, looking beyond the patient, the quality of family or couple communication appears to moderate the success of family- and couple-focused treatments (Miklowitz et al., 2009; Shoham, Rohrbaugh, Stickle, \& Jacob, 1998).

Despite these advances, we are still a long way from having a sufficient body of evidence to guide psychosocial variants of personalized medicine based on treatment-matching algorithms. Part of the problem is that current evidence based on statistical significance often lacks clinical significance. Our search for moderators is still very much in a discovery phase, and the clinical value of any discoveries will ultimately need to be tested in clinical trials with patients stratified on putative moderator variables.

Where can we expect to discover the most promising moderators? The horizon includes several promising developments we think are worth mentioning. One is the Research Domain Criteria (RDoC) project at the National Institute of Mental Health (NIMH; Insel \& Cuthbert, 2009; Sanislow et al., 2011), which is attempting to ground the patient attribute $(A)$ side of the ATI equation in underlying neurobiological dimensions of psychopathology. Given the high variability in pathophysiology among patients diagnosed with the same disorder (as determined by the Diagnostic and Statistical Manual of Mental Disorders), variability in treatment response among patients similarly classified is not surprising. The sciencebased, bottom-up $\mathrm{RDoC}$ approach to mental disorders aims to establish validity in ways that may ultimately align better with treatment response.

Other promising developments are methodological. For example, new applications of so-called adaptive randomized designs can illuminate the most efficient and effective sequencing of several interventions rather than just one - as when nonresponders to a first-line intervention receive more intensive treatment in a stepped-care framework (Collins, Dziak, \& Li, 2009; Collins, Murphy, \& Srecher, 2005). These adaptive designs are also well suited for testing treatment moderators, including for whom starting with a "light" version of a given treatment might ultimately prove iatrogenic or counterproductive. One could approach this question either sequentially, as in adaptive design research, or simultaneously, by considering moderators of pared-down interventions in the population more broadly.

A related innovation is the Kraemer, Wilson, Firburn, and Agras (2002) approach to creating moderator profiles. Whereas traditional ATI research employing group factorial designs typically stumbles on the prospect of multiple interacting moderators - Cronbach's (1975, p. 119) "hall of mirrors that extends to infinity" - the Kraemer et al. method allows for testing multiple moderators and identifying the strongest ones via a probability index of replication $\left(p_{\text {rep }}\right)$ instead of the traditional significance level. Thus, a $p_{\text {rep }} \leq .90$ indicates that there is at least a $90 \%$ chance to replicate the moderator's effect 
with a new sample from the same population, provided that the effect size of the moderator is at least as strong as the effect size of the treatment. This then leads to hypothesis-testing studies in which prospective, empirically based moderators serve as stratification variables.

In a notable application of this moderator-profile approach, Vittengl, Clark, and Jarrett (2010) investigated which responders require continuation-phase cognitive therapy (CT) to achieve stable remission of depressive symptoms and which could sustain positive treatment outcomes without further intervention. Based on examining a range of plausible and partially overlapping moderators in a hypothesis-generation framework, Vittengl et al. found that a profile including younger current age and younger age of onset in combination with high social inhibition and emotional detachment served to discriminate the criterion groups. They are now seeking to replicate this profile result in a prospective, hypothesis-testing design that could solidify an empirical basis for providing continuation CT. The investigators speculate that continuation CT may be "too little, too late" for some older patients, which brings us to the crucial role of "for whom" research in prevention.

\section{Prevention for Whom?}

Kazdin and Blase note that "the portfolio idea would be beneficial for conceptualizing the task of prevention because it begins with who ought to be reached in the population, what interventions are likely to accomplish that for various groups, and what the effects are" (p. 28). This important point deserves further development: On one hand, the potential payoffs from well-targeted preventive intervention could be enormous. On the other hand, without better understanding of who benefits from which prevention strategies, we risk shooting in the dark and hitting targets indiscriminately, which could be costly and even iatrogenic. Most important, we need to understand risk and resilience at an individual level. Despite some good leads on risk factors from both the nature (genetics) and nurture (experience) sides of the mental illness equation, we do not yet have biomarkers or psychological attributes with high predictability for any individual.

Like physical illnesses, most mental disorders have a clear developmental trajectory. It is disconcerting in this respect that treatment for mental disorders begins on average 11 years after problem onset (Wang et al., 2005). The field of medicine has rarely reduced the burden of any illness when initial intervention takes place so long after onset. Observable symptoms of mental illness, possibly reflecting underlying biological processes, may have a relatively long latency period. Add to this the long delay for treatment, and prospects for reducing the burden of mental illness appear even more daunting. At the risk of medicine envy, it is worth noting that early detection of specific risk factors coupled with "for whom" risk factor reduction interventions has enabled cardiology to realize a $60 \%$ reduction in mortality from coronary artery disease.
Imagine what just a fraction of that accomplishment could mean for mental health.

A promising line of prevention research involves early stage intervention with major mental illnesses such as schizophrenia and bipolar disorder. For example, a combination of features now allows detecting the prodrome of schizophrenia with more than $80 \%$ accuracy in adolescents who have not yet become psychotic (Cannon et al., 2008). Further along in the prevention spectrum, characteristics of the family environment moderate how adolescents with early-stage bipolar disorder respond to psychosocial interventions combined with medication; here a family-focused approach appears to be most beneficial — and perhaps essential — for families showing high "expressed emotion" (criticism, hostility, and emotional overinvolvement) in relation to the patient (Miklowitz et al., 2009).

This approach of personalized and preemptive interventions is a major focus of the NIMH Strategic Plan (www .nimh.nih.gov/about/strategic-planning-reports/index.shtml). Beyond studies of the prodrome of schizophrenia and bipolar disorder, we have launched a broad effort on biomarkers that could serve as moderators or predictors of response. One such study, EMBARC (which stands for Establishing Moderators/ Mediators for a Biosignature of Antidepressant Response in Clinical Care), is combining genomics, imaging, quantitative EEG, and cognitive measures to develop a profile or biosignature of antidepressant response. In another effort, the Study to Assess Risk and Resilience in Soldiers, we are looking for predictors of posttraumatic stress disorder and depression in soldiers. And in another, we are following younger siblings of children with autism to identify the earliest signs of this disorder. We hope that the identification of such risk factors will translate into treatment moderators, thus leading to better targeted interventions.

\section{Technology for Whom?}

Technology-assisted treatments are surely here to stay, but these too need better targeting to be efficient. Such interventions, sometimes referred to as e-Health (Baker, McFall, \& Shoham, 2008), are highly replicable and portable and thus easy to disseminate. Computer-based e-Health interventions have the additional virtue of permitting exposure to diverse realistic contexts achieved via virtual reality capabilities (Bordnick et al., 2008), and they have the potential to reduce utilization of more expensive health care options (Boberg et al., 1995). Because e-Health interventions allow for some tailoring based on a variety of patient characteristics (Strecher et al., 2005), we were surprised that Kazdin and Blase do not emphasize this.

Although technology could prove a game changer, it may also have some unintended consequences. As commentators like Abraham Verghese (2011) have pointed out, the complaints we hear from patients, family, and friends are rarely about the dearth of technology but about its excesses, turning 
patients into "i-patients." In the "which treatments for whom" framework of ATI research, we know virtually nothing about the treatments for which, or the patients for whom, a human relationship or therapeutic alliance is essential to productive behavior change. Without such knowledge, even in a best-case scenario, e-Health will to some extent require shooting in the dark. A worst-case scenario is that e-Health interventions could "spend out" some of our most effective techniques, rendering them less amenable to subsequent, face-to-face intervention.

It bears repeating that technology-based intervention portfolios imply simplifying and abbreviating treatments that were empirically, even experimentally, supported in their original format. Yet, by Kazdin's (2007) own account, the field does not know much (and certainly not enough) about how multicomponent or even simple psychosocial interventions actually work. Apart from the problem of abbreviated (if more accessible) interventions sacrificing essential mechanisms of change, we worry that pared-down portfolio interventions gaining premature adoption in community settings will yield effects no larger than those for "treatment as usual," which are very small. The e-Health picture may well improve as additional efficacy and effectiveness data accumulate, but in our view the "which treatment for whom" question will not soon go away.

On balance, Kazdin and Blase do the field an important service by highlighting the diverse ways in which technology could enhance the world of psychotherapy. We are hopeful that the increased access and increased flexibility of this approach will deliver improved outcomes. At the same time, we would caution that technology is a tool, not an answer: With a better understanding of how and for whom technologyassisted treatments work (see Amir, Taylor, \& Donohue, in press, for a promising example of this), mental health professionals should be in a better position to personalize psychosocial intervention and ultimately reduce the burden of mental illness.

\section{Declaration of Conflicting Interests}

The author(s)declared no potential conflicts of interest with respect to the research, authorship, and/or publication of this article.

\section{Funding}

The author(s) received no financial support for the research, authorship, and/or publication of this article.

\section{References}

Amir, N., Taylor, C. T., \& Donohue, M. C. (in press). Predictors of response to an attention modification program in generalized social phobia. Journal of Consulting and Clinical Psychology.

Baker, T., McFall, R., \& Shoham, V. (2008). The current status and future of clinical psychology: Towards a scientifically principled approach. Psychological Science in the Public Interest, 9, 67-103.

Beutler, L. E. (1991). Have all won and must all have prizes? Revisiting Luborsky et al.'s verdict. Journal of Consulting and Clinical Psychology, 59, 226-232.
Boberg, E. W., Gustafson, D. H., Hawkins, R. P., Peressini, T., Chan, C., \& Bricker, E. (1995). Development, acceptance and use patterns of a computer-based education and social support system for people living with AIDS/HIV infection. Computers in Human Behavior, 11, 289-312.

Bockting, C. L. H., Schene, A. H., Spinhoven, P., Koeter, M. W. J., Wouters, L. F., Huyser, J., \& Kamphuis, J. H. (2005). Preventing relapse/recurrence in recurrent depression with cognitive therapy: A randomized controlled trial. Journal of Consulting and Clinical Psychology, 73, 647-657.

Bordnick, P. S., Traylor, A., Copp, H. L., Graap, K. M., Carter, B., Ferrer, M., \& Walton, A. P. (2008). Assessing reactivity to virtual reality alcohol based cues. Addictive Behaviors, 33, 743-756.

Cannon, T. D., Cadenhead, K., Corhblatt, B., Woods, S. W., Addington, J., Walker, E., . . Heinssen, R. (2008). Prediction of psychosis in youth at high clinical risk: A multisite longitudinal study in North America. Archives of General Psychiatry, 65, 28-37.

Collins, L. A., Dziak, J. J., \& Li, R. (2009). Design of experiments with multiple independent variables: A resource management perspective on complete and reduced factorial designs. Psychological Methods, 14, 202-224.

Collins, L. A., Murphy, S. A., \& Srecher, V. (2005). The multiphase optimization strategy (MOST) and the sequential multiple assignment randomized trail (SMART): New methods for more potent e-Health interventions. American Journal of Preventive Medicine, 32, S112-S118

Cronbach, L. J. (1975). Beyond the two disciplines of scientific psychology. American Psychologist, 30, 116-126.

Cronbach, L. J., \& Snow, R. E. (1977). Aptitude and instructional methods: A handbook for research on interactions. New York, NY: Irvington.

Fournier, J. C., DeRubeis, R. J., Hollon, S. D., Dimidjian, S., Amsterdam, J. D., Shelton, R. C., \& Fawcett, J. (2011). Antidepressant drug effects and depression severity: A patient-level meta-analysis. Journal of the American Medical Association, 301, 47-53.

Hollon, S. D., DeRubeis, R. J., Shelton, R. C., Amsterdam, J. D., Salomon, R. M., O’Reardon, J. P., . . . Gallop, R. (2005). Prevention of relapse following cognitive therapy vs. medication in moderate to severe depression. Archives of General Psychiatry, 62, 417-422.

Insel, T. R., \& Cuthbert, B. N. (2009). Endophenotypes: Bridging genomic complexity and disorder heterogeneity. Biological Psychiatry, 66, 988-989.

Jarrett, R. B., Kraft, D., Doyle, J., Foster, B. M., Eaves, G. G., \& Silver, P. C. (2001). Preventing recurrent depression using cognitive therapy with and without a continuation phase. Archives of General Psychiatry, 58, 381-388.

Kazdin, A. E. (2007). Mediators and mechanisms of change in psychotherapy research. Annual Review of Clinical Psychology, 3, $1-27$.

Kazdin, A. E., \& Blase, S. L. (2011). Rebooting psychotherapy research and practice to reduce the burden of mental illness. Perspectives on Psychological Science, 6, 21-37.

Kessler, R. C., Berglunk, P., Borges, G., Nock, M., \& Wang, P. S. (2005). Trends in suicide ideation, plans, gestures, and attempts 
in the United States, 1990-1992 to 2001-2003. Journal of the American Medical Association, 293, 2487-2495.

Kessler, R. C., Demler, O., Frank, R. G., Olfson, M., Pincus, H. A., Walters, E. E., .. . Zaslavsky, A. M. (2005). Prevalence and treatment of mental disorders, 1990 to 2003. New England Journal of Medicine, 352, 2515-2523.

Kocsis, J. H., Leon, A. C., Markowitz, J. C., Manber, R., Arnow, B., Klein, D. N., \& Thease, M. E. (2009). Patient preference as a moderator of outcome for chronic forms of major depressive disorder treated with Nefazodone, cognitive behavioral analysis system of psychotherapy, or their combination. Journal of Clinical Psychiatry, 70, 354-361.

Kraemer, H. C., Wilson, G. T., Firburn, C. G., \& Agras, W. S. (2002). Mediators and moderators of treatment effects in randomized clinical trials. Archives of General Psychiatry, 59, 877-884.

McGurk, S. R., \& Mueser, K. (2008). Response to cognitive rehabilitation in older versus younger persons with severe mental illness. American Journal of Psychiatric Rehabilitation, 11, 90-105.

Miklowitz, D. J., Axelson, D. A., George, E. L., Taylor, D. O., Schneck, C. D., Sullivan, A., . . Birmaher, B. (2009). Expressed emotion moderates the effects of family-focused treatment for bipolar adolescents. Journal of the American Academy of Child and Adolescent Psychiatry, 48, 643-651.

Nemeroff, C. G., Heim, C. M., Thase, M. E., Klein, D. N., Rush, A. J., Schatzberg, A. F., . . Keller, M. B. (2003). Differential responses to psychotherapy versus pharmacotherapy in patients with chronic forms of major depression and childhood trauma. Proceedings of the National Academy of Sciences, USA, 100, 14293-14296.

Project Match Research Group. (1997). Matching alcoholism treatments to client heterogeneity: Project MATCH posttreatment drinking outcomes. Journal of Studies on Alcohol, 58, 7-29.

Sanislow, C. A., Pine, D. S., Quinn, K. J., Kozak, M. J., Garvey, M. A., Heinssen, R. K., . . Cuthbert, B. N. (2011). Developing constructs for psychopathology research: Research domain criteria. Journal of Abnormal Psychology, 119, 631-639.

Shoham, V., Rohrbaugh, M. J., Stickle, T. R., \& Jacob, T. (1998). Demand-withdraw couple interaction moderates retention in cognitive-behavioral vs. family-systems treatments for alcoholism. Journal of Family Psychology, 12, 557-577.

Shoham-Salomon, V., \& Hannah, M. T. (1991). Client-treatment interactions in the study of differential change processes. Journal of Consulting and Clinical Psychology, 59, 217-225.

Shulman, L. S., (1981, August). Educational psychology returns to school (G. Stanley Hall Series). Paper presented at the 89th Annual Convention of the American Psychological Association, Los Angeles, CA.

Strecher, V. J., Marcus, A., Bishop, K., Fleisher, L., Stengle, W., Levinson, A., . . Nowak, M. (2005). A randomized controlled trial of multiple tailored messages for smoking cessation among callers to the cancer information service. Journal of Health Communication, 10, 105-118.

Verghese, A. (2011, February 26). Treat the patient, not the CT scan. The New York Times. Retrieved from http://www.nytimes .com/2011/02/27/opinion/27verghese.html?pagewanted=all

Vittengl, J. R., Clark, L. A., \& Jarrett, R. B. (2010). Moderators of continuation phase cognitive therapy's effect on relapse, recurrence, remission, and recovery from depression. Behaviour Research and Therapy, 48, 449-458.

Wang, P. S., Berglund, P., Olfson, M., Pincus, H. A., Wells, K. B., \& Kessler, R. C. (2005). Failure and delay in initial treatment contact after first onset of mental disorders in the National Comorbidity Survey Replication. Archives of General Psychiatry, 62, 603-613.

Wykes, T., Reeder, C., Landau, S., Matthiasson, P., Haworth, E., \& Hutchinson, C. (2009). Does age matter? Effects of cognitive rehabilitation across the age span. Schizophrenia Research, 113, 252-258. 


\title{
Expanding the Toolkit or Changing the Paradigm: Are We Ready for a Public Health Approach to Mental Health?
}

Perspectives on Psychological Science 6(5) 483-487

(C) The Author(s) 2011

Reprints and permission:

sagepub.com/journalsPermissions.nav DOI: $10.1177 / 1745691611416996$

http://pps.sagepub.com

(S)SAGE

\author{
Marc S. Atkins and Stacy L. Frazier \\ University of Illinois at Chicago
}

\begin{abstract}
Kazdin and Blase aptly describe the enormous mental health burden facing our nation and suggest several ways to reform the workforce, setting, and content of services to address this long-standing unmet need. We propose that current health care reform legislation and associated advances in service delivery provide a unique and timely opportunity for a paradigm shift in mental health research, practice, and training to support services that are comprehensive, readily accessible, and relevant to a broad range of mental health needs and capacities. Using the recent public health initiative to contain the HINI virus for comparison, and informed by a long-standing and extensive literature documenting the need for a public health model for mental health, we describe the rationale for a three-tiered public mental health model, illustrated with examples from ongoing research, to minimize universal risk for mental health difficulties via capacity building in natural settings; reduce onset and severity of symptoms by prioritizing high-risk groups via screening and services for targeted populations; and reduce psychiatric impairment among individuals with more intensive needs via individual, family, and group interventions. New priorities for clinical science to support a public health approach are proposed.
\end{abstract}

\section{Keywords}

public health, policy, treatment

In March 2009, the World Health Organization (WHO) received reports of deaths in Mexico from a new strain of influenza virus, labeled H1N1 but known generically as swine flu. By June, the virus had spread to several countries, including the United States, which reported incidences in all states and territories. Public health officials braced for the worst. Facing the possibility of a worldwide epidemic, the WHO declared a pandemic alert and initiated a public health response with three interrelated goals: an intensive intervention to isolate and treat infected patients with antiviral drug therapy, a targeted intervention for at-risk populations (e.g., elderly, pregnant woman, young children) for immediate distribution of the newly developed vaccine, and a universal public health campaign to limit the spread of infection through proper hygiene (e.g., hand-washing, coughing into one's elbow). With these efforts, nearly 80 million people in 77 countries received the vaccine and a worldwide epidemic was averted. Although not without problems (World Health Organization, 2011), this was the largest and most successful public health response to an emerging crisis in over 40 years.

As we reviewed the Kazdin and Blase article, we reflected on lessons learned from the international public health community's coordination of a multilevel intervention to avert a worldwide epidemic. Notably, necessary reactive measures (e.g., isolate those already infected) were combined with voluntary proactive measures (e.g., promote proper hygiene) to limit the spread of infection. In contrast, consider the response (or lack thereof) of the mental health community to the enormous mental health burden facing our nation, aptly described by Kazdin and Blase and widely acknowledged for decades as highlighted in the historic Surgeon General's report of mental health published at the turn of the millennium. As Dr. Satcher notes in his preface, "Even more than other areas of health and medicine, the mental health field is plagued by disparities in the availability of and access to its services ... viewed readily through the lenses of racial and cultural diversity, age, . . . gender . . . (and) a person's financial status" (U.S. Public Health Service, 2000).

We commend Kazdin and Blase for raising the urgency of these issues among the clinical science community, but we are concerned that their recommendations, though often

\section{Corresponding Author:}

Marc S.Atkins, University of Illinois at Chicago, Institute for Juvenile Research (MC 747), I 747 W. Roosevelt Rd., Chicago, IL 60608

E-mail:atkins@uic.edu 
innovative, may serve to increase the toolkit rather than transform the paradigm. Similarly, although we greatly appreciate their call for the integration of prevention and intervention, we suggest, as others have before, that the problems are so long standing, so vast, and so unresponsive to current methods and models that a new comprehensive approach that utilizes levers of change at multiple levels is required. In particular, there will be no resolution of the nation's unmet mental health needs without recognition of the social determinants of health (Wilkinson \& Marmot, 2003) and the synergy that is created by distinct but complementary efforts along the continuum from prevention to intervention.

We also note that, in another way, the timing of the Kazdin and Blase article could not be more propitious given current innovations in health care and recently enacted health care reform legislation. As reviewed by Frank (2011), the Patient Protection and Affordable Care Act includes three primary levers of change: parity for mental health services as fully integrated with other health services, specific provisions for funding mental health promotion and prevention services, and the inclusion of community mental health centers in the definition of a "health home" (cf. Alakeson, Frank, \& Katz, 2010). In addition, the WHO recently issued a fact sheet on mental health that could become a driver of mental health policy, research, and practice and, we suggest, promote a reordering of priorities for clinical science (World Health Organization, 2010). Most notably, WHO emphasizes "intersectoral strategies" that deemphasize mental disorders to focus on "mainstreaming mental health promotion into policies and programmes in government and business sectors." Nationally and internationally, the balance is tipping in favor of a paradigm shift towards comprehensive models to alleviate mental health suffering,

The compelling case for a public health framework - and a three-tiered approach in particular - to address the persistent barriers to accessible and effective mental health services has been made before: in the Surgeon General's (2000) report, with further detail and emphasis in the recent Institute of Medicine's report on prevention of youth mental health disorders (Institute of Medicine, 2009), and most recently and succinctly by Stiffman, Stelk, Evans, and Atkins (2010). All of these reports recognize that a shift towards the efficient and effective implementation of a coordinated and comprehensive three-tiered approach to mental health will involve many challenges, including a reallocation of resources (e.g., Kelleher, 2010), a retooling of the workforce (Schoenwald, Ringeisen, Hoagwood, Evans, \& Atkins, 2010), and a broader reconceptualization of mental health promotion that includes healthy functioning across domains (e.g., cognitive, social, physical) and settings (e.g., home, school, work; M. Atkins, Hoagwood, Kutash, \& Seidman, 2010). Our goal in this commentary is not to reiterate these already well-articulated justifications for a public health approach to mental health. Instead, we hope to extend this vision by describing components of a three-tiered approach to mental health, including specific examples from our own work, as much to show the urgent need for additional research as to illustrate opportunities for change.

\section{Universal}

We have elsewhere proposed a model for mental health promotion at the universal level that enhances the natural synergy between community settings and mental health (Cappella, Frazier, Atkins, Schoenwald, \& Glisson, 2008; Frazier, Cappella, \& Atkins, 2007). Shifting prevention to natural settings (e.g., schools, park districts, community centers) makes sense for two primary reasons. First, mental health promotion already lies at the heart of most natural settings, whose goals, routines, and activities are inherently designed to foster skills building, positive relationships, and healthy functioning. Second, there is an extensive empirical literature to suggest that frontline staff often struggle to provide high-quality services or meet the extensive needs of youth or families in their care (e.g., Larson \& Walker, 2010; Pianta, Belsky, Houts, \& Morrison, 2007). Borrowing from an organizational perspective, we propose that supporting a natural setting means strengthening organizational capacity to achieve its mission and goals by supporting staff to effectively implement its core technology (i.e., deliver high-quality service) so that consumers of that service derive the most benefit out of their participation in that setting. By example, we have been pursuing a program of research in collaboration with the Chicago Park District that examines the capacity of recreational after-school and summer programs to promote children's mental health in urban, poor communities (Frazier et al., 2007).

To illustrate, extensive empirical data suggest that afterschool programs can play a critical role in children's psychosocial development, especially for children living in communities of concentrated urban poverty (Durlak, Mahoney, Bohnert, \& Parente, 2010). Despite their potential, however, program impact is often compromised by the extensive mental health needs of children and the pervasive poverty in which they live. Hence, we are pursuing two concurrent pathways. First, we are examining the feasibility and impact of community mental health agency consultation to recreation staff around academic enrichment, coaching behaviors, activity engagement, and behavior management (Frazier, Chacko, Van Gessel, Boyle, \& Pelham, in press). Second, we are working with lead administrators to examine and expand their organizational capacity to offer systematic training, professional development, and comprehensive support to their recreation leaders and physical instructors. Both efforts converge around the goal of improving service delivery and outcomes for youth participating in out-of-school-time programs.

\section{Targeted}

As is true for any public health problem, universal interventions are necessary but not sufficient to address the enormous 
mental health burden facing our nation. They will produce far less impact by themselves than if they are implemented as part of a comprehensive model, with unique but synergistic efforts at each level of intervention. When implemented successfully, universal interventions would reduce the risk for mental health problems and limit the numbers of individuals who enter this level of need. It follows, then, that targeted interventions would prioritize care for high-risk groups via indicated outreach, screenings, and services. Examples of high-risk groups might include children of parents with mental illness, families living in poverty, or individuals exhibiting subclinical symptoms or early evidence of impaired functioning. Targeted interventions can be integrated into both community and clinical settings, as illustrated in our earlier example. However, perhaps unlike natural settings that are designed for entire communities, targeted interventions may be more readily incorporated into settings such as primary-care offices, emergency rooms, and social service agencies inherently committed to identifying and reducing risky behaviors via health screenings, community outreach, psycho-educational activities, and early intervention.

The consultation of mental health providers to after-school staff noted above is one example of integrating universal and targeted interventions. As another example of a targeted intervention with universal components, we are studying a Medicaid fee-for-service, school-based mental health model for urban, low-income children and families that is guided by empirical evidence for schooling as critical for children's social and emotional adjustment and by evidence for the direct and indirect benefits of academic achievement for children's mental health (Cappella et al., 2008). In a series of iterative studies, we have identified teacher-referred children in early elementary grades exhibiting disruptive behaviors that impair classroom functioning and interfere with academic progress. Community mental health providers, parent advocates, and peer-identified teacher key opinion leaders (M. S. Atkins et al., 2008) together receive training and supervision in the implementation of evidence-based tools for the key empirical classroom and home predictors of children's learning. This ongoing work links universal (classroom-wide) and targeted (services for high need youth) levels to redefine mental health goals, mobilize natural and indigenous resources, and capitalize on the inherent capacity of natural settings to promote children's healthy development (M. Atkins et al., 2006, 2011).

\section{Intensive}

As noted by Kazdin and Blase, current rates of mental illness diagnoses in our country exceed the availability of mental health providers, resulting in an enormous mental health burden. The infusion of resources at universal and targeted levels of intervention is designed to reduce the prevalence of mental health disorders, thus reducing the number of individuals exhibiting clinical symptoms or more severe functional impairment. In turn, our nation's limited pool of mental health providers would be at liberty to serve the smaller subset of individuals whose intensive mental health needs warrant more extensive treatment. For example, returning to the after-school and school-based work noted earlier, we anticipate the need for more intensive services in classrooms and homes, as new findings indicate personal characteristics and settings that are unresponsive to the service model. Indeed, it is at this level of the pyramid - this end of the continuum from prevention to intervention - that Kazdin and Blase's innovative recommendations for new psychotherapy tools are most relevant and most ripe for close empirical examination.

It is also worth noting that trends would predict that the highest rates of unmet mental health need at this tier still would emerge from targeted groups at highest risk, thereby justifying the need to allocate resources for early intervention. Hence, to meet the needs at this most intensive level, we need to follow a variety of paths that extend beyond the most traditional clinical research and practice models, as Kazdin and Blase note quite clearly.

However, early efforts to move efficacious treatments from university-based clinical trials into community care settings revealed the extensive challenges associated with implementation. As highlighted with some frequency in the literature (e.g., Weiss, Doss, \& Hawley, 2005), the long-standing science-toservice gap in large part emanates from the fact that most evidence-based treatments have been developed with samples of patients and providers whose characteristics fail to represent those in routine care settings.

Fortunately, the last decade has given rise to several new areas of research, each helping to close the research to practice gap. For example, transportability studies emphasize training, supervision, fidelity, and feedback mechanisms to examine what it will take to achieve outcomes that approach those reported in efficacy studies (Schoenwald \& Hoagwood, 2001). Alternatively, Hoagwood and colleagues proposed the cliniccommunity intervention development model, which includes eight steps that begin with intervention development and end with dissemination and sustainability. Unique to this model is its emphasis on starting and ending in community settings with the providers and consumers for whom the interventions are intended (Hoagwood, Burns, \& Weisz, 2002). Most recently, Chorpita and colleagues introduced a "common elements" approach to service delivery, responding to the limited time and opportunity in community settings for clinician training and supervision in evidence-based interventions (Chorpita, Deleiden, \& Weisz, 2005). They identified 30 core intervention components that have high impact and broad relevance (e.g., differential attention, relaxation training social problem-solving), and packaged them in a website designed specifically for community-based service providers (Chorpita, Becker, \& Deleiden, 2007). This approach is currently being implemented nationally with ongoing evaluation and appears to have great promise to bring evidenced-based practice to scale. 


\section{Final Thoughts}

The long-standing mental health burden facing our nation is too vast and too impervious to change to be resolved by the fragmented approach that exists today. We thank Kazdin and Blase for highlighting the enormity and urgency of the problem for the clinical science community, though we suggest that a primary focus on revising psychotherapy, however innovative, addresses a relatively small proportion of the problem. As we have described, there is strong consensus that only a comprehensive and integrated public health model can adequately address the pervasive societal problems that underlie our country's mental health needs. Recent innovations in health care reform and newly enacted legislation provide a unique and timely opportunity to advance comprehensive models of mental health practice.

The ongoing programs of research we have presented are attempting to meet the need for new models of mental health service delivery. We offer them to augment the recommendations by Kazdin and Blase, to address the limitations of traditional psychotherapy, and to counter the tendency of our field to Balkanize prevention and intervention. Our field continues to allocate the most time and resources to the intensive tier of intervention (i.e., evidence-based treatments), whereas a public health approach suggests that we would have more success if comparable effort were allocated to coordinated care. We acknowledge that none of the models or examples is without limitation or immune from criticism and all are in need of further research and development. In fact, that is our very point in highlighting them for this commentary. The clinical science community has much to offer in clinical acumen and research expertise. In addition, interdisciplinary research with basic science, social science, and clinical allies will strengthen and speed the development of effective strategies to alleviate our nation's mental health burden. To that end, we urge the clinical science community to heed the long-standing call for a public health approach to mental health service delivery and, in particular, to prioritize a more equitable distribution of resources across the continuum from prevention to intervention.

\section{Declaration of Conflicting Interests}

The author declared no potential conflicts of interest with respect to the authorship or the publication of this article.

\section{References}

Alakeson, V., Frank, R., \& Katz, R. (2010). Specialty care medical homes for people with severe, persistent mental disorders. Health Affairs, 29, 867-873.

Atkins, M., Frazier, S., Birman, D., Adil, J. A., Jackson, M., Graczyk, P., . . McKay, M. (2006). School-based mental health services for children living in high poverty urban communities. Administration and Policy in Mental Health and Mental Health Services Research, 33, 146-159.

Atkins, M., Frazier, S., Schoenwald, S., Marinez-Lora, A., Shernoff, E., Cappella, E., . . G Gibbons, R. (2011). Mental health services and predictors of learning in urban schools (National Institute of Mental Health R01MH073749). Bethesda, MD: National Institute of Mental Health.

Atkins, M., Hoagwood, K., Kutash, K., \& Seidman, E. (2010). Towards the integration of education and mental health in schools. Administration and Policy in Mental Health and Mental Health Services Research, 37, 40-47.

Atkins, M. S., Frazier, S. L., Leathers, S. J., Graczyk, P. A., Talbott, E., Jakobsons, L., . . . Bell, C. C. (2008). Teacher key opinion leaders and mental health consultation in low-income urban schools. Journal of Consulting and Clinical Psychology, 75, 905-908.

Cappella, E., Frazier, S., Atkins, M., Schoenwald, S., \& Glisson, C. (2008). Enhancing schools' capacity to support children in poverty: An ecological model of school-based mental health services. Administration and Policy in Mental Health and Mental Health Services Research, 35, 395-409.

Chorpita, B., Becker, K., \& Deleiden, E. (2007). Understanding the common elements of evidence-based practice: Misconceptions and clinical examples. Journal of the American Academy of Child \& Adolescent Psychiatry, 46, 647-652.

Chorpita, B., Deleiden, E., \& Weisz, J. (2005). Identifying and selecting the common elements of evidence based interventions: A distillation and matching model. Mental Health Services Research, 7, 5-20.

Durlak, J. A., Mahoney, J. L., Bohnert, A. M., \& Parente, M. E. (2010). Developing and improving after school programs to enhance youth's personal growth and adjustment: A special issue of AJCP. American Journal of Community Psychology, 45, 285293.

Frank, R. (2011, April). Reforming the problem of disparities: Health care system change and improved behavioral health. Plenary presentation at From Disparities Research to Disparities Interventions: Lessons Learned and Opportunities for the Future of Behavioral Health Services conference. Arlington, VA.

Frazier, S. L., Cappella, E., \& Atkins, M. S. (2007). After school programs for children in urban poverty: Preventing problems and promoting opportunities. Administration and Policy in Mental Health and Mental Health Services Research, 34, 389-399.

Frazier, S. L., Chacko, A., Van Gessel, C., O’Boyle, C., \& Pelham, W. E. (in press). The summer treatment program meets the south side of Chicago: Bridging science and service in urban after-school programs. Child and Adolescent Mental Health.

Hoagwood, K., Burns, B.J., \& Weisz, J.R. (2002). A profitable conjunction: From science to service in children's mental health. In B.J. Burns \& K. Hoagwood (Eds.), Community treatment for youth: Evidence-based interventions for severe emotional and behavioral disorders (pp. 327-338). New York, NY: Oxford University Press.

Institute of Medicine. (2009). Preventing mental, emotional, and behavioral disorders among young people: Progress and possibilities. Washington, DC: Author.

Kelleher, K. (2010). Organizational capacity to deliver effective treatments for children and adolescents. Administration and Policy in Mental Health and Mental Health Services Research, 37, 89-94.

Larson, R. W., \& Walker, K. C. (2010). Dilemmas of practice: Challenges to program quality encountered by youth program leaders. American Journal of Community Psychology, 45, 338-349. 
Pianta, R. C., Belsky, J., Houts, R., \& Morrison, F. (2007). Opportunities to learn in America's elementary classrooms. Science, 315 , 1795-1796.

Schoenwald, S., \& Hoagwood, K. (2001). Effectiveness, transportability, and dissemination of interventions: What matters most? Psychiatric Services, 52, 1190-1197.

Schoenwald, S., Ringeisen, H., Hoagwood, K., Evans, M., \& Atkins, M. (2010). Workforce development and the organization of work: The science we need. Administration and Policy in Mental Health and Mental Health Services Research, 37, 71-80.

Stiffman, A., Stelk, W., Evans, M., \& Atkins, M. (2010). A public health approach to children's mental health services: Possible solutions to current service inadequacies. Administration and Policy in Mental Health and Mental Health Services Research, 37, 120-124.
U.S. Public Health Service. (2000). Mental health: A report of the Surgeon General. Washington, DC: Author.

Weiss, J. R., Doss, A. J., \& Hawley, K. M. (2005). Youth psychotherapy outcome research: A review and critique of the evidence base. Annual Review of Psychology, 56, 337-363.

Wilkinson, R., \& Marmot, M. (Eds.). (2003). The social determinants of health: The solid facts (2nd ed.). Copenhagen, Denmark: World Health Organization.

World Health Organization. (2010). Mental health: Strengthening our response (Fact Sheet No. 220). Geneva, Switzerland: Author.

World Health Organization. (2011). Report of the Fourth Meeting of the Review Committee on the Functioning of the International Health Regulations (2005) and Pandemic Influenza A. (H1N1) 2009. Geneva, Switzerland: Author. 


\title{
Use of Priming-Based Interventions to Facilitate Psychological Health: Commentary on Kazdin and Blase (20 I I)
}

Perspectives on Psychological Science 6(5) 488-492

(C) The Author(s) 2011

Reprints and permission:

sagepub.com/journalsPermissions.nav DOI: 10.1 |77/174569|6||4|6993

http://pps.sagepub.com

(SAGE

\author{
Idit Shalev and John A. Bargh
}

Yale University, New Haven, CT

\begin{abstract}
Whereas traditional psychological interventions have been conceptualized in terms of deliberate readiness for change (Prochaska \& DiClemente, 1983), emerging findings from social psychology suggest that regulation of behavior can operate independently of conscious selection and guidance (Bargh \& Morsella, 2010). This evidence has come from studies using priming techniques based on activation of relevant mental representations by external environmental stimuli (Bargh \& Chartrand, 2000). Research on automatic interpersonal processes has shown that feeling of social warmth (Bargh \& Shalev, 20I I; Williams \& Bargh, 2008a) and the regulation of maladaptive emotions (Williams, Bargh, Nocera, \& Gray, 2009), for example, can be induced nonconsciously by physical sensations, visual images or semantic concepts. Interventions based on the procedure of priming could be administered by multiple providers and communication devises to regulate emotional states, increase adherence to treatment instructions, or activate mind-sets that facilitate adaptive functioning. Integrating the methodology of priming and clinical intervention could both contribute to treatment delivery and enrich our understanding of change processes. We conclude that the use of supplementary priming-based interventions to facilitate and disseminate psychological change should be encouraged.
\end{abstract}

\section{Keywords}

priming, psychological intervention, embodiment, self regulation, implicit processes, automaticity, emotion regulation, change

Kazdin and Blase (2011) argue that a major shift and expansion of intervention research and clinical practice is needed to decrease the prevalence and incidence of mental illness. The goal of decreasing rates of mental illness and improving psychosocial functioning on a large scale (i.e., in society) begins with challenging basic assumptions as to which components underlie psychological intervention. We ask what school of thought could also be used to ameliorate psychological health problems. Much of our evidence has come from studies of automaticity using priming techniques, which refer to the passive, subtle, and unobtrusive activation of relevant mental representations by external environmental stimuli, including exposure to semantic concepts, short messages, visual images, and physical sensations (Bargh \& Chartrand, 2000). To integrate the notions of automaticity and clinical intervention, we discuss basic assumptions underlying priming-based intervention as compared with traditional interventions and demonstrate uses of priming-based procedures to activate and facilitate psychological change.

\section{Nonconscious Source of Psychological Change}

Traditional psychological interventions have been conceptualized in terms of the client's deliberate readiness for change
(Prochaska \& DiClemente, 1983). Because of this belief, systematic costly efforts are invested in individual treatment delivery, and individual-based interventions are not available for all those in need of services (Kazdin \& Blase, 2011). Despite little understanding of the mechanisms of change (i.e., precisely how they work; Kazdin, 2000, 2007), many traditional therapies (e.g., psychodynamic therapy) as well as evidence-based approaches (e.g., cognitive therapy) are focused on capturing nonconscious maladaptive patterns and challenging them through the use of strategies for arousal of awareness (e.g., emphasis on insight; Messer \& McWilliams, 2007; challenging automatic thoughts; Beck, 1997). Though most behavioral interventions highlight mechanisms other than awareness (e.g., exposure; Foa \& Kozak, 1986; behavioral activation; Lewinsohn, 1975; behavior modification; Kazdin, 1980), these techniques mostly involve conscious volitional engagement on the part of the patient.

In traditional personality psychology, techniques based on arousal of awareness were supported by the idea that a conscious,

Corresponding Author:

Idit Shalev, Institute of Social and Policy Studies, Yale University, New Haven, CT, 06520, USA

E-mail: idit.shalev@yale.edu or shalev.idit@gmail.com 
agentic self is posited to be the ultimate controller of individual human behavior (Bargh, Gollwitzer, \& Oettingen, 2010). Therefore, change will only be resulted by volitional engagement to defeat automatic responses. Similarly, traditional social psychology research on self-regulation suggests that success or positive outcomes only occur through the application of conscious control, with the blame for negative outcomes laid at the feet of automatic influences (e.g., Mischel \& Ayduk, 2004). For example, for individuals who are trying to lose weight, automatic impulses are seen as the cause for the overconsumption of fattening foods, whereas controlled, conscious processes are believed to be necessary to prevent these impulses from unduly affecting behavior (Baumeister, Heatherton, \& Tice, 1994).

Social psychologists have produced numerous demonstrations of nonconscious processes attaining the same outcomes as their conscious counterparts across a variety of research domains (Aarts \& Dijksterhuis, 2003; Bargh, Gollwitzer, LeeChai, Barndollar, \& Troetschel, 2001; Bargh \& Huang, 2009; Bargh \& Morsella, 2010; Dijksterhuis \& van Knippenberg, 1998), suggesting that both conscious and nonconscious processes play an important role in behavior change (Bargh et al., 2010). Whereas conscious processes are generally costly, intentional, controllable, and effortful, and the individual is aware of engaging in them, nonconscious automatic processes are characterized by their unintentional, relatively effortless (i.e., efficient; minimal attentional resources required) nature, and they operate outside of awareness (see Bargh, 1994; Bargh \& Williams, 2007). Use of subliminal primes has the advantage of assuring authenticity of the patients' responses, because strategic self-presentational modifications of responses are highly unlikely when the process occurs without awareness (Levy, 2009).

\section{What Mechanism Underlies Priming-Based Interventions?}

Priming-based interventions are based on the perception that relevant stimuli (primes) automatically activate a goal representation. The goal will then be pursued even though there is no conscious awareness of the primes, the active intention toward the goal, or the active guidance of goal-directed thought and behavior (Bargh \& Gollwitzer, 1994; Bargh \& Huang, 2009). The association between the external primes and the concept or the mental representation could be created naturally over development. For example, concepts concerning the physical world (e.g., physical distance, size, and physical temperature) form early in childhood as they are based on direct concrete experiences. These concepts do not require mental capacities of memory retrieval and comparison that do not develop until years later (Clark, 1973; Mandler, 1992). When these abstract concepts are later developed they tend to be "built upon" (and thus strongly associated with) these physical concepts to the extent they are analogous (i.e., share key features; Asch, 1946; Kelley, 1950; Lakoff \& Johnson, 1980; Williams \& Bargh, 2008a; Williams, Huang, \& Bargh, 2009) and hence could activate one another or be used strategically for psychological intervention.

One example for translating this associative mechanism into potential population-based intervention is the close association of physical and psychological warmth (and coldness). This association was first demonstrated by Harry Harlow (1958), who showed how physical warmth could be effectively substituted (in monkeys) for absent maternal warmth, leading to significantly greater social warmth capacities for the monkey later in adulthood. It was further supported by social neuroscience research implicating insular cortex in the processing of both physical temperature (e.g., Craig, Chen, Bandy, \& Reiman, 2000; Sung et al., 2007) and the psychosocial version of warmth information: feelings of trust (e.g., Sanfey, Rilling, Aronson, Nystrom, \& Cohen, 2003; Todorov, Baron, \& Oosterhof, 2008), empathy, and prosociality (Eisenberger, Lieberman, \& Williams, 2003; Kang, Williams, Clark, Gray, \& Bargh, 2010; Kross, Egner, Downey, Ochsner, \& Hirsch, 2007). Similarly, recent research on embodied cognition has shown these feelings of social warmth or coldness can be induced by experiences of physical warmth or coldness and vice versa (Bargh \& Shalev, 2011; IJzerman \& Semin, 2009; Williams \& Bargh, 2008a; Zhong \& Leonardelli, 2008).

In a recent set of studies, we tested the use of this association for emotion regulation intervention. Remarkably, we have found that people already implicitly use this automatic association between physical and social warmth to regulate their emotional states through the frequency, duration, and preferred water temperature of the showers and baths that they take. Applications of physical warmth temporarily reduced or even eliminated feelings of loneliness and exclusion without the individual's explicit awareness of the physical-psychological relation. Furthermore, socially excluded participants who were primed with physical warmth showed a significant decrease in their need for affiliation and a desire for emotionimproving activities in comparison with a group of excluded participants who were primed with physical coldness or a control group (Bargh \& Shalev, 2011).

This example demonstrates that primed experiences of physical warmth could be a boon to a population-based intervention of syndromes associated with emotion regulation (e.g., borderline personality disorder; see Glenn \& Klonsky, 2009; Linehan, 1993). Physical warmth primes can facilitate bonding and interpersonal trust in the health provider that is the bread and butter of every psychological intervention (Bowlby, 1969, 1988; Gelso, 2011; Gelso \& Samstag, 2008; Orlinsky, Ronnestad, \& Willutski, 2004). This therapeutic bonding establishes a secure base from which the therapist influences the client through various psychological interventions (e.g., suggestion, encouragement of open communication, modeling, reward manipulation, exposure and cognitive restructuring). Moreover, these findings also demonstrate that change can be produced without conscious awareness on the part of the patient and by simple techniques other than the traditional individual model for treatment delivery. 


\section{Which Psychological Problems Could be Targeted by Primes?}

Kazdin and Blase (2011) argue that the challenge for psychological interventions is to help reduce the burden of mental illness and related conditions both at the personal and societal level. By promoting adaptive behaviors and mental-healthrelated responses such as easing stress or irritability, feeling better about oneself, feeling secure, feeling motivated, feeling affiliated and the like, positive primes may push some significant number of individuals into a slightly more positive and less stressed realm and for many that would have impact on determining whether symptoms or disorders were associated with impairment.

Following this view, studies based on the nonconscious, automatic perspective have demonstrated not only that maladaptive behavioral outcomes (e.g., overeating) can be driven by incidental exposure to contextual cues (i.e., priming) associated with that behavior (televised food ads; Harris, Bargh, \& Brownell, 2009). Primes can also promote prosocial/mental health related responses and activate aids to self-regulation such as reappraisal processes (Mauss, Cook, Cheng, \& Gross, 2007; Williams, Bargh, et al., 2009) or emotional distance (Williams \& Bargh, 2008b). For example, in one set of experimental studies, nonconscious reappraisal priming was found to be significantly more effective than people's spontaneous regulatory efforts, with the nonconscious emotion regulation condition demonstrating less reactivity than the conscious reappraisal group (Williams, Bargh, et al., 2009). In another set of studies, priming of physical distance by merely having the participant plot an assigned set of points on a Cartesian coordinate plane activated representations of physical distance and influenced feelings of emotional and interpersonal distance (Williams \& Bargh, 2008b). In the most recent set of studies, temperature primes reduced perceived loneliness and sense of social exclusion (Bargh \& Shalev, 2011).

It is possible that a variety of conditions associated with emotion regulation and interpersonal relations (e.g., selfcontrol, impulsivity, or interpersonal violence) could be ameliorated by physical temperature interventions. Physical primes, especially those revolving around issues of trust and empathy, may also be of great value to the treatment of young children's attachment and other emotional problems, because as the nonconscious emotion regulation research shows, nonconscious interventions are of particular value to those who are unable to regulate through the traditional, conscious means.

Primes can also promote adaptive functioning of specific groups (e.g., preverbal children, elderly). For example, priming was used in one set of studies for improvement of adaptive functioning among the elderly. When old and young participants were first primed with either positive or negative elderly stereotypic words, memory performance in the older (but not younger) participants was improved by the positive stereotypic associations and was hindered by the negative stereotypic associations. These effects resulted from flashing age-stereotype words, such as learned and confused, on a computer screen at subliminal speeds-fast enough to prevent conscious perception, but slow enough to allow encoding (Levy, 1996). A variety of social phenomena (e.g., stereotypes, stigmatized mental health groups, attitudes toward minorities) could be activated by environmental contexts (i.e., primes) of physical impairment. For example, Stapel and Lindenberg (2011) recently found that disordered contexts (such as litter or a broken-up sidewalk, abandoned bicycle and a dirty train) promoted stereotyping and discrimination toward minorities. Future research will examine if these conditions could be challenged by physical cues of cleanliness or environmental organization.

Priming-based interventions could enrich existing procedures for different conditions. A wide array of maladaptive habitual behaviors (e.g., smoking, procrastination) could be targeted by primes that activate avoidance or aversion reactions. Similarly, treatment of anxiety and mood difficulties, eating disorders, addictive behaviors, or learning disabilities are all affected by environmental influences. Use of healthrelated primes could reduce emotion contagion within depressed couples or family accommodation to symptoms by consistent reminders of cues associated with functional behavior patterns.

Individual differences also need to be examined to address differences in response to environmental conditions (Aarts, Wegner, \& Dijksterhuis, 2006). These studies could examine the interrelations between specific deficits (e.g., attentional bias) and response to contextual cues. For example, theories of depression (Abramson, Seligman, \& Teasdale, 1978; Beck, 1967) suggest that differences between dysphoric and nondysphoric patients result from cognitive schemata that are triggered by self-referential processes (e.g., Bargh \& Tota, 1988). Consistent with this assumption, dysphoric compared with nondysphoric patients were found to be lower in sense of authorship (the feeling that observed effects are caused by one's own actions) after priming of the self concept in an ambiguous situation. However, priming the potential effects of an action just prior to their occurrence increased the sense of authorship in all participants (Aarts et al., 2006). This study demonstrates the differences between normal and clinical populations in response to the prime, indicating that people who are unable to (implicitly) self-regulate their behavior through the prime may be candidates for additional effective interventions. Priming-based intervention combined with other techniques may facilitate the treatment effects for clinical population.

\section{How to Incorporate Priming-Based Interventions Into Daily Life?}

Experimental studies have demonstrated the utility of priming-based interventions in laboratory setting and field experiments (Bargh \& Shalev, 2011; Levy, 2009). The findings demonstrate that people implicitly use natural sources to selfregulate their emotional states without conscious awareness of 
doing so. The remaining challenge is to achieve the activation of contextual cues in broader, everyday settings and on a sustained basis. To address this goal, natural environmental conditions (e.g., temperature, space) as well as various technologies could be used to improve an individual's functioning. Communication devices (e.g., smartphones) could be used to increase the accessibility and distribution of treatment messages (e.g., by text messaging, visual images). Similarly, computers screen savers, home pages, photographs in the office, exposure to different real or virtual environments ("wide open spaces" vs. crowded urban environments) could easily be developed and/or used to activate the desired mental representation.

Clearly, the use of mundane physical experiences, easily available to all, as therapeutic interventions would increase the availability of therapy to all who need it (Kazdin \& Blase, 2011). Priming-based supplementary interventions could be administered by multiple providers (e.g., parents, educators, nurses, media and communication devices). Such interventions have the advantages of low cost and effort, individualization, anonymity, and widespread reach to facilitate action, increase adherence to treatment instructions, or activate mindsets that facilitate adaptive functioning. Future research will be needed to examine the ecological validity of priming-based interventions and their implications for clinical population and prevention. Multiple strategies (e.g., priming procedures, coaching and health education, media and communication devices) regulated by professional case managers can be extended in a programmatic way to move from intensive, costly, and individual case application to versions that are more population based. Integrating the methodology of priming and clinical interventions could contribute to the effective delivery of treatment to a much wider patient base than is presently the case.

\section{Declaration of Conflicting Interests}

The authors declared that they had no conflicts of interest with respect to their authorship or the publication of this article.

\section{References}

Aarts, H., \& Dijksterhuis, A. (2003). The silence of the library: Environment, situational norm, and social behavior. Journal of Personality and Social Psychology, 84, 18-28.

Aarts, H., Wegner, D. M., \& Dijksterhuis, A. (2006). On the feeling of doing: Dysphoria and the implicit modulation of authorship ascription. Behaviour Research and Therapy, 44, 1621-1627.

Abramson, L. Y., Seligman, M. E. P., \& Teasdale, J. D. (1978). Learned helplessness in humans: Critique and reformulation. Journal of Abnormal Psychology, 87, 49-47.

Asch, S. E. (1946). Forming impressions of personality. Journal of Abnormal and Social Psychology, 41, 258-290.

Bargh, J. A. (1994). The four horsemen of automaticity: Awareness, intention, efficiency, and control in social cognition. In R. S. Wyer Jr. \& T. K. Srull (Eds.), Handbook of social cognition (2nd ed., pp. 1-40). Hillsdale, NJ: Erlbaum.
Bargh, J. A., \& Chartrand, T. L. (2000). The mind in the middle: A practical guide to priming and automaticity research. In H. T. Reis \& C. M. Judd (Eds.), Handbook of research methods in social and personality psychology (pp. 253-285). New York, NY: Cambridge University Press.

Bargh, J. A., \& Gollwitzer, P. M. (1994). Environmental control of goal directed action: Automatic and strategic contingencies between situations and behavior. In W.D. Spaulding (Ed.), Integrative views of motivation, cognition, and emotion (pp. 71-124). Lincoln: University of Nebraska Press.

Bargh, J. A., Gollwitzer, P. M., Lee-Chai, A., Barndollar, K., \& Troetschel, R. (2001). The automated will: Nonconscious activation and pursuit of behavioral goals. Journal of Personality and Social Psychology, 81, 1014-1027.

Bargh, J. A., Gollwitzer, P. M., \& Oettingen, G. (2010). Motivation. In S.T. Fiske, D.T. Gilbert, \& G. Lindzey (Eds.), Handbook of social psychology (5th ed., pp. 268-316). New York, NY: Wiley.

Bargh, J. A., \& Huang, J. Y. (2009). The selfish goal. In G. B. Moskowitz \& H. Grant (Eds.), The psychology of goals (pp. 127150). New York, NY: Guilford.

Bargh, J. A., \& Morsella, E. (2010). Unconscious behavioral guidance systems. In C. Agnew, D. Carlston, W. Graziano, \& J. Kelly (Eds.), Then a miracle occurs: Focusing on behavior in social psychological theory and research (pp. 89-118). New York, NY: Oxford University Press.

Bargh, J. A., \& Shalev, I. (2011). The substitutability of physical and social warmth in daily life. Emotion. Advanced online publication. doi:10.1037/a0023527

Bargh, J. A., \& Tota, M. E. (1988). Context-dependent automatic processing in depression: Accessibility of negative constructs with regard to self but not others. Journal of Personality and Social Psychology, 54, 925-993.

Bargh, J. A., \& Williams, L. E. (2007). The nonconscious regulation of emotion. In J. Gross (Ed.), Handbook of emotion regulation (pp. 429-445). New York, NY: Guilford Press.

Baumeister, R. F., Heatherton, T. F., \& Tice, D. M. (1994). Losing control: How and why people fail at self-regulation. San Diego, CA: Academic Press.

Beck, A. T. (1967). Depression: Clinical, experimental, and theoretical aspects. New York, NY: Hoeber.

Beck, A. T. (1997). The past and future of cognitive therapy. Journal of Psychotherapy Practice and Research, 6, 276-284.

Bowlby, J. (1969). Attachment and loss. London, England: Hogarth Press.

Bowlby, J. (1988). A secure base: Clinical applications of attachment theory. London, England: Routledge.

Clark, H. H. (1996). Using language. Cambridge, England: Cambridge University Press.

Craig, A. D., Chen, K., Bandy, D., \& Reiman, E. (2000). Thermosensory activation of insular cortex. Nature Neuroscience, 3, 184-190.

Dijksterhuis, A., \& van Knippenberg, A. (1998). The relation between perception and behavior, or how to win a game of trivial pursuit. Journal of Personality and Social Psychology, 74, 865-877.

Eisenberger, N. I., Lieberman, M. D., \& Williams, K. D. (2003). Does rejection hurt? An fMRI study of social exclusion. Science, 203, 290-292. 
Foa, E. B., \& Kozak, M. J. (1986). Emotional processing of fear: Exposure to corrective information. Psychological Bulletin, 99, 20-35.

Gelso, C. J. (2011). The real relationship in psychotherapy: The hidden foundation of change. Washington, DC: American Psychological Association.

Gelso, C. J., \& Samstag, L. W. (2008). A tripartite model of the therapeutic relationship. In S. Brown \& R. Lent (Eds.), Handbook of counseling psychology (pp. 267-280). New York, NY: Wiley.

Glenn, C. R., \& Klonsky, E. D. (2009). Emotion dysregulation as a core feature of Borderline Personality Disorder. Journal of Personality Disorder, 23, 20-28.

Harlow, H. (1958). The nature of love. American Psychologist, 13, 673-685.

Harris, J. L., Bargh, J. A., \& Brownell, K. D. (2009). Priming effects of television food advertising on eating behavior. Health Psychology, 28, 404-413.

IJzerman, H., \& Semin, G. R. (2009). The thermometer of social relations: Mapping social proximity on temperature. Psychological Science, 20, 1214-1220.

Kang, Y., Williams, L., Clark, M., Gray, J., \& Bargh, J. (2010). Physical temperature effects on trust behavior: The role of insula. Social Cognitive and Affective Neuroscience, 5, 1-9. doi:10.1093/scan/ nsq077v2.

Kazdin, A. E. (1980). Behavior modification in applied settings (2nd ed.). Homewood, IL: Dorsey.

Kazdin, A. E. (2000). Psychotherapy for children and adolescents: Directions for research and practice. New York, NY: Oxford University Press.

Kazdin, A. E. (2007). Mediators and mechanisms of change in psychotherapy research. Annual Review of Clinical Psychology, 3, $1-27$.

Kazdin, A. E., \& Blase, S. (2011). Rebooting psychotherapy research and practice to reduce the burden of mental illness. Perspectives on Psychological Science, 6, 21-37.

Kelley, H. H. (1950). The warm-cold variable in first impressions of persons. Journal of Personality, 18, 431-438.

Kross, E., Egner, T., Downey, G., Ochsner, K., \& Hirsch, J. (2007). Neural dynamics of rejection sensitivity. Journal of Cognitive Neuroscience, 19, 945-956.

Lakoff, G., \& Johnson, M. (1980). Metaphors we live by. Chicago, IL: University of Chicago Press.

Levy, B. R. (1996). Improving memory in old age by implicit selfstereotyping. Journal of Personality and Social Psychology, 71, 1092-1107.

Levy, B. R. (2009). Stereotype embodiment: A psychosocial approach to aging. Current Directions in Psychological Science, 18, 332336.

Lewinsohn, P. M. (1975). The behavioral study and treatment of depression. In M. Hersen, R. M. Eisler, \& P. M. Miller (Eds.), Progress in behavioral modification (Vol. 1, pp. 19-65). New York, NY: Academic Press.
Linehan, M. M. (1993). Cognitive behavioral treatment of borderline personality disorder. New York, NY: Guilford.

Mandler, J. M. (1992). How to build a baby: II. Conceptual primitives. Psychological Review, 99, 587-604.

Mauss, I. B., Cook, C. L., Cheng, J. Y. J., \& Gross, J. J. (2007). Individual differences in cognitive reappraisal: Experiential and physiological responses to an anger provocation. International Journal of Physiology, 66, 116-124.

Messer, S., \& McWilliams, N. (2007). Insight in psychodynamic therapy: Theory and assessment. In L. G. Castonguay \& C. E. Hill (Eds.), Insight in psychotherapy (pp. 9-29). Washington, DC: American Psychological Association.

Mischel, W., \& Ayduk, O. (2004). Willpower in a cognitive-affective processing system: The dynamics of delay of gratification. In R. F. Baumeister \& K. D. Vohs (Eds.), Handbook of self-regulation: Research, theory, and applications (pp. 99-129). New York, NY: Guilford Press.

Orlinsky, D. E., Ronnestad, M. H., \& Willutski, U. (2004). Fifty years of psychotherapy process-outcome research: Continuity and change. In M. J. Lambert (Ed.), Handbook of psychotherapy and behaviour change (5th ed., pp. 307-389). New York, NY: Wiley.

Prochaska, J. O., \& DiClemente, C. C. (1983). Stages and processes of self-change of smoking: Toward an integrative model of change. Journal of Consulting and Clinical Psychology, 51, 390-395.

Sanfey, A. G., Rilling, J. K., Aronson, J. A., Nystrom, L. E., \& Cohen, J. D. (2003). The neural basis of economic decision-making in the ultimatum game. Science, 300, 1755-1758.

Stapel, D. A., \& Lindenberg, S. (2011). Coping with chaos: How disordered contexts promote stereotyping and discrimination. Science, 332, 251-253.

Sung, E., Yoo, S., Yoon, H. W., Oh, S., Han, Y., \& Park, H. W. (2007). Brain activation related to affective dimension during thermal stimulation in humans: A functional magnetic resonance imaging study. International Journal of Neuroscience, 117, 1011-1027.

Todorov, A., Baron, S. G., \& Oosterhof, N. N. (2008). Evaluating face trustworthiness: A model based approach. Social Cognitive and Affective Neuroscience, 3, 119-127.

Williams, L. E., \& Bargh, J. A. (2008a). Experiencing physical warmth promotes interpersonal warmth. Science, 322, 606-607.

Williams, L. E., \& Bargh, J. A. (2008b). Keeping one's distance: The influence of spatial distance cues on affect and evaluation. Psychological Science, 19, 302-308.

Williams, L. E., Bargh, J. A., Nocera, C. C., \& Gray, J. R. (2009). The unconscious regulation of emotion: Nonconscious reappraisal goals modulate emotional reactivity. Emotion, 9, 847-854.

Williams, L. E., Huang, J. Y., \& Bargh, J. A. (2009). The scaffolded mind: Higher mental processes are grounded in early experience of the physical world. European Journal of Social Psychology, $39,1257-1267$.

Zhong, C.-B., \& Leonardelli, G. J. (2008). Cold and lonely. Does social exclusion literally feel cold? Psychological Science, 19, 838-842. 


\title{
The Old Solutions Are the New Problem: How Do We Better Use What We Already Know About Reducing the Burden of Mental Illness?
}

Perspectives on Psychological Science 6(5) 493-497

(C) The Author(s) 2011

Reprints and permission:

sagepub.com/journalsPermissions.nav DOI: |0.1|77/|74569|6||4|8240

http://pps.sagepub.com

\author{
Bruce F. Chorpita', Mary Jane Rotheram-Borus', \\ Eric L. Daleiden ${ }^{2}$, Adam Bernstein', Taya Cromley', \\ Dallas Swendeman', and Jennifer Regan' \\ 'Department of Psychology, University of California, Los Angeles, and ${ }^{2}$ PracticeWise, LLC, Wilsonville, OR
}

\begin{abstract}
Kazdin and Blase (20II) propose that traditional models of delivering therapy require more resources than are available to address the scope of mental illness. We argue that finding new platforms and avenues for our existing treatments is a good start but that it is not enough. We contend that the field also needs to develop formal strategies to reorganize its increasing abundance of knowledge to address the scarcity of resources for its application. If we can better utilize our existing knowledge, treatment delivery and service resource allocation can become more efficient and effective. If the field continues with its almost singular emphasis on knowledge proliferation (e.g., developing new treatments), as opposed to knowledge management (e.g., developing new ways to design, apply, and organize existing treatments), the problem outlined by Kazdin and Blase cannot be solved.
\end{abstract}

\section{Keywords}

methodology, treatment

Kazdin and Blase (2011) assert that unless we make some major changes, our profession cannot meet the demand for mental health services in the U.S. or globally. They offer the idea of a portfolio of models, and we agree entirely that increasing the range of how existing treatments can be applied will help reduce the overall burden of mental health suffering. However, within the current zeitgeist, this could well mean that we will see 10 different versions of each protocol, each requiring 10 efficacy trials and 10 more effectiveness trialsessentially taking us from thousands of treatments to hundreds of thousands. This is certainly not what Kazdin and Blase intend, but we believe that without deliberate intervention, it is likely to be how the field responds.

\section{We Need More and Better Ways to Organize and Move Knowledge}

We see this as a knowledge management problem. That is, continued proliferation of knowledge about treatment will not help unless we get much, much better at summarizing, synthesizing, integrating, and delivering what we already have (Graham et al., 2006). The existing knowledge base is now too large to comprehend and apply optimally by any psychologist. In our recent efforts to examine how to choose a set of evidence-based treatments (EBTs) that best fit an organization's service population (Chorpita, Bernstein, \& Daleiden, in press), we discovered that simply selecting a set of no more than a dozen treatments from among all EBTs for children yields over 67 sextillion possibilities. To put this number in some perspective, if one were to write each unique set of 12 or fewer treatments on a single sheet of ordinary paper, the resulting pile would reach to the sun and back. Over 20 million times. Each of these sets has a unique composition and thus a potentially unique impact on the service population. Selecting an ideal array of treatments from among the promising possibilities is no longer a simple problem and it is approaching unsolvability. Although we know much about what works, we can no longer apply that knowledge efficiently.

Corresponding Author:

Bruce F. Chorpita, Department of Psychology, University of California, Los Angeles, Box 951 563, Los Angeles, CA 90095

E-mail:chorpita@ucla.edu 
Any system seeking to provide quality care informed by research must select treatments to put into its service array. Given that providing evidence-based, quality care to those in need has become a public health priority (U.S. Department of Health and Human Services, 2007) and that there are numerous ongoing efforts to implement EBTs at the state and national level (Chambers, Ringeisen, \& Hickman, 2005), the number of systems facing this challenge is only increasing. It is time to consider whether our policies to implement EBTs and our rules for how we define them are really compatible.

\section{We Need Options Other Than Treatment Manuals to Transfer Knowledge}

Over the past 20 years, the field has emphasized efficacy and internal validity over external validity, feasibility, and acceptability in order to identify what works. Following the principles of good research design (Kazdin, 2003), the field required primarily that treatments be well-specified, typically in the form of a book or manual (e.g., Chambless \& Hollon, 1998). This development was an extraordinary and innovative leap forward. But like nearly all innovations, it has had unforeseen consequences as well, which we must now face squarely.

Our knowledge has been packaged in units that cannot easily be combined. Is each manual, tested within its own research program, really a world unto itself? If so, then we have hundreds, perhaps thousands, of silos of expert knowledge, with little means to organize or combine them. Despite many years of brilliant innovation in treatment development and research, a child with two different problem clusters (e.g., separation anxiety and depressed mood) will at best receive a sequence of two separate EBTs built by two different experts. In this day and age, there is still no way for a child to receive care in the community that formally combines the collective scientific expertise on what to do for both conditions, even though we now have very good ideas for how to treat each. Although transdiagnostic treatment models are at last emerging (e.g., Allen, McHugh, \& Barlow, 2008), for the most part, the products of our research are still getting in the way of utilizing the knowledge behind them.

In our own work, we have sought to aggregate knowledge in the form of practice elements (discrete clinical procedures), noting which ones are commonly associated with successful outcomes for which symptoms in clinical trials (e.g., distillation and matching model; Chorpita \& Daleiden, 2009). This work has involved coding all available treatments protocols for their common procedures and operations (e.g., use of a reward program, relaxation training, cognitive restructuring) and identifying how those operations are associated with client or context features (e.g., diagnosis, age, setting). This process produces profiles or frequency distributions showing which procedures are most commonly associated with successful treatments for which clinical presentations.

Similar efforts to aggregate practice elements across independent treatments are underway in social work (Barth et al., in press) and in our multidisciplinary collaboration with the National Child Traumatic Stress Network (e.g., Layne et al., in press). The point of this work is that the patterns in the whole may reveal more than just the sum of the parts-for example, the supportive evidence for a single treatment protocol may be bolstered by the findings from procedurally related treatments. We can imagine possibilities where the actions of therapists are guided not always by a single manual but at times by the entire relevant treatment literature.

By aggregating across treatments to look for common elements, we seek to outline the robust or important features of an EBT and to distinguish those features that are nonessential "nuance." What features are important for efficacy is ultimately an experimental question, and until we have that knowledge in place and can then deliver it in real time to inform treatment prescription, data aggregation methods such as the distillation and matching model are the intermediate step. Kazdin (e.g., 2008a, 2008b) has repeatedly cautioned that an ontology of change mechanisms will take more than our lifetimes to establish. Waiting to discern what we do not know should not stop us from reconsidering what we already do.

Many strategies can be used to aggregate knowledge; identifying practice elements is only one of them. Identifying common processes is another (Collins, Phields, Duncan, \& Science Application Team, 2007; Ingram, Flannery, Elkavich, \& Rotheram-Borus, 2008; Rotheram-Borus, Ingram, Swendeman, \& Flannery, 2009). Processes refer to such things as the degree of structure, activities directed at setting a tone for the group, or the role of the facilitator as active or not. Another approach in both clinical and health promotion trials is to identify standardized functions (Hawe, Shiell, \& Riley, 2004), such as providing education, improving detection, building social networks and support, or facilitating accumulation of instrumental goods. In other words, treatments can be organized more around aims than strategies to achieve those aims. Complex interventions may have limited impact because we too literally advocate for replication with fidelity of activities and scripts. There may be multiple strategies to achieve health knowledge, all of which are acceptable, especially in allowing cultural tailoring, if the function of increasing health knowledge is served. Thus, in our existing compendia of EBTs, almost any dimension can be aggregated and mined: how treatments are arranged, the style with which they are delivered, the manner in which they are supervised, or the functions they serve. Each analysis reveals patterns that summarize features of the best treatments.

So what will these patterns tell us? At one end of the spectrum, they can point to intact EBTs. For example, the treatment that shares the most features in common with all of the 45 EBTs relevant to a 12-year-old girl with anxiety could be a reasonable choice, because it is not only evidence-based within its own replication series but it is also backed by over 30 neighboring randomized trials of highly similar treatments. The same cannot be said for an anxiety treatment whose 
features suggest it is more of an outlier within that group - it may have to stand alone on its own clinical trials.

Further along the continuum, aggregate patterns can tell us how to build new, perhaps those that are more flexible and more broadly applicable, that borrow the most commonly used procedures of treatments for various disorders. We have developed and recently tested one such protocol that targets four disorders (anxiety, depression, conduct problems, and traumatic stress) using a combination of the components drawn from existing evidence-based approaches and coordinated by a set of guiding algorithms (MATCH-ADTC; Chorpita \& Weisz, 2009). We do not really see this as a new treatment-it is better characterized as a new arrangement of the old treatments - an attempt to do more with what we already have.

At the furthest end of the spectrum of independent versus flexibly aggregated treatments, we see the possibility for realtime design of treatments based solely on libraries of component procedures and libraries of the algorithms for combining and ordering those procedures. We have recently designed and implemented such a direct service prototype in children's mental health, focusing on the selection and delivery of practice elements using guiding algorithms and in the context of feedback on progress and practice history (Chorpita \& Daleiden, 2010). Ultimately, we see possibilities for multideveloper treatment content libraries that can be delivered flexibly across multiple media and service platforms.

\section{We Need to Codesign EBTs}

To meaningfully achieve the goal that Kazdin and Blase outline - a portfolio of models - we may also need to move away from a paradigm in which laboratory experts solely design treatments. Treatment may ultimately involve codesign: important initial parameters and procedures built in the lab and real-time adjustments and local adaptation made in the field by clinicians. This will yield treatments that involve shared expertise-leveraging two knowledge bases. A priori, the investigator contributes the essentials as to what aspects of treatment should be included or how certain procedures should be performed at the time of service delivery. In real time, the clinician then adds the local expertise to adapt process, content, or logic based on the thousands of context variables that the laboratory developer cannot anticipate. Many EBTs now overspecify procedural details - sometimes right down to what games to play or which characters to use to illustrate a point (cf. Schoenwald, Garland, Southam-Gerow, Chorpita, \& Chapman, 2011). Identifying the nonessential details in EBTs will move those treatments closer to Kazdin and Blase's concept of a portfolio of models.

So how do we know which details are nonessential? It very well may be those that do not show up in most treatments when we aggregate across all of them relevant to a particular set of client characteristics-yet another reason to pursue knowledge aggregation. By stripping some of our best treatments down to the essence, we can allow them to be fleshed out again at the point of service by practitioners with local expertise who are embedded in the local context. Let therapists add their own jokes, games, or metaphors, and let researchers outline the core change strategies that should be preserved within those operations. If we do not know the core strategies, let knowledge aggregation point to promising candidates to be tested in focal or dismantling research designs.

Having researchers and clinicians codesign treatments in this way is consistent with the recently stated ideals of the American Psychological Association in the statements regarding evidencebased practices (APA Presidential Task Force, 2006). However, despite these ideals, the landscape of clinical practice still appears to be mostly characterized by a false dichotomy of evidence-based practice or clinical judgment. We need more formal models and exemplars for evidence-based practice and clinical judgment together. Of course, we do not know how much treatment design should occur a priori in the lab versus in real time in the field. Thus, we also need a new research agenda to study which codesign proportions work best (although we already have some idea that a heavy proportion of investigatorspecified design does not; e.g., Addis \& Krasnow, 2000; Borntrager, Chorpita, Higa, \& Weisz, 2009).

\section{Treatments Will Have to Work Together}

We have many treatments that work but only a limited understanding of how they work together. Kazdin and Blase describe the image of a pie, with slices representing people covered by different treatments. Whether treatment is ultimately delivered as therapist-selected practice elements, discrete manualized programs, or in some combination, we have not put enough thought into how to assemble arrays of treatments within service systems or how to gauge their collective impact on a community.

How many EBTs are required to serve a given population? The real answer depends on the local epidemiology; however, our analyses suggests that even learning all of them would generally not be enough to ensure that everyone with mental health needs receives evidence-based care - far from it (Chorpita et al., in press). Simply making new treatments is not likely to solve this problem and only exacerbates the problem of selecting the best set. To that end, we have developed an analytic method for simultaneously combining local population data and treatment outcome data to point to best-fitting solutions (Chorpita et al., in press). This methodology applies mathematical modeling to enhance resource allocation and help a service system achieve the greatest reduction in the burden of mental illness, much as Kazdin and Blase suggested. This tool can be considered a knowledge management appliance, and we need more like it to address our new problems.

\section{Knowledge Must Flow in Many Directions}

Despite all our treatment outcome research, the best source of evidence is still arguably the evidence that a client is 
improving. That, too, is a source of knowledge that is largely untapped. Despite emerging research that measurement feedback systems can improve outcomes (Bickman, 2008; Lambert, Harmon, Slade, Whipple, \& Hawkins, 2005), there is no widely used appliance for providing clinicians feedback on their clients' mental health outcomes - or clients with feedback on their own outcomes. This is a major research agenda requiring intensive innovation - there is currently only a handful of prototypes of this kind of technology, which is in stark contrast with the hundreds of manualized treatments available.

Such feedback should also not be limited to outcomes. As the compendia of EBTs for mental health have grown, so has the literature on the failure to implement EBT with fidelity. For example, only half of service providers trained to use an EBT for HIV prevention ever attempt to implement that treatment, and only half of those providers implement the treatment with fidelity (Collins et al., 2007). The consistency of such findings outside of laboratory clinics, regardless of the specific treatment being evaluated, suggest the additional need for routine feedback on how the clinician is implementing the treatment, even if only at the level of adherence to basic treatment elements. This is yet another knowledge management issue. For a therapist's future actions to be guided by useful information, we need better methods to deliver that information, whether it comes from the literature, the client's response, or the therapist's own past actions (Daleiden \& Chorpita, 2005).

\section{Conclusion}

We believe Kazdin and Blase (2011) have identified a major failure in knowledge management. If, however, the field interprets this challenge as a failure in knowledge production, we will continue in our old habits of promulgating EBTs that few with mental health needs may ever encounter. The current national economic condition suggests that we should not spend all of our time or other resources solely on producing more treatments that are only incrementally better. We also need new paradigms.

It is time to develop models that allow for designing treatments across laboratories, across disciplines, and across researchers and practitioners. We encourage researchers and treatment developers to consider packaging and studying their new treatments in discrete treatment units or modules that can "plug and play" with those of other developers. We may find that common practice elements, features, processes, or functions are robust across a wide variety of delivery platforms or workforces. Meanwhile, we encourage practitioners - broadly defined - to be open to using those treatment elements or modules, and to see them as supports for making their current work more effective. Practitioners will also need to help researchers understand how much of that support is enough, too little, or too much. As we all continue to learn more about how to alleviate mental illness, we must keep in mind that what we know is irrelevant when separated from the question of what to do with what we know.

\section{Declaration of Conflicting Interests}

The author(s)declared no potential conflicts of interest with respect to the research, authorship, and/or publication of this article.

\section{Funding}

The author(s) received no financial support for the research, authorship, and/or publication of this article.

\section{References}

Addis, M. E., \& Krasnow, A. D. (2000). A national survey of practicing psychologists' attitudes toward psychotherapy treatment manuals. Journal of Consulting and Clinical Psychology, 68, 331-339.

Allen, L. A., McHugh, R. K., \& Barlow, D. H. (2008). Emotional disorders: A unified protocol. In D. H. Barlow (Ed.), Clinical handbook of psychological disorders (4th ed., pp. 216-249). New York, NY: Guilford Press.

APA Presidential Task Force. (2006). Evidence-based practice in psychology. American Psychologist, 61, 271-285.

Barth, R. P., Lee, B. R., Lindsey, M. A., Collins, K. S., Strieder, F., Chorpita, B. F., . . . Sparks, J. R. (in press). Evidence-based practice at a crossroads: The timely emergence of common elements and common factors. Research on Social Work Practice.

Bickman, L. (2008). A measurement feedback system (MFS) is necessary to improve mental health outcomes. Journal of the American Academy of Child \& Adolescent Psychiatry, 47, 1114-1119.

Borntrager, C., Chorpita, B. F., Higa, C., \& Weisz, J. R. (2009) Provider attitudes toward evidence-based practices: Are the concerns with the evidence or with the manuals? Psychiatric Services, 60, 1-5.

Chambers, D. A., Ringeisen, H., \& Hickman, E. E. (2005). Federal, state, and foundation initiatives around evidence-based practices for child and adolescent mental health. Child and Adolescent Psychiatric Clinics of North America, 14, 307-327.

Chambless, D. L. \& Hollon, S. D. (1998). Defining empirically supported therapies. Journal of Consulting and Clinical Psychology, 66, 7-18.

Chorpita, B. F., Bernstein, A., \& Daleiden, E. L. (in press). Empirically guided coordination of multiple evidence-based treatments: An illustration of relevance mapping in children's mental health services. Journal of Consulting and Clinical Psychology.

Chorpita, B. F., \& Daleiden, E. L. (2009). Mapping evidence-based treatments for children and adolescents: Application of the distillation and matching model to 615 treatments from 322 randomized trials. Journal of Consulting and Clinical Psychology, 77, $566-579$.

Chorpita, B. F., \& Daleiden, E. L. (2010). Building evidence-based systems in children's mental health. In A. E. Kazdin \& J. R. Weisz (Eds.), Evidence-based psychotherapies for children and adolescents (pp. 482-499). New York, NY: Oxford Press. 
Chorpita, B. F., \& Weisz, J. R. (2009). MATCH-ADTC: Modular approach to therapy for children with anxiety, depression, trauma, or conduct problems. Satellite Beach, FL: PracticeWise.

Collins, C., Phields, M. E., \& Duncan, T., \& Science Application Team. (2007). An agency capacity model to facilitate implementation of evidence-based behavioral interventions by communitybased organizations. Journal of Public Health Management and Practice[Suppl.], S16-S23.

Daleiden, E., \& Chorpita, B. F. (2005). From data to wisdom: Quality improvement strategies supporting large-scale implementation of evidence based services. Child and Adolescent Psychiatric Clinics of North America, 14, 329-349.

Graham, I. D., Logan, J., Harrison, M. B., Straus, S. E., Tetroe, J., Caswell, W., \& Robinson, N. (2006). Lost in knowledge translation: Time for a map? Journal of Continuing Education in the Health Professions, 26, 13-24.

Hawe, P., Shiell, A., \& Riley, T. (2004). Complex interventions: How "out of control" can a randomized controlled trial be? British Medical Journal, 328, 1561-1563.

Ingram, B. L., Flannery, D., Elkavich, A., \& Rotheram-Borus, M. J. (2008). Common processes in evidence-based adolescent HIV prevention programs. AIDS and Behavior, 12, 374-383

Kazdin, A. E. (2003). Research design in clinical psychology (4th ed.). Boston, MA: Allyn \& Bacon.

Kazdin, A. E. (2008a). Evidence-based treatments and delivery of psychological services: Shifting our emphases to increase impact. Psychological Services, 5, 201-215.
Kazdin, A. E. (2008b). Evidence-based treatment and practice: New opportunities to bridge clinical research and practice, enhance the knowledge base, and improve patient care. American Psychologist, 63, 146-159.

Kazdin, A. E., \& Blase, S. L. (2011). Rebooting psychotherapy research and practice to reduce the burden of mental illness. Perspectives on Psychological Science, 6, 21-37.

Lambert, M. J., Harmon, C., Slade, K., Whipple, J., \& Hawkins, E. (2005). Providing feedback to psychotherapists on their patients' progress: Clinical results and practice suggestions. Journal of Clinical Psychology, 61, 165-174.

Layne, C. M., Ghosh Ippen, C., Strand, V., Stuber, M., Abramovitz, R., Reyes, G., .. . Pynoos, R. (in press). The core curriculum on childhood trauma: A tool for training a trauma-informed workforce. Psychological Trauma: Theory, Research, Practice, and Policy.

Rotheram-Borus, M. J., Ingram, B. L., Swendeman, D., \& Flannery, D. (2009). Common principles embedded in effective adolescent HIV prevention programs. AIDS and Behavior, 13, 387-398.

Schoenwald, S. K., Garland, A. F., Southam-Gerow, M. A., Chorpita, B. F., \& Chapman, J. E. (2011). Adherence measurement in treatments for disruptive behavior disorders: Pursuing clear vision through varied lenses. Manuscript submitted for publication.

U.S. Department of Health and Human Services. (2007). U.S. Department of Health and Human Services Strategic Plan. Washington, DC: Author. 


\title{
Delivery Systems Can Determine Therapy Cost, and Effectiveness, More Than Type of Therapy
}

Perspectives on Psychological Science 6(5) 498-502

(C) The Author(s) 2011

Reprints and permission:

sagepub.com/journalsPermissions.nav DOI: 10.1 177/1745691611416994

http://pps.sagepub.com

(S)SAGE

\author{
Brian T. Yates \\ Department of Psychology, American University, Washington, DC
}

\begin{abstract}
We should go further than Kazdin and Blase (20II) in emphasizing the importance of the costs and effectiveness of alternative delivery systems for therapies. I propose that the manner in which therapy is delivered often determines its cost, and its effectiveness, more than the type of therapy delivered. In this article, I illustrate this argument through compiled research and describe several inexpensive delivery systems with the aid of metaphors.
\end{abstract}

\section{Keywords}

therapy, cost, delivery system, cost-effectiveness, benefit, scientist-manager-practitioner

In their article "Rebooting Psychotherapy Research and Practice to Reduce the Burden of Mental Illness," Kazdin and Blase (2011) praised our progress in developing efficacious techniques for treating a variety of severe and costly psychological problems, but they also noted the profound ineffectiveness of current methods for delivering these techniques to produce socially significant reductions in mental illness and in the costs of mental illness to society.

Our focus over the past century has been, perhaps necessarily, on developing psychological techniques that work most of the time for most people for several important psychological problems. The promise of better living through psychological technologies developed through systematic scientific inquiry has yet to be fulfilled, however. We have come only halfway at best. To a limited extent, we have the knowledge to cure and enhance ourselves psychologically in a number of areas, but we have not found ways to use this knowledge to help most of the people most of the time for their most serious psychological dysfunctions. It is as if the techniques or tools for fixing important problems were resting in locked toolboxes, shown to one person at a time with brief instruction on tool use, rented at rather high hourly rates for a few weeks, and then locked back in the toolbox. If universities offered education via similar means, most instruction would be independent studies taught by tenured full professors for an hour or two per week, to 5 to 10 individual students daily, with small amounts of reading that kept key knowledge accessible only to the professors - and without any course evaluation by the few students being taught!

Among the solutions to problems we now face in delivering our treatment technologies to those who need them the most is the development, testing, and refinement of more effective methods of delivering treatment-methods that use less therapist and client time, minimize client transportation costs as well as brick-and-mortar space, and use less of other increasingly scarce and costly resources. Just as therapy is no longer an art but a science based on research evidence gathered in clinical settings, so too can be its delivery. Research of this sort is not particularly popular with most graduate students, funders, or rank and tenure committees. In my experience, it is criticized as secondary in importance, mundane to conduct, or too site- or therapist-specific to be of use to the field. Similar arguments were made decades ago against the desirability of conducting research on the cost effectiveness of different therapeutic technologies (e.g., Strupp, 1981), yet this sort of work has become popular at least in what is called for, if not in what is often performed, in applied psychology (cf. American Psychological Association Presidential Task Force on Evidence Based Practice, 2006).

Research on less costly and more effective ways to deliver therapy is what we need, so that we can use evidence-based delivery systems to provide evidence-based services to the most people for the least necessary expenditure of resources per person (Yates, 1980, 1994). This sort of research is only beginning to be conducted in a thorough, systematic manner that includes careful measurement of costs, and effectiveness,

\section{Corresponding Author:}

Brian T. Yates, Department of Psychology, American University, 4400 Massachusetts Avenue, NW, Washington, DC 20016-8062

E-mail: byates@american.edu 
from multiple perspectives (cf. Tate, Finkelstein, Khavjou, \& Gustafson, 2009).

\section{Delivery Systems for Therapy: Sieves, Golden Ladles, or Plastic Spoons?}

The delivery system used to provide a therapy is, arguably, a stronger potential determinant of the effectiveness and cost of that therapy than the effectiveness and costs of specific techniques used in the therapy. Consider the common plastic spoon as a metaphor for the delivery system for the "medicine" of therapy, with the ingredients of the medicine being the specific techniques that are carefully combined by the practitioner to help a client with a particular problem. Suppose the practitioner has studied research regarding which combinations of ingredients work best for this sort of client presenting this particular problem. The ingredients most likely to be effective are chosen. Perhaps the therapist even considers the expense of those ingredients. For example, the therapist might decide whether to prescribe time-consuming hourly recording of catastrophizing and self-negating cognitions, or a simpler and quicker daily check-off log for occurrence of catastrophizing and self-negating cognitions. The therapist proceeds to select the ingredients that fulfill the requirements of best evidencebased practices and that minimize client resources consumed. Having identified and optimized an evidence-based amalgam of techniques, should the practitioner "pour" this carefully developed mixture into ...

- ... a sieve, from which the medicine largely dissipates before it reaches the client?

- ... an exquisite golden ladle, which delivers the exact combination of ingredients to the client with high fidelity but at unnecessary cost? or

- ... a plastic spoon, with sufficient integrity to deliver the medicine at the minimum necessary expense?

Clearly, the "plastic spoon" delivery system is what most would select as the optimally effective and least costly delivery system for most clients. I believe that we have the right medicine but are using golden ladles to deliver that medicine, which prevents it from getting to most people - particularly to those who need it the most and can least afford it.

Research comparing delivery systems that promise to transmit most or all of the potential effectiveness of a psychological technique while using fewer resources (and costing less) has begun, particularly for problems related to physical health (cf. Ritterband \& Tate, 2009). The variety of potential "plastic spoons" researched to date includes Internet-based interventions addressing everything from social anxiety and panic disorder to eating disorders, automated phone interventions teaching self-management of exercise to diabetics (Handley, Shumway, $\&$ Schillinger, 2008), and video-based motivational and cognitive-behavioral interventions for HIV risk reduction in females in military service (Essien et al., 2011).

\section{Differences in Therapy Cost Versus Differences in Therapy Effectiveness}

How much of a difference can a delivery system make in the effectiveness or cost of a therapy? Meta-analyses of randomized clinical trials of a wide variety of therapeutic techniques have shown repeatedly that many therapy techniques work, and do so reasonably if not similarly well, for some psychological problems (e.g., Shadish, Matt, Navarro, \& Phillips, 2000; Smith, Glass, \& Miller, 1980). Rigor of design, training of practitioners, and other variables have been examined in these analyses. The consensus is clear: Therapy works, pretty well, most of the time for most people and a variety of problems. With several notable exceptions (cf. Siev, Huppert, \& Chambless, 2009), different therapies can be surprisingly similar in their effectiveness, depending on several factors, including characteristics of the therapist and other components of the therapeutic delivery system. Most are better than no therapy, measurement and attention controls, or placebo therapies (cf. Smith et al., 1980). Almost all of these studies use one-on-one therapies, however: golden ladle delivery systems!

Research on the effectiveness of different means of providing the same therapeutic techniques remains, unfortunately, rare. What research there is on delivery systems suggests that considerable savings could be achieved with little or no reduction in therapy outcomes if a "plastic spoon" delivery system was utilized. A substantial research literature finds, for example, little evidence for the incremental effectiveness of using doctoral rather than trained paraprofessional therapists to deliver therapy techniques for a wide range of psychological problems (cf. reviews by Berman \& Norton, 1985; Durlak, 1979; Smith et al., 1980, and more recently Shadish et al., 2000).

Other research demonstrates that combinations of different therapeutic agents, as well as variations in other aspects of treatment provision, can have profound effects on the cost, if not the effectiveness, of therapy. For example, overweight clients assigned to two weight-loss treatments lost statistically similar amounts of excess adipose tissue, but at an average cost of $\$ 44.60$ versus $\$ 3.00$ per $1 \%$ reduction in excess weight (Yates, 1978)! (Note that these cost-effectiveness ratios were in 1976 dollars.) This difference in cost was accounted for largely by the former treatment's use of highly paid staff meeting clients several days weekly for a standard number of weeks in prestigious offices. In the latter treatment, former clients implemented a program detailed in manuals for groups of clients who met in plain and often donated space and who paid per session attended.

Similarly, Siegert and Yates (1980) randomly assigned parents to one of three systems for delivering the same behavioral training for managing disruptive behaviors of their children, or to a measurement and attention control condition. All three training systems produced strong and statistically similar improvements in behaviors targeted by the parents. All three training systems required different mixtures of different types 
of resources. The individual in-office delivery system required clients to participate in traditional one-on-one sessions for child management training in a therapist's office. The group in-office delivery system had clients participate in group training sessions in therapist offices. The individual in-home delivery system had therapists train clients in clients' homes. Depending on whether client time and client transportation resources were included in cost calculations, the individual inoffice delivery system was substantially more expensive than the individual in-home delivery system and often more than the group in-office delivery system as well.

Similar research using random assignment of 1,827 severely disturbed adults to referral or nonreferral to consumeroperated services (COS) found little difference between multiple sites and techniques in COS effectiveness. Profound differences were observed, however, in the amounts of monetary as well as donated resources consumed by delivery of COS services to individual clients (Yates et al., in press).

\section{Research on Effectiveness and Costs of Delivery Systems Can Save Resources}

An example of how different delivery systems can affect treatment effectiveness as well as treatment costs is provided by a slight reinterpretation of a randomized clinical trial reported by Bandura, Blanchard, and Ritter (1969). Snake-phobic participants were assigned randomly to either (a) a measurement control condition, (b) systematic desensitization, (c) modeling of successively more anxiety-provoking interactions with snakes delivered by a film that participants could pause or reverse, or (d) modeling of progressive snake approach by a paraprofessional model. Bandura et al. did not entirely control time spent in each condition, allowing it to vary as long as it did not exceed $5.25 \mathrm{hr}$. Resources common to all treatments conditions were office space, advertising for research participants, and clients' own transportation expenses.

Bandura et al. (1969) found that the live delivery system for modeling techniques of snake phobia reduction allowed $92 \%$ of participants to achieve the "terminal" step of sitting for $2 \mathrm{~min}$ with their hands at their sides and a four-foot nonpoisonous snake in their laps. This combination of delivery system and technique was found to consume surprisingly few temporal resources: an average 2.17 practitioner hours and a similar number of client hours in direct service. Snake approach modeling via film allowed $33 \%$ of participants to achieve the same terminal step, requiring a mean $2.77 \mathrm{hr}$ from clients plus a few minutes of a paraprofessional's time to show clients how to operate the film projector. The measurement control delivery system was inexpensive but had no effect whatsoever on snake approach. Both live and film delivery systems for the modeling technique were superior in effectiveness, and they consumed substantially less provider and client time than the mean $4.53 \mathrm{hr}$ consumed for clients who were delivered the usual technique of systematic desensitization (which enabled only $25 \%$ of clients to reach the terminal step in snake approach).
In sum, Bandura et al. demonstrated that inexpensive combinations of therapy techniques and delivery systems (i.e., modeling delivered via client-controlled film projection) could be significantly more effective than traditional delivery systems (such as the one-on-one in-office provision of technologies such as systematic desensitization). Newer information technologies could enable even greater cost savings. The film showing the snake interaction models, for instance, now could be offered at near-zero cost in transportation and computer resources by streaming Internet video directly to clients' smartphones, and not necessarily in therapist offices, possibly with similar effectiveness.

Bandura et al.'s findings also show that, for a small increment in resources (i.e., an average $0.6 \mathrm{hr}$ of client time, plus perhaps $2 \mathrm{hr}$ of provider time) and an evidence-based choice of treatment technology (i.e., modeling as opposed to systematic desensitization), the effectiveness of therapy for achieving a rather complete "cure" can be increased from an average $33 \%$ to $92 \%$ of clients. This is the sort of information that, when provided on a larger scale for a variety of therapeutic techniques for the wider range of delivery systems now available, could provide therapists with evidence on how to provide treatment both effectively and inexpensively.

\section{Monetary Benefits of Delivery Systems Need to Be Measured, Too}

While adjusting our research to examine the relative effectiveness of different delivery systems for therapies according to traditional psychological measures, we also might include among our measures client reports and other indices of how their productivity and income were affected by therapy and how their use of health and criminal justice services may have declined. These are the types of monetary measures that can be contrasted to the costs of providing therapy through one delivery system or another to determine which combinations of therapeutic techniques and delivery systems are most cost beneficial (i.e., which pay for themselves soonest and most fully; cf. Yates, 2005). Third-party funders will likely support only those combinations of technique and delivery system that return their investments most quickly and enduringly. Once this is shown, that combination may be widely implemented as it would be readily reimbursed.

\section{Additional Suggestions for More Cost- Effective Delivery Systems}

Many therapists will note that issues of client confidentiality and the need for privacy may prevent some delivery systems, such as group therapy, from being used for some clients. That does not mean that one-on-one, face-to-face, breathing-thesame-air interaction is required for effective delivery of therapy. Video and audio links are widely available at low cost to anyone with even temporary access to a smartphone, a tablet, or a computer and can be kept confidential and possibly 
anonymous. When integrated with Web-based, e-mailed, or downloaded manuals and worksheets, plus videos illustrating how various psychological techniques can be applied, widescale administration of a variety of therapeutic techniques seems both possible and affordable for most rather than some people. For example, Mihalopoulos, Vos, Pirkis, Smit, and Carter (2011) found that both bibliotherapy and group therapy were effective delivery systems for preventing depression, with bibliotherapy providing more than twice the impact per dollar invested.

The idea of integrating evidence-based techniques of therapy with means of delivering treatment services that have themselves been shown to be both effective and not inherently expensive is not particularly new (cf. Yates, 1995), and yet is only beginning to take hold. Some psychological practices could emulate the delivery system used by some dentists in private practice, who see the costs and evidence of success in their monthly accounting records and patient rolls. Technologies for preventing and treating dental problems are, perhaps, no less inherently expensive than are psychological technologies. Moreover, many dentists continue to focus on one client at a time as many therapists wish to continue to do. Often these dental techniques are delivered literally face-to-face. Other services dictated by these decisions are performed by paraprofessionals trained and supervised by the dentist. In one-dentist practices, a receptionist makes appointments, greets patients, manages the office, submits bills, and accepts payments. A dental assistant interviews new and returning patients, periodically updates patient medical records, and takes X-rays as needed according to a schedule determined by the dentist. Technologies not requiring staff time may be used as well. A looping video viewable as I wait for X-ray results informs me about the latest cosmetic procedures available, but also could remind me about the best way to floss. My dentist cleans and inspects my teeth, but could avoid the former activity if he did not so relish discussing his latest motorcycle exploits, or the cost of college tuition for his daughter, without having me talking back. He even provides me with a cognitive-behavioral intervention of sorts that I regularly self-administer and suggest to others: "Only floss the teeth you want to keep!"

A high-resolution paper display, aka "chart," on the wall of the examining room informs me about root decay and root canal procedures, providing further motivation for preventive self-management cognitions and behaviors. The receptionist schedules the next appointment. Total time in the office: about $45 \mathrm{~min}$. Total dentist time directly serving me: 5 to $10 \mathrm{~min}$. The result is a substantial savings of his time and my monetary resources, relative to what I would pay if he performed all of the above services (as do many therapists, I have learned). He has two or three patients at various stages of service delivery at any one time. We all feel attended to and appreciated. He gets us in, gets us out, and we receive high-quality treatment, at low cost, due to the use of paraprofessionals, videos, and biblio (wall chart) devices. My health maintenance organization (HMO) delivers other medical services with similar combinations of lay, paraprofessional, and professional staff, all making optimal contributions to service delivery.

Some will take unintended offense at the comparison between dental services, HMOs, and mental health services. I apologize! I do not mean to demean mental health services, or dental or other health services for that matter. Many service systems function similarly, from ophthalmology to general practice to vehicle maintenance, with work distributed among staff and display media according to their abilities. Similar service systems using a mixture of staff with varying levels of expertise have been developed and implemented for some time to deliver particular mental health services (e.g., Tharp \& Wetzel, 1969), thus meeting needs for student training as well as treatment for communities of clients. Some have achieved notable commercial success and have been funded by major health service systems (e.g., Cummings, O’Donohue, \& Ferguson, 2002). Arguably, externships, if not internships, provide some paraprofessional service delivery as well in mental health contexts, albeit often within the same one-on-one delivery system.

To conduct and apply delivery systems research, doctoral training models for psychologists who would become scientist-manager-practitioners have been proposed (e.g., DeMuth, Yates, \& Coates, 1984). We now need to implement these models and these delivery systems and examine their effectiveness, costs, and benefits with the same research methodologies we used to maximize the effectiveness of treatment techniques. The people we serve, and who ultimately fund our treatment and research, expect and deserve no less.

\section{Declaration of Conflicting Interests}

The author declared no potential conflicts of interest with respect to the authorship or the publication of this article.

\section{References}

American Psychological Association Presidential Task Force on Evidence Based Practice. (2006). Evidence-based practice in psychology. American Psychologist, 61, 271-285.

Bandura, A., Blanchard, E. B., \& Ritter, B. (1969). Relative efficacy of desensitization and modeling approaches for inducing behavioral, affective, and attitudinal changes. Journal of Personality and Social Psychology, 13, 173-199.

Berman, J. S., \& Norton, N. C. (1985). Does professional training make a therapist more effective? Psychological Bulletin, 98, 401-406.

Cummings, N. A., O’Donohue, W. T., \& Ferguson, K. E. (2002). The impact of medical cost offset on practice and research: Making it work for you. Reno, NV: Context Press.

DeMuth, N. M., Yates, B. T., \& Coates, T. (1984). Psychologists as managers: Old guilts, innovative applications, and pathways to being an effective managerial psychologist. Professional Psychology, 15, 758-768.

Durlak, J. A. (1979). Comparative effectiveness of paraprofessional and professional helpers. Psychological Bulletin, 86, 80-92.

Essien, E. J., Mgbere, O., Monjok, E., Ekong, E., Holstad, M. M., \& Kalichman, S. C. (2011). Effectiveness of a video-based motivational 
skills-building HIV risk-reduction intervention for female military personnel. Social Science \& Medicine, 72, 63-71. doi:10.1016/ j.socscimed.2010.10.012

Handley, M. A., Shumway, M., \& Shillinger, D. (2008). Costeffectiveness of automated telephone self-management support with nurse care management among patients with diabetes. Annals of Family Medicine, 6, 512-518. doi:10.1370/afm.889

Kazdin, A. E., \& Blase, S. L. (2011). Rebooting psychotherapy research and practice to reduce the burden of mental illness. Perspectives on Psychological Science, 6, 21-37.

Mihalopoulos, C., Vos, T., Pirkis, J., Smit, F., \& Carter, R. (2011). Do indicated preventive interventions for depression represent good value for money? Australian and New Zealand Journal of Psychiatry, 45, 36-44. doi:10.3109/00048674.2010.501024

Ritterband, L. M., \& Tate, D. F. (2009). The science of Internet interventions. Annals of Behavioral Medicine, 38, 1-3. doi:10.1007/ s12160-009-9132-5

Shadish, W. R., Matt, G. E., Navarro, A. M., \& Phillips, G. (2000). The effects of psychological therapies under clinically representative conditions: A meta-analysis. Psychological Bulletin, 126, 512-529.

Siegert, F. A., \& Yates, B. T. (1980). Cost-effectiveness of individual in-office, individual in-home, and group delivery systems for behavioral child-management. Evaluation and the Health Professions, 3, 123-152.

Siev, J., Huppert, J. D., \& Chambless, D. L. (2009). The Dodo Bird, treatment technique, and disseminating empirically supported treatments. Behavior Therapist, 32, 69-76.
Smith, M. L., Glass, G. V., \& Miller, T. I. (1980). The benefits of psychotherapy. Baltimore, MD: Johns Hopkins University Press.

Strupp, H. (1981). Psychotherapy: The question of evidence. Monitor on Psychology, 12(3), 2.

Tate, D. F., Finkelstein, E. A., Khavjou, O., \& Gustafson, A. (2009). Cost effectiveness of Internet interventions: Review and recommendations. Annals of Behavioral Medicine, 38, 40-45. doi:10.1007/s12160-009-9131-6

Tharp, R. G., \& Wetzel, R. J. (1969). Behavior modification in the natural environment. New York, NY: Academic Press.

Yates, B. T. (1978). Improving the cost-effectiveness of obesity programs: Reducing the cost per pound. International Journal of Obesity, 2, 249-266.

Yates, B. T. (1980). Improving effectiveness and reducing costs in mental health. Springfield, IL: Thomas.

Yates, B. T. (1994). Toward the incorporation of costs, costeffectiveness analysis, and cost-benefit analysis into clinical research. Journal of Consulting and Clinical Psychology, 62, 729-736.

Yates, B. T. (1995). Cost-effectiveness analysis, cost-benefit analysis, and beyond: Evolving models for the scientist-manager-practitioner. Clinical Psychology: Science and Practice, 2, 385-398.

Yates, B. T. (2005). Cost-effectiveness analysis and cost-benefit analysis. In D. DuBois \& M. Karcher (Eds.), Handbook for youth mentoring (pp. 525-545). Thousand Oaks, CA: Sage.

Yates, B. T., Mannix, D., Freed, M. C., Campbell, J., Johnsen, M., Jones, K., \& Blyler, C. R. (in press). Consumer-operated service programs: Monetary and donated costs and cost-effectiveness. Psychiatric Rehabilitation Journal. 


\title{
Reducing the Burden of Mental Illness in Military Veterans: Commentary on Kazdin and Blase (20 I I)
}

Perspectives on Psychological Science 6(5) 503-506

(C) The Author(s) 2011

Reprints and permission:

sagepub.com/journalsPermissions.nav DOI: $10.1177 / 1745691611416995$

http://pps.sagepub.com

(S)SAGE

\author{
Denise M. Sloan, Brian P. Marx, and Terence M. Keane \\ National Center for PTSD, VA Boston Healthcare System, and Boston University School of Medicine, Boston, MA
}

\begin{abstract}
Clinical psychology as a profession owes much to the recognition of the psychosocial needs of servicemen and women returning from World War II and the Korean conflict. The current conflicts in Iraq and Afghanistan represent another opportunity for substantial advancements in assessment and treatment practices. Stimulated by the prescient article by Kazdin and Blase (20II), we briefly describe innovations in evidence-based practices currently being implemented in the Veterans Health Administration to best serve the more than 2 million returning servicemen and women. The largest healthcare system in the nation, the U.S. Department of Veterans Affairs began a wide range of innovations early this century to include dissemination of evidence-based mental health treatments, the use of anonymous Internet-based interventions to reach large numbers of military personnel who may not otherwise present for mental health service, the use of videoconferencing to deliver assessment and treatment to individuals residing in remote locations, and the use of laypersons (e.g., peers) for treatment delivery. In addition to describing the strengths of these efforts to reduce mental health burden, we also discuss persisting barriers and limitations of these innovative efforts within this system of healthcare.
\end{abstract}

\section{Keywords}

dissemination, implementation, telehealth

Recently, Kazdin and Blase (2011) described the substantial burden of mental illness experienced by many individuals and sounded a clarion call to develop alternative methods of delivering psychotherapy to reduce this burden. The U.S. Department of Veterans Affairs (VA) has already developed and implemented novel programs to address the mental health needs and associated burdens of all its veterans. In this commentary, we pay particular attention to how both VA and the Department of Defense (DoD) are utilizing technology to improve access to care, the initiatives in place to foster lay and peer counseling in order to deliver care to a greater number of veterans and service men and women, and how the VA is disseminating and implementing evidence-based treatments (EBTs) for posttraumatic stress disorder (PTSD) to provide veterans with the best possible mental health care. We also describe ongoing challenges to meeting the mental healthcare demands of a large number of veterans in need of these services.

The VA operates an internationally recognized network of 147 medical centers, 292 Vet Centers, and 642 CommunityBased Outpatient Clinics. These facilities provide mental health care services to almost 1.9 million veterans. As such, the VA is the largest provider of mental health care services in the United States. Care for the returning combat deployed
Operation Enduring Freedom and Operation Iraqi Freedom (OEF/ OIF) veterans is among the highest priorities in the VA's mental healthcare system, as it is estimated that over one third of all OEF/OIF veterans have a mental health condition and this number continues to rise (e.g., Hoge, Auchterlonie, \& Milliken, 2006).

In 2004, responding to the high prevalence of mental health conditions among its constituents, the VA developed a Mental Health Strategic Plan (MHSP) rooted in the government-wide President's New Freedom Commission Report to address a growing population of veterans with unmet mental health care needs. The goal for this strategic plan is to reduce the burden of mental illness by reducing stigma; promoting recovery; ensuring equal access and reducing variability of care; providing culturally competent care to veterans of all ages, races, ethnic groups and genders; being veteran and family centered; ensuring collaborative care models are used in primary-care team structure; and employing evidence-based population approaches. With the continuing leadership of Dr. Antonette Zeiss (Zeiss \&

\section{Corresponding Author:}

Denise M. Sloan,VA Boston Healthcare System, I50 S. Huntington Avenue, Boston 02130

E-mail: denise.sloan@va.gov 
Karlin, 2011), many initiatives have been launched over the past several years to meet the goals of VA's 2004 MHSP.

Kazdin and Blase (2011) suggested the adaptation of technology to deliver psychotherapy; the VA has recognized that telehealth technologies (e.g., web-based, phone, video teleconference) are an increasingly important method of ensuring equal and timely access to mental health care services to patients who would otherwise not have such access to services because they live in locations that are a considerable distance from the nearest VA facility (Sloan, Gallagher, Feinstein, Lee, $\&$ Pruneau, 2011). Another advantage of telehealth technology is its ability to provide mental healthcare services quickly and efficiently to large numbers of individuals. Further, these methods may also be used to connect with many veterans who perceive stigma associated with their conditions and would not be inclined to present themselves in person for therapy (Pietrzak, Johnson, Goldstein, Malley, \& Southwick, 2009).

Both the VA and DoD are funding research to examine the viability of these new technologies for the delivery of EBTs, including the use of videoconferencing to deliver group-based cognitive-behavioral treatments (Morland et al., 2010) and prolonged exposure therapy (PE; Tuerk, Yoder, Ruggiero, Gros, \& Acierno, 2010) and an internet-based treatment to deliver mental healthcare treatment anonymously to military service men and women (Litz, Engel, Bryant, \& Papa, 2007). Smartphone applications are also being developed to enhance existing care and provide self-help treatment to veterans and military service personnel who may be reluctant or unable to present for treatment services. For example, VA Secretary Shinseki recently announced that the VA and DoD have launched the PTSD Coach mobile smartphone application, a therapy augmenting tool that provides information on EBTs for PTSD, tools for screening and symptom tracking, skills for symptom management, and direct links to support. PTSD Coach is the first in a suite of VA and DoD jointly developed mobile smartphone applications that will cover a range of mental health conditions.

Communication between patient and provider and the provision of psycho-education are critical features of health care in managing chronic conditions of all types. The website www. myhealthevet.com provides access to important information about a veteran's medical care, access to components of their medical records, the ability to make medical appointments, and the availability of wellness resources such as psycho-educational materials and self-screening instructions. Additional features will be added to this Internet-based website, which was designed to support the VA recovery model of mental health care.

For different purposes, DoD, in collaboration with the VA National Center for PTSD, has also created a website, www .afterdelopyment.org, to deliver wellness resources to service members, veterans, and their families. These websites allow veterans and active duty personnel to play a vital role in their own health care and to be able to access needed resources quickly and efficiently. Although these telehealth technology initiatives are breaking new ground in providing mental health care services to a larger number of veterans and service members in need of care, these approaches still may not reach all individuals in need of care, particularly individuals from a low socioeconomic background, lower education level, and/or individuals residing in rural communities where broadband internet access is less common (Smith, 2010).

Consistent with Kazdin and Blase's (2011) suggestion, the VA and DoD are engaged in several nationwide programs in which lay individuals and recovered peers provide mental healthcare services. One example of such a program is combat and operational stress first aid (COSFA; Nash, Krantz, Stein, Westphal, \& Litz, in press). A significant barrier to seeking mental health care services among servicemen and women is confidentiality concerns. Services delivered from a chaplain remain confidential within the military; thus, servicemen and women may be more likely to disclose mental health concerns to chaplains. The COSFA program has trained chaplains to deliver brief psychologically based interventions to military servicemen and women, and the program appears to be effective in terms of increasing the number of military personnel seeking mental health care services. Innovative programs are also underway to train VA chaplains and VA police; these individuals represent VA employees with whom veterans have frequent contacts and can provide a gateway to more intensive treatment delivery when needed.

Nearly a decade ago, the VA leadership in mental healthcare realized that EBTs were inconsistently available across this national system of care. Although not specifically recommended in their article, we suspect Kazdin and Blase would support the dissemination initiatives that began in the VA 5 years ago. Despite the substantial advances made in the development and evaluation of psychological treatments, mental health care providers had not yet incorporated EBTs into routine clinical practice (Kazdin, 2008). The lack of dissemination and implementation of EBTs represents a substantial hurdle in the delivery of effective treatments and, in turn, is a major barrier in reducing the nation's mental health care burden. The infrequent use of EBTs was noted within the VA itself (Rosen et al., 2004). As the largest mental healthcare system in the United States, and the largest provider of PTSD treatment in the world, the VA is in a unique position to disseminate EBTs to mental healthcare providers in the interest of providing the best care possible to veterans seeking psychological care, whether in Boston or in rural Montana. As part of a national mandate that all veterans have access to EBTs, the VA has implemented multiple national initiatives to disseminate and implement EBTs. This effort started with disseminating PTSD EBTs of cognitive processing therapy (CPT) and PE throughout the VA health care system.

This ongoing national initiative involves a multilevel process in which VA providers receive intensive, standardized, and competency-based training in the delivery of CPT and PE (Karlin et al., 2010). Following intensive training workshops, the providers continue to receive consultation and peer support by local VA expert providers. These local expert providers 
have received intensive, standardized training by experts in either CPT or PE, and they must pass a certification process to serve in as a VA local expert provider. In turn, VA providers must pass a certification process for CPT and/or PE to be qualified to deliver these treatments to veterans. In addition to this credentialing process, all VA providers have access to an electronic VA intranet site that provides core and supplemental training materials, a discussion board, and other provider-oriented materials. The intranet provides ongoing consultation to enhance education and provide support services as well as further ensuring CPT and PE treatments are being effectively implemented by the providers.

As others have noted (e.g., Kazdin, 2008), although mental healthcare providers may be trained in EBTs, they may not successfully implement these treatments. Successful implementation of EBTs is as critical as dissemination in reducing the mental healthcare burden. Recognizing the importance of implementation, the VA has instituted multiple national initiatives to promote implementation of CPT and PE. For example, the newly launched PTSD National Mentoring Program promotes regional and national communication between PTSD clinical managers and the sharing of best practices to clinic design and care processing. In addition, a PTSD consultation program has been established in which VA providers can receive direct consultation on PTSD assessment and treatment from PTSD expert clinical psychologists and physicians. The intranet site that contains the discussion board and provideroriented materials also promotes implementation of PE and CPT. Providing a locally trained expert in CPT and PE further promotes that these treatments will be implemented. As others have noted (e.g., Cook, Biyanova, \& Coyne, 2009; Cook, Schnurr, Biyanova, \& Coyne, 2009), having a local provider implementing EBTs and available for peer supervision appears to be one effective approach to have other providers adopt evidence-based practices.

Taken together, these combined dissemination and implementation initiatives have resulted in nearly all VA facilities providing evidence-based PTSD treatment to veterans, and over 4,400 VA and DoD mental healthcare providers are now trained in CPT and/or PE (Karlin et al., 2010). The VA has also instituted treatment outcome monitoring within the VA to evaluate the success of PTSD EBT dissemination and implementation efforts - an approach that is essential to examining the effectiveness of these efforts. It is too early to evaluate whether or not these major national dissemination and implementation efforts are successful in reducing PTSD symptom severity and functional impairment among veterans. However, it is clear that many mental health care providers are now trained in CPT and PE and that these treatments are being implemented to veterans in need of treatment services. Effectiveness studies are now needed.

Building on the success of the PTSD EBT dissemination and implementation programs, there are additional dissemination and implementation EBT initiatives currently underway that address other mental health problems frequently seen within the veteran population (e.g., depression, serious mental illness, and substance abuse). The VA national initiatives to disseminate and implement EBTs in a standardized fashion can serve as a model for the field in how to successfully train large numbers of mental healthcare providers in EBTs and how to effectively implement these EBTs in practice.

Although the VA and DoD have launched many initiatives in recent years with the overall goal of ensuring that veterans have access to the highest quality of care, there are some remaining challenges and limitations. Perhaps the most significant challenge is the actual implementation of these programs. In a healthcare system as vast as the VA, it is a difficult task to ensure that every VA medical center and communitybased outpatient clinic fully implements policies that have been established by VA Office of Mental Health (OMH). An example of a recent policy established by $\mathrm{OMH}$ is that all new veterans contacting a VA facility for treatment services must be scheduled for an initial appointment within 14 days of their contact. Making sure that every facility within the largest health care system in the world consistently implements this 14-day policy is an enormous challenge. Further complicating this challenge is the fact that an unprecedented number of veterans now receive their care from the VA, and more are entering the system every day, putting additional strain on existing resources.

As previously noted, one limitation is that we do not yet know the degree of success of the multiple initiatives launched in recent years. We also do not yet know whether the EBT dissemination and implementation efforts will be effective in terms of reducing the mental health care burden among veterans and active duty personnel. Current efforts are directed towards evaluating the efficacy of the newly developed treatments, and the early findings are very encouraging (Brief, Rubin, Roy, Enggasser, \& Keane, in press; Litz et al., 2007; Morland et al., 2010; Tuerk et al., 2010). In the upcoming years, we expect to have a better understanding of what does and does not work, as well as an understanding of why certain programs and treatments may not have worked. The knowledge gained from these multiple efforts will inform us as we continue in our goal to reduce the mental illness burden among veterans.

Historically, major advances in clinical psychology have been tied to the needs of military service men and women who've been exposed to the traumatic life experiences secondary to service in war zones. The current wars in Iraq and Afghanistan represent another opportunity for clinical psychologists and other mental health care professionals to develop new approaches for lessening the burden of mental illness among our returning military personnel and veterans. Although more work is needed to refine and improve the VA's initial efforts to implement broadly evidence based approaches to assessing and treating psychological conditions, the VA has already taken the critical step of creating bold, contemporary solutions aimed at reducing the burden of mental illness among those that rely on it for care. Further, it demonstrates the VA's commitment to providing exceptional mental health 
services to the many men and women who have served our country and addresses many of the concerns raised by Kazdin and Blase in their seminal review. It is our hope that these advancements will lead to improvements in the mental health and quality of life — not only for veterans, but for all.

\section{Authors' Note}

The opinions expressed in this commentary represent those of the authors and do not represent the Department of Veterans Affairs.

\section{Declaration of Conflicting Interests}

The author declared no potential conflicts of interest with respect to the authorship or the publication of this article.

\section{References}

Brief, D. J., Rubin, A., Roy, M., Enggasser, J., \& Keane, T. M. (in press). Web based interventions for returning veterans with symptoms of posttraumatic stress disorder and risky alcohol use. Journal of Contemporary Psychotherapy.

Cook, J. M., Biyanova, T., \& Coyne, J. C. (2009). Comparative case study of diffusion of eye movement desensitization and reprocessing in two clinical settings: Empirically supported treatment status is not enough. Professional Psychology: Research and Practice, 40, 518-524. doi:10.1037/a0015144

Cook, J. M., Schnurr, P., Biyanova, T., \& Coyne, J. C. (2009). Apples don't fall far from the trees: An Internet survey of influences on psychotherapists' adoption and sustained use of new therapies. Psychiatric Services, 60, 671-676. doi:10.1176/appi.ps.60.5.671

Hoge, C. W., Auchterlonie, J. I., \& Milliken, C. S. (2006). Mental health problems, use of mental health services, and attrition from military service after returning from deployment to Iraq or Afghanistan. Journal of the American Medical Association, 295, 1023-1032. doi:10.1001/jama.295.9.1.1023

Karlin, B. E., Ruzek, J. I., Chard, K. M., Eftekhari, A., Monson, C. M., Hembree, E. A., . . . Foa, E. B. (2010). Dissemination of evidence-based psychological treatments for posttraumatic stress disorder in the Veterans Health Administration. Journal of Traumatic Stress, 23, 663-673. doi:10.1002/jts.20588

Kazdin, A. E. (2008). Evidence-based treatment and practice: New opportunities to bridge clinical research and practice, enhance the knowledge base, and improve patient care. American Psychologist, 63, 146-159. doi:10.1037/0003.066x.63.3.146

Kazdin, A. E., \& Blase, S. L. (2011). Rebooting psychotherapy research and practice to reduce the burden of mental illness.
Perspectives on Psychological Science, 6, 21-37. doi:10.1177/ 1745691610393527

Litz, B., Engel, C., Bryant, R., \& Papa, A. (2007). A randomized, controlled proof-of-concept trial of an Internet-based, therapistassisted self-management treatment for posttraumatic stress disorder. American Journal of Psychiatry, 164, 1676-1684. doi:10.1176/appi.ajp.2007.06122057

Morland, L. A., Greene, C. J., Rosen, C. S., Foy, D., Reilly, P., Shore, J., . . Frueh, B. C. (2010). Telemedicine for anger management therapy in a rural population of combat veterans with posttraumatic stress disorder: A randomized noninferiority trial. Journal of Clinical Psychiatry, 71, 855-863. doi:10.4088/JCP.09m05604blu

Nash, W., Krantz, L., Stein, N., Westphal, R., \& Litz, B. T. (in press). Comprehensive soldier fitness, battlemind, and the stress continuum model: Military organizational approaches to prevention. In J. I. Ruzek, P. P. Schnurr, J. J. Vasterling, \& M. J. Friedman (Eds.), Caring for veterans with deployment-related stress disorders: Iraq, Afghanistan, and beyond. Washington, DC: American Psychological Association.

Pietrzak, R. H., Johnson, D. C., Goldstein, M. B., Malley, J. C., \& Southwick, S. M. (2009). Perceived stigma and barriers to mental healthcare utilization among OEF-OIF veterans. Psychiatric Services, 60, 1118-1122. doi:10.1176/appi.ps.60.8.1118

Rosen, C. S., Chow, H. C., Finney, J. F., Greenbaum, M. A., Moos, R. H., Sheikh, J. I., \& Yesavage, J. A. (2004). VA practice patterns and practice guidelines for treating posttraumatic stress disorder. Journal of Traumatic Stress, 17, 213-222. doi:10.1023/ B:JOTS.0000029264.23878.53

Sloan, D. M., Gallagher, M. W., Feinstein, B. A., Lee, D., \& Pruneau, G. (2011). Efficacy of telehealth treatments for posttraumatic stress-related symptoms: A meta-analysis. Cognitive Behaviour Therapy, 40, 111-125. doi:10.1080/16506073.2010.550058

Smith, A. (2010, August). Home broadband 2010. Washington, DC: Pew Research Center's Internet \& American Life Project. Retrieved from http://pewinternet.org/Reports/2010/HomeBroadband-2010.aspx

Tuerk, P. W., Yoder, M., Ruggiero, K. J., Gros, D. F., \& Acierno, R. (2010). A pilot study of prolonged exposure therapy for posttraumatic stress disorder delivered via telehealth technology. Journal of Traumatic Stress, 23, 116-123. doi:10.1002/jts.20494

Zeiss, A. M., \& Karlin, B. E. (2011). The role of psychology in emerging federal health-care plans. In D. Barlow (Ed.), The Oxford handbook of clinical psychology (pp. 19-183). 183: New York, NY: Oxford University Press. 


\title{
Interventions and Models of Their Delivery to Reduce the Burden of Mental Illness: Reply to Commentaries
}

Perspectives on Psychological Science 6(5) 507-510

(C) The Author(s) 2011

Reprints and permission:

sagepub.com/journalsPermissions.nav DOI: |0.1|77/|74569|6||4|824|

http://pps.sagepub.com

(S)SAGE

\author{
Alan E. Kazdin and Stacey L. Blase \\ Department of Psychology, Yale University, New Haven, CT
}

\begin{abstract}
Our article in the January issue of Perspectives on Psychological Science (Kazdin \& Blase, 20II) recommended developing a portfolio of models to deliver psychotherapeutic interventions with the goals of reaching a larger and more diverse segment of the population in need of mental health services and reducing the burden of mental illness. The commentaries offer several novel extensions to advance the goals. Among the topics raised in the commentaries are the role of moderating influences, the importance of a public health model for intervention research and application, the need to organize and manage our knowledge base and current treatments more effectively, the potential utility of priming-based interventions, the importance of cost measures, and novel applications to extend treatment broadly to veterans in need of services. The commentaries stimulated additional points to address the original goals including the utility of identifying interventions (e.g., lifestyle changes) that can reach many people in need and that can have broad outcome effects on mental and physical health, the importance of "disruptive innovations" (i.e., innovations that qualitatively change the nature of what and how services are delivered) from a business perspective, and the need for improved assessment to track the burden of mental illness in an ongoing way and to evaluate subgroups not being reached with our current interventions.
\end{abstract}

\section{Keywords}

psychological interventions, reducing the burden of mental illness

The personal, social, and monetary burdens of mental illness are enormous. There is a high prevalence rate of psychiatric disorder (25\% in the United States), leaving aside psychosocial dysfunctions that do not meet formal diagnostic criteria but do impair functioning and contribute to the burden of mental illness (Kessler \& Wang, 2008). The majority of individuals who experience dysfunction do not receive services; the paucity of services is particularly acute for several groups (e.g., individuals of a minority or living in rural areas, children, and the elderly). Although advances in developing evidence-based psychotherapies have been remarkable, the dominant model of delivering psychosocial treatment (individual, in-person, one-to-one treatment) is not likely to reach the majority of individuals in need. Our article recommended developing a portfolio of models of delivery with the dual goals of increasing the proportion and diversity of individuals reached with effective interventions and reducing the burden of mental illness (e.g., incidence, prevalence; Kazdin \& Blase, 2011).

We are delighted to have the benefit of such a diverse set of commentators whose contributions to the conceptual, methodological, and empirical literatures on intervention research have been especially influential. We address key issues of the commentaries, and convey how they qualify, alter, and improve on the recommendations in our article. Finally, we conclude with additional points stimulated by the commentaries overall.

\section{Commentaries, Rejoinders, and Perspectives}

Shoham and Insel (2011, this issue) alert us to the importance of searching for moderating influences that may determine which treatment is best for whom. The issue they raise has broad relevance. In virtually all randomized controlled treatment trials (e.g., in oncology, pharmacology, psychotherapy, inter alia) the usual case is that not everyone responds equally well, or at all, to a given treatment-so it is important to

\section{Corresponding Author:}

Alan E. Kazdin, Department of Psychology, 2 Hillhouse Avenue, Yale University, New Haven, CT 06520-8205

E-mail:alan.kazdin@yale.edu 
determine these moderating influences and the best-suited individuals for each treatment. A guiding question for two generations of psychotherapy researchers has focused on the moderators of psychotherapy (e.g., Kiesler, 1971). The most well-cited version of this is "What treatment, by whom is most effective for this individual with that specific problem and under which set of circumstances (Paul, 1967, p. 111). The importance of one of these moderating influences ("for whom") was emphasized by Shoham and Insel in their discussion of Attribute (personal characteristics) $\times$ Intervention effects. We agree greatly with the importance of moderators as reflected in our own research but also in our recreational pastime of trying to figure out what mediated moderation and moderated mediation are, all the while knowing they are important. Yet, in the nature of intervention research and parsimony, one begins by looking for main effects (i.e., treatments that in fact can effect change in most individuals). For example, ethnic and cultural diversity can moderate treatment but we already know that some treatments exert a main effect with diverse groups (Miranda et al., 2005) and getting these to the people can have impact now. Also, the search for moderators is not difficult, but their use for decision making in patient care is not at all straightforward. Among the challenges is establishing high levels of sensitivity and specificity so that patients are assigned to various treatments to which they might be best suited. In addition, many seemingly straightforward moderators including biological and psychological characteristics of the individual and environmental influences have systematic and unsystematic error we are just beginning to understand (e.g., jumping genes, imprinted genetic effects, and epigenetic effects).

Attribute $\times$ Intervention, a first-order interaction, is not likely to capture the "real" interactions that influence outcome. We believe the authors would subscribe to Attribute $x$ Environment $\times$ Intervention effects to recognize the important experience (environment) and attribute (e.g., polymorphisms here and there) combinations. Until there is personalized psychotherapy (á la personalized medicine) that could be scaled up, we need to increase greatly the less personalized, but still effective, psychotherapy. As an analogue, the same amounts of vitamins and minerals are not needed for each individual. We use recommended doses (often based on research) because if those doses reach most people, the health of individuals and our nation would be better. That does not gainsay the benefit for more individualized recommended doses. Effective interventions, whether vitamins or evidence-based psychosocial treatments, need disseminable versions that can be delivered on a large scale.

Shoham and Insel (2011) also remind us that we do not know the mechanisms by which therapeutic change occurs, and this is definitely an important issue, even in current dissemination efforts. An abbreviated intervention may unintentionally sacrifice an important ingredient, as they note. A critical goal for developing a portfolio will be to scale up our interventions for greater reach while maintaining therapeutic impact. Their cogent concern is answered well in the Chorpita et al. (2011, this issue) article in which distillation of common elements of treatment need not necessarily sacrifice effectiveness.

The proposal by Atkins and Frazier (2011, this issue) draws on a successful model from a public health initiative to contain the H1N1 virus. This model integrated three tiers of interventions at the universal, targeted, and intensive levels. This focus on reorganization of existing treatments alerts us to the need for structuring a multilevel approach to mental health, seamlessly integrating and unifying prevention and treatment and using multiple settings (e.g., in the community) and providers (e.g., lay individuals). The three tiers of intervention could address the goal we proposed in our article, namely, reducing the burden of mental illness and unifying disparate intervention models. We concur with their recommendation. Yet, it will still be necessary to scale up the interventions for each of the three tiers. We noted that the intensive intervention level cannot be scaled up now, but their model actually could make the need for intensive treatment less. That is, successful preventive efforts reduce the need for treatment. However, this shifts the need from scaling up intensive treatment to scaling up the universal and targeted interventions. There are many evidence-based interventions at these other two tiers, but those tiers evoke the same tears we shed in relation to treatment. Can we scale them up to reach most people in need? Otherwise, we risk continuing to fail to reach the large portion of the population in need that simply does not have access to these services.

Chorpita et al. highlight the challenge of disseminating our existing treatments and complement the commentary by Atkins and Frazier. The authors contend that if we fail to organize and manage the treatments we currently offer, developing new treatments will not help us to achieve the goal of reducing the burden of mental illness. They highlight the need to strategize better ways to use what we know to make our treatments more easily disseminable. The creative conceptual and empirical work on disseminating common elements of existing treatments could be very important in scaling up our interventions for greater reach. Yet, the challenges remain: Can we scale up effective intervention elements in a form that reaches most people in need? Current common elements that work may still be the individual, one-to-one dominant treatment model, which we argued will need to be complemented by scores of other models.

Shalev and Bargh (2011, this issue) provide a fascinating and novel model for reaching large groups of people in everyday settings. Their suggestion of priming-based interventions is in keeping with our goal of using novel treatment delivery models. Because the nonconscious automatic processes they target are unintentional and operate outside of awareness, priming interventions do not require the volitional engagement of the patient. Thus, interventions can be scaled up to reach many people and do not have to be costly because they can be administered through nontraditional agents and settings. Priming techniques might promote positive emotions, 
evoke self-management or regulation strategies, or foster therapeutic benefits related to such domains as loneliness and isolation. Such interventions might produce change that is therapeutic in its own right or sensitize individuals to change with other interventions that might be easily delivered but less likely to be effective on their own (e.g., communication and public health messages, television appeals). We had argued that reducing the burden of mental illness would profit from, and actually require, collaborations with areas well beyond mental health fields. Shalev and Bargh provide a creative model from social psychology, a sibling field that clearly has much to offer in extending the reach of interventions that can influence mental health.

Yates (2011, this issue) underscores the importance of cost measures of treatment in several ways: cost of delivering the intervention, monetary benefits in outcomes (e.g., patient income from their employment, patient use of health care and social services), and the cost per increment of therapeutic gains. Simply put, cost cannot be ignored in any effort to extend effective interventions so that they reach the many in need of services. This novel measure of "impact per dollar" provides a specific metric that might be useful to integrate in interventions at all levels. Cost is related to the reach of an intervention. Yates notes this aptly by conveying that the challenge is to deliver scaled-up interventions on a plastic spoon (rather than the golden ladle of individual psychotherapy) to as many people as possible.

It is inspiring, instructive, and, for us as citizens, very reassuring to learn of the range of innovations in the Veterans Health Administration (VA), as described by Sloan, Marx, and Keane (2011, this issue). The authors point out the clear intent of this health care system to reach as many people as possible using several delivery opportunities (e.g., Internet, videoconferencing, laypersons, and cell phones). With the portfolio of models already in use, ever-increasing numbers of veterans have access to the services provided by the VA. Naturally, due to its record number of clients, the system experiences the strain of this unprecedented use of its resources. Thus, their example wonderfully illustrates the importance of even further broadening our portfolio of treatment delivery models not only to reach more people in need, but also to sustain their care once they are entered into it. As the largest health care system in the United States, as noted by the authors, with some centralized opportunities and challenges, the VA might be the place to further pursue the goals and the model we suggested. The only additions would be to evaluate empirically the extent of reach (e.g., proportion of individuals in need of services who actually receive them) and the impact on the burden of mental illness (incidence of new disorders or dysfunctions and prevalence). This knowledge might provide key insights that could be transferred outside of the system.

\section{Closing Comments}

A central goal of our article was to focus attention to reducing the burden of mental illness and reaching the large swath of individuals who are not receiving services. Our idea was that a portfolio of models of delivery would be needed to increase the reach of interventions in relation to those in need of services. No one model is likely to reach even a given segment of the population because of the range of real and perceived barriers to seeking or providing services. The commentators have provided novel extensions of our recommendations and have highlighted a multipronged approach that could accelerate advances in reducing the burden. The commentaries have stimulated additional points to address the original goals of our article.

First, we begin the research and services delivery agenda with the goal of reducing the burden of mental illness. We look to how psychotherapy might help and how current knowledge might be used (e.g., common elements of treatment, less costly delivery methods, both in the commentaries), but also we look beyond psychotherapy to examine whether other interventions might contribute. For example, are there non-psychotherapeutic interventions that might address the goal? We have seen primingbased interventions as one possibility in the commentaries. Another would be lifestyle changes that can improve physical and mental health (Walsh, 2011). These changes include exercise, better nutrition and diet, time in nature, improved relationships, recreation and enjoyable activities relaxation and stress management, spiritual involvement, and service to others, several of which have an evidence base already. One or more of these lifestyle changes may be feasible as a type of intervention that can serve both to prevent and treat psychological impairment and dysfunction. An added strength is that some of the same lifestyle interventions promote both physical and mental health. This adds a different dimension to our original article, namely that, when possible, high priority might be given to interventions that have reach within the population in need but that also produce broad or cascading therapeutic effects beyond some target focus (e.g., depression, anxiety). Such interventions would be very sensitive to the cost issues also raised in the commentaries.

Second, we see diverse disciplines as relevant to reducing the burden of mental illness, even if all of the interventions were psychotherapy in one form or another. Other disciplines have specialties that will help with penetration of our interventions to potential consumers. As one example, the notion of disruptive technology or disruptive innovation in business refers to innovations that alter a product and its delivery in novel ways. The change or innovation is not the usual evolutionary or incremental step in product development, but rather it provides something different and serves-indeed develops - a market that is not being served (e.g., Christensen, Grossman, \& Hwang, 2009). Examples are evident in manufacturing (e.g., interchangeable parts, assembly line in car production), new products (e.g., cell phone, smartphone, tablet), consumer purchasing (e.g., credit cards, apps to make purchases with smartphones), and health care (e.g., home pregnancy tests, services such as flu shots or blood pressure testing in stores and shopping malls). Such interventions often 
provide simpler, less expensive, or more convenient solutions to problems and can be scaled to reach people. Packaging our interventions so some of them are "disruptive" could have huge impact for the goal of reducing the burden of mental illness.

Finally, and perhaps most central to the goal, is the need for improved assessment along two fronts. First, ongoing (regular) assessment at the national level will be needed to measure the burden of mental illness. We mentioned in our article models already available that could be brought to bear. We will need assessment of indices of the burden of mental illness to provide a backdrop and baseline for evaluating progress. The assessment of indices of the burden of mental illness might include such measures as incidence, prevalence, impairment, or disability-adjusted life years (disease burden), or quality of life years.

Second, assessment will be needed to evaluate what subgroups are and are not being reached with the portfolio of evidence-based interventions. The portfolio of delivery models is not a list of more creative ways to reach the same people or to modernize the dominant model of delivery (e.g., use of technology but still delivering one-to-one treatment). Multiple parties are in need of care, and we would benefit from seeing who was and was not effectively reached by our overlapping models of treatment delivery. That same assessment might well guide the development of treatments or turn the turrets of interventions in one area to a target group in need of services but not yet reached.

A portfolio of models of delivery was designed to reach diverse segments of the population in need. To illustrate our purpose in doing so, we borrow from an example in the field of visual arts. In a Jackson Pollock painting, different quantities of different colors of overlapping paint are applied through a variety of creative methods to cover an entire canvas. Such methods of paint application included the use of brushes, sticks, trowels, knives, basting syringes, and flinging or directly pouring or dripping the paint onto the canvas, among other nontraditional means - all with a purportedly intentional vision of how the piece should appear when complete (Pollock, 1947-1948). As applied to our portfolio idea, we need different but overlapping intervention models (e.g., our paint) applied through a variety of creative deliveries (e.g., our brushes, sticks, trowels) to reach as many people as possible (e.g., to cover our canvas of people in need). We need overlapping models because no single delivery model is likely to be perfectly suited to a given population or subpopulation. Again, the most critical point of departure for progress may be beginning to reduce the burden of mental illness, obtaining measures that will allow us to evaluate progress, and then developing models of delivery that improve in their reach and scalability. We are grateful to the commentators for elaborating in creative ways the range of options that might be used to accomplish these goals.

\section{Declaration of Conflicting Interests}

The author(s) declared no potential conflicts of interest with respect to the research, authorship, and/or publication of this article.

\section{Funding}

The author(s) received no financial support for the research, authorship, and/or publication of this article.

\section{References}

Atkins, M., \& Frazier, S. (2011). Expanding the toolkit or changing the paradigm: Are we ready for a public health approach to mental health? Perspectives on Psychological Science, 6, 483-487.

Chorpita, B., Rotheram-Borus, M. J., Daleiden, E., Bernstein, A., Cromley, T., Swendeman, D., \& Regan, J. (2011). The old solutions are the new problem: How do we better use what we already know about reducing the burden of mental illness? Perspectives on Psychological Science, 6, 493-497.

Christensen, C. M., Grossman, J. H., \& Hwang, J. (2009). The innovator's prescription: A disruptive solution for health care. New York, NY: McGraw-Hill.

Kazdin, A. E., \& Blase, S. L. (2011). Rebooting psychotherapy research and practice to reduce the burden of mental illness. Perspectives on Psychological Science, 6, 21-37.

Kessler, R. C., \& Wang, P. S. (2008). The descriptive epidemiology of commonly occurring mental disorders in the United States. Annual Review of Public Health, 29, 115-129.

Kiesler, D. J. (1971). Experimental designs in psychotherapy research. In A. E. Bergin \& S. L. Garfield (Eds.), Handbook of psychotherapy and behavior change: An empirical analysis (pp. 36-74). New York, NY: Wiley.

Miranda, J., Bernal, G., Lau, A. S., Kohn, L., Hwang, W. C., \& LaFromboise, T. (2005). State of the science on psychosocial interventions for ethnic minorities. Annual Review of Clinical Psychology, 1, 113-143.

Paul, G. L. (1967). Outcome research in psychotherapy. Journal of Consulting Psychology, 31, 109-118.

Pollock, J. (1947-1948). My painting. Possibilites, 1, 78-83.

Shalev, I., \& Bargh, J. (2011). Use of priming-based interventions to facilitate psychological health: Commentary on Kazdin \& Blase (2011). Perspectives on Psychological Science, 6, 488-492.

Shoham, V., \& Insel, T. R. (2011). Rebooting for whom? Portfolios, technology, and personalized intervention. Perspectives on Psychological Science, 6, 478-482.

Sloan, D. M., Marks, B. P., \& Keane, T. M. (2011). Reducing the burden of mental illness in military veterans: Commentary on Kazdin \& Blase (2011). Perspectives on Psychological Science, 6, 503-506.

Walsh, R. (2011). Lifestyle and mental health. American Psychologist. Advance online publication. doi:10.1037/a0021769

Yates, B. (2011). Delivery systems can determine therapy costs and effectiveness, more than type of therapy. Perspectives on Psychological Science, 6, 498-502. 